\title{
Pd(II)-Catalyzed Synthesis of Alkylidene Phthalides via Decarbonylative Annulation Reaction
}

\author{
Somadrita Borthakur, ${ }^{\dagger}$ Swagata Baruah, ${ }^{\dagger}$ Bipul Sarma, ${ }^{\dagger}$ and Sanjib Gogoi $*, \dagger$ \\ $\dagger$ Applied Organic Chemistry, Chemical Sciences \& Technology Division, CSIR-North East \\ Institute of Science and Technology, Jorhat-785006, AcSIR, India, and $\ddagger$ Department of \\ Chemical Sciences, Tezpur University, Tezpur-784028 India, Fax: +913762370011 Tel.: +91 \\ 3762372948; skgogoi1@gmail.com; sanjibgogoi@neist.res.in
}

\section{Supporting Information}




\section{General Information}

Melting points were measured with a Buchi B-540 melting point apparatus and are uncorrected. NMR spectra were recorded on Bruker Avance III $500 \mathrm{MHz}$ FTNMR spectrometer using tetramethylsilane (TMS) as an internal standard. All the commercially available regents were used as received. All experiments were monitored by thin layer chromatography. TLC was performed on Merck TLC Silica gel $60 \mathrm{~F}_{254}$ precoated plates. Column chromatography was performed on silica gel (100-200 mesh, Merck).

\section{Reaction Procedures}

\subsection{General procedure for the synthesis of 3-arylnaphthaquinone derivatives (1)}

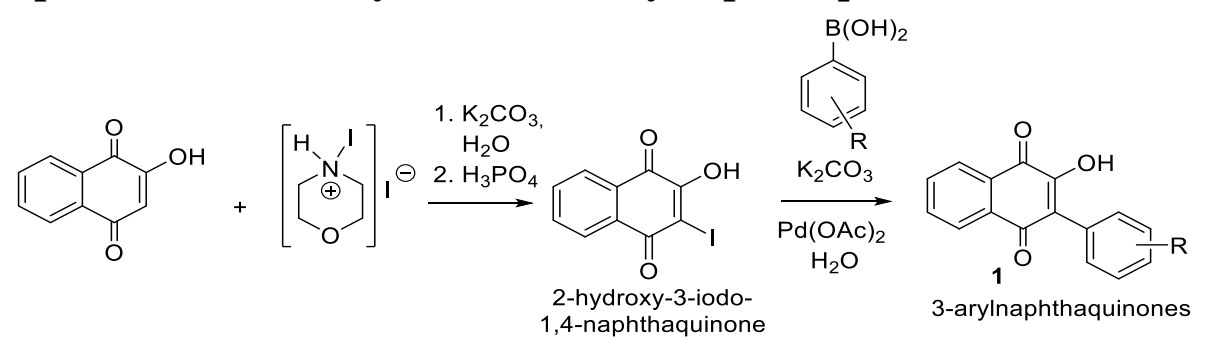

Scheme SI-1

Step 1: Preparation of 2-hydroxy-3-iodo-1,4-naphthaquinone: To a stirred solution of 2hydroxy-1,4-naphthaquinone $(5.22 \mathrm{~g}, 30 \mathrm{mmol})$ in water $(200 \mathrm{~mL})$, morpholine-iodine complex $(12.77 \mathrm{~g}, 37.5 \mathrm{mmol})$ and $\mathrm{K}_{2} \mathrm{CO}_{3}(12.42 \mathrm{~g}, 90 \mathrm{mmol})$, were added in small portion wise manner over a period of 1 hour. The reaction mixture was then stirred for 1 hour. Subsequently, the mixture was cooled using an ice bath and acidified with concentrated $\mathrm{H}_{3} \mathrm{PO}_{4}$. The solution was left to react under agitation at room temperature for 24 hours after which the mixture was filtered and the solid obtained was washed with ice-cold water. The solid was dried to get the desired product.

Step 2: Preparation of 3-aryl-2-hydroxy naphthaquinones (1): The mixture of 2-hydroxy-3iodo-1,4-naphthaquinone (300 mg, $1.0 \mathrm{mmol}), \mathrm{K}_{2} \mathrm{CO}_{3}(690 \mathrm{mg}, 5.0 \mathrm{mmol}$ ), phenylboronic acid $(183 \mathrm{mg}, 1.5 \mathrm{mmol})$ and $\mathrm{Pd}(\mathrm{OAc})_{2}(5 \mathrm{~mol} \%)$ was refluxed in $10 \mathrm{~mL}$ water for 6 hours. The reaction mixture was then acidified with concentrated $\mathrm{H}_{3} \mathrm{PO}_{4}$. The solid that formed was vacuum filtered and purified by column chromatography to get the desired product. 


\subsection{General procedure for the synthesis of 3-(1H-inden-1- ylidene)isobenzofuran-1(3H)-one 3:}

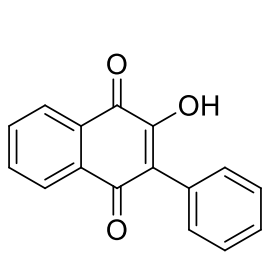

1

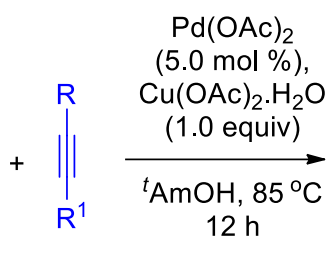

2

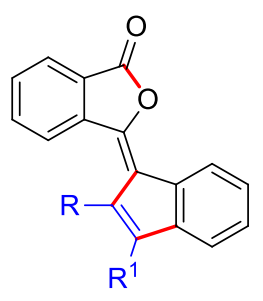

3

\section{Scheme SI-2}

A mixture of 3-phenyl-2-hydroxy-1,4-naphthaquinone (1, $0.2 \mathrm{mmol})$, alkyne (2, $0.2 \mathrm{mmol})$, $\mathrm{Pd}(\mathrm{OAc})_{2}(5.0 \mathrm{~mol} \%)$ and $\mathrm{Cu}(\mathrm{OAc})_{2} \cdot \mathrm{H}_{2} \mathrm{O}\left(1.0\right.$ equiv) in ${ }^{t} \mathrm{AmOH}(5.0 \mathrm{~mL})$ was stirred at $85{ }^{\circ} \mathrm{C}$ under open air for 12 hours. The solvent was removed under vacuo and the crude product thus obtained was purified by silica gel (100-200 mesh) column chromatography using EtOAc/Hexane as the eluant to afford 3.

\section{Mechanistic Experiments}

\subsection{Intermolecular competition experiments:}

(a) A solution of 3-phenyl-2-hydroxy-1,4-naphthaquionone $1 \mathbf{a}$ (50 mg, $0.2 \mathrm{mmol}$ ), alkyne $\mathbf{2 b}$ (41 $\mathrm{mg}, 0.2 \mathrm{mmol})$, alkyne $2 \mathrm{~d}$ (43 mg, $0.2 \mathrm{mmol}), \mathrm{Pd}(\mathrm{OAc})_{2}(2 \mathrm{mg}, 5.0 \mathrm{~mol} \%)$ and $\mathrm{Cu}(\mathrm{OAc})_{2} \cdot \mathrm{H}_{2} \mathrm{O}$ (40 mg, $0.2 \mathrm{mmol})$ in ${ }^{t} \mathrm{AmOH}(5 \mathrm{~mL})$ was stirred at $85^{\circ} \mathrm{C}$ under open air for 12 hours.

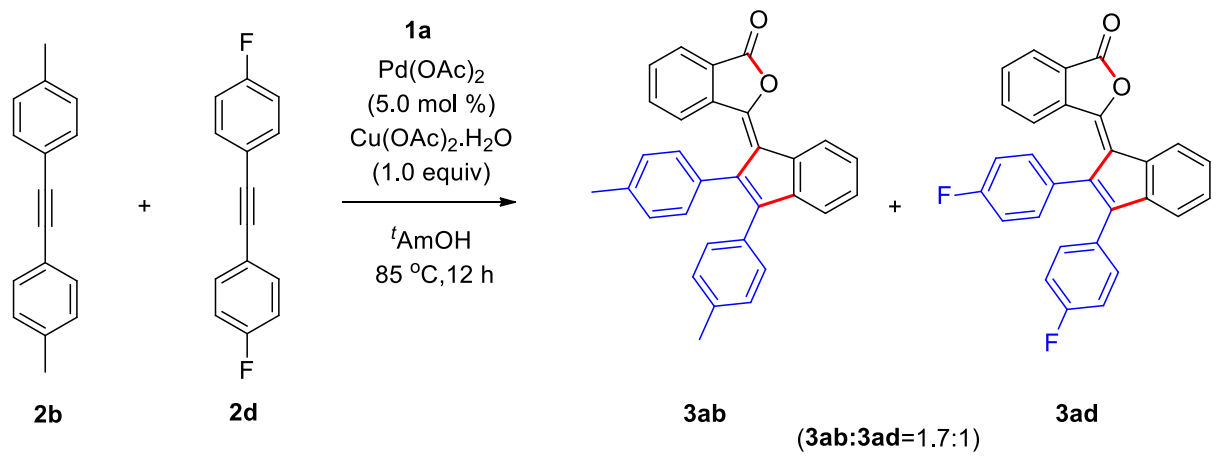

\section{Scheme SI-3}

The crude product obtained was purified by silica gel (100-200 mesh) column chromatography using EtOAc/Hexane (1:9) as the eluant to afford a mixture of products 3ab and 3ad in the ratio 3ab:3ad $=1.7: 1$. 
(b) A solution of hydroxynaphthaquinone $1 \mathbf{b}(53 \mathrm{mg}, 0.2 \mathrm{mmol})$, hydroxynaphthaquinone $1 \mathbf{f}$ (57 $\mathrm{mg}, 0.2 \mathrm{mmol})$, alkyne $2 \mathrm{a}(36 \mathrm{mg}, 0.2 \mathrm{mmol}), \mathrm{Pd}(\mathrm{OAc})_{2}(2 \mathrm{mg}, 5.0 \mathrm{~mol} \%)$ and $\mathrm{Cu}(\mathrm{OAc})_{2} \cdot \mathrm{H}_{2} \mathrm{O}$ (40 mg, $0.2 \mathrm{mmol})$ in ${ }^{t} \mathrm{AmOH}(5 \mathrm{~mL})$ was stirred at $85^{\circ} \mathrm{C}$ under open air for 12 hours.

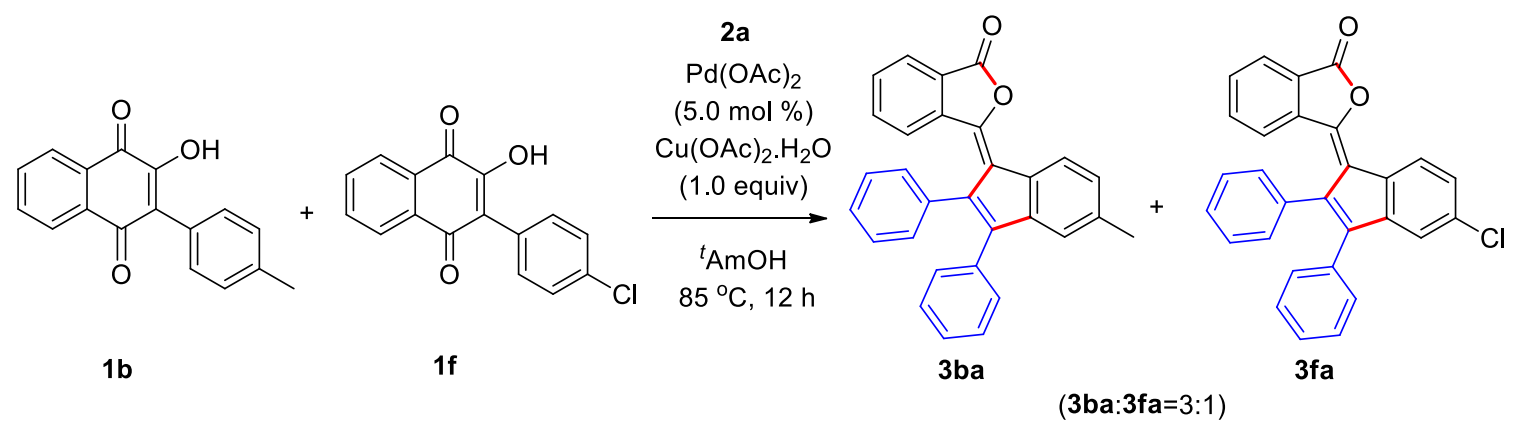

\section{Scheme SI-4}

The crude product obtained was purified by silica gel (100-200 mesh) column chromatography using EtOAc/Hexane (1:9) as the eluant to afford a mixture of products $\mathbf{3 b a}$ and $\mathbf{3 f a}$ in the ratio 3ba:3fa $=3: 1$.

\subsection{Isotopically labelled experiments}

(a) A solution of hydroxynaphthaquinone 1a $(50 \mathrm{mg}, 0.2 \mathrm{mmol}), \mathrm{Pd}(\mathrm{OAc})_{2}(2 \mathrm{mg}, 5.0 \mathrm{~mol} \%)$ and $\mathrm{Cu}(\mathrm{OAc})_{2} \mathrm{H}_{2} \mathrm{O}(40 \mathrm{mg}, 0.2 \mathrm{mmol})$ in $\mathrm{CD}_{3} \mathrm{OD}(3.0 \mathrm{~mL})$ was stirred at $64{ }^{\circ} \mathrm{C}$ under argon for 12 hours. The solvent was evaporated and the crude product obtained was purified by silica gel (100-200 mesh) column chromatography using EtOAc/Hexane (1:9) as the eluant. The H/D exchange was found to be $0 \%$ at the protons attached to C-2' and C- 6 ' in the recovered hydroxyl naphthaquinone 1a-D.

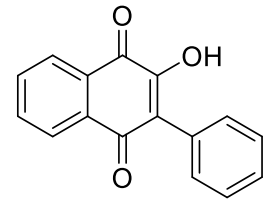

1 a

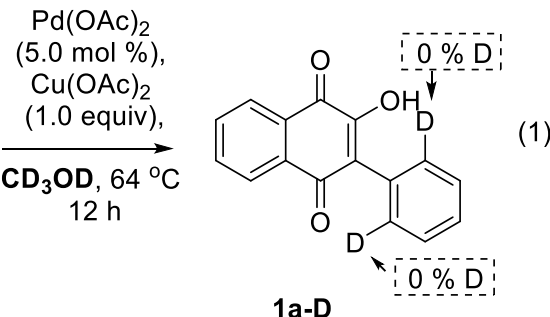

1a-D

\section{Scheme SI-5}

(b) Synthesis of 1a-D5: The synthetic route for 1a-D5 is shown in scheme SI-6.

(i) Synthesis of bromobenzene-D5: To a stirred mixture of sulfuric acid $(9 \mathrm{~mL})$ in water (33 $\mathrm{mL})$, benzene $\mathrm{D}_{6}(4.2 \mathrm{~g}, 50 \mathrm{mmol})$ was added very slowly at $0{ }^{\circ} \mathrm{C}$. Then, $\mathrm{NaBrO}_{3}(55 \mathrm{mmol})$ was added into the reaction mixture at the same temperature in two portions with an interval of one 
hour. The reaction was continued for another 10 hours at normal temperature. Then, it was poured in ice water, extracted with diethyl ether $\left(100 \mathrm{~mL}\right.$ x 3) and dried over $\mathrm{Na}_{2} \mathrm{SO}_{4}$. The solvent was removed under vacuo and the crude product obtained was used for the next step without purification $(6.9 \mathrm{~g}, 86 \%)$.

(ii) Synthesis of (Ds-phenyl)boronic acid: A round bottom flask fitted with magnetic stirring bar was charged with bromobenene- $\mathrm{d}_{5}(2.0 \mathrm{~g}, 12.3 \mathrm{mmol})$ under nitrogen atmosphere. Then, anhydrous THF $(20 \mathrm{~mL})$ was added and the solution was cooled to $-78{ }^{\circ} \mathrm{C}$. To this solution, $n$ butyllithium $(5.9 \mathrm{~mL}, 2.5 \mathrm{M}, 14.76 \mathrm{mmol})$ was added in dropwise fashion over 30 minutes. The solution was stirred at $-78{ }^{\circ} \mathrm{C}$ for 2 hours and then triisopropyl borate $(4.3 \mathrm{~mL}, 18.45 \mathrm{mmol})$ was added drop wise into the reaction mixture. The solution was allowed to warm to room temperature overnight. After that the reaction was quenched with dilute $\mathrm{HCl}(20 \%, 20 \mathrm{~mL})$ and the reaction mixture was stirred for 3 hours at room temperature. The resulted biphasic solution was extracted with ethyl acetate $(50 \mathrm{~mL} \times 2)$. The organic layer was washed twice with water, dried over sodium sulphate and concentrated using rotary evaporation. To the crude product, hexane $(22 \mathrm{~mL})$ was added. The white ( $\mathrm{d}_{5}$-phenyl)boronic acid solid precipitated out from hexane was filtered, dried and used without further purification (77\%).

(iii) Synthesis of 3-aryl-2-hydroxy naphthaquinone-ds: A mixture of 2-hydroxy-3-iodo-1,4naphthaquinone (300 mg, $1.0 \mathrm{mmol}$ ), $\mathrm{K}_{2} \mathrm{CO}_{3}(690 \mathrm{mg}, 5.0 \mathrm{mmol}$ ), (d5-phenyl)boronic acid (191 $\mathrm{mg}, 1.5 \mathrm{mmol})$ and $\mathrm{Pd}(\mathrm{OAc})_{2}(5 \mathrm{~mol} \%)$ was refluxed in $10 \mathrm{~mL}$ water for 6 hours. The reaction mixture was then acidified with concentrated $\mathrm{H}_{3} \mathrm{PO}_{4}$. The solid that formed was vacuum filtered and purified by column chromatography to get the desired product. ${ }^{1} \mathrm{H}$ NMR $\left(500 \mathrm{MHz}, \mathrm{CDCl}_{3}\right.$ ) $\delta 8.22(\mathrm{~d}, J=7.5 \mathrm{~Hz}, 1 \mathrm{H}), 7.47(\mathrm{~d}, J=7.5 \mathrm{~Hz}, 1 \mathrm{H}), 7.82(\mathrm{t}, J=7.0 \mathrm{~Hz}, 1 \mathrm{H}), 7.79(\mathrm{t}, J=7.0 \mathrm{~Hz}$, $1 \mathrm{H})$.

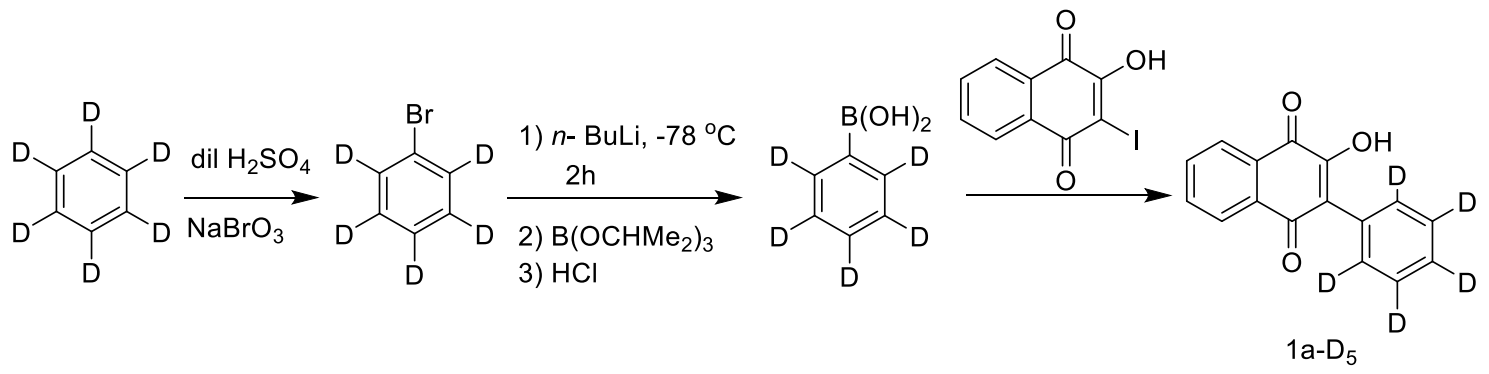

Scheme SI-6 
(c) Intermolecular competitive experiment between substrates 1a and 1a-D5:

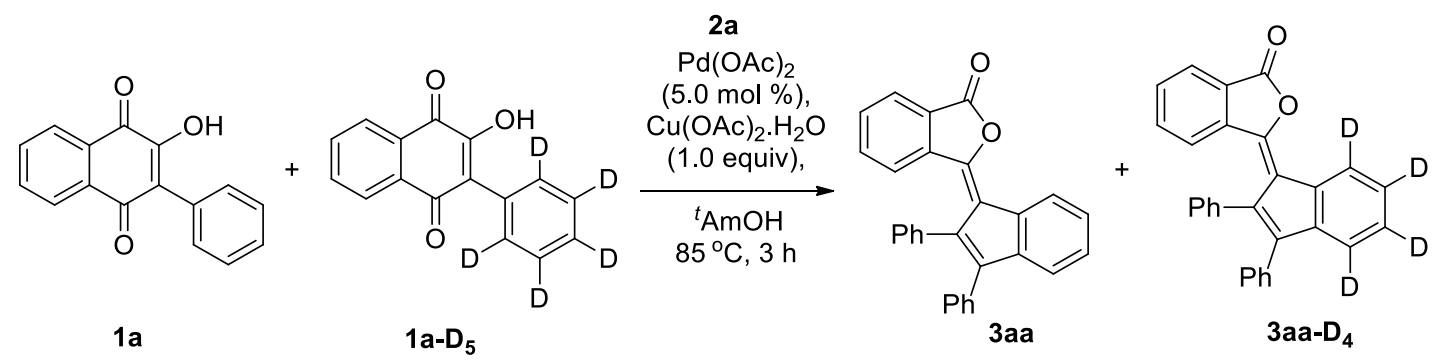

Scheme SI-7

A mixture of hydroxynaphthaquinones 1a $(125 \mathrm{mg}, 0.5 \mathrm{mmol})$ and 1a-D5 (128 $\mathrm{mg}, 0.5 \mathrm{mmol})$, alkyne $2 \mathbf{a}(89 \mathrm{mg}, 0.5 \mathrm{mmol}), \mathrm{Pd}(\mathrm{OAc})_{2}(6 \mathrm{mg}, 5.0 \mathrm{~mol} \%)$ and $\mathrm{Cu}(\mathrm{OAc})_{2} \mathrm{H}_{2} \mathrm{O}(100 \mathrm{mg}, 0.5$ $\mathrm{mmol})$ in ${ }^{t} \mathrm{AmOH}(5.0 \mathrm{~mL})$ was stirred at $85{ }^{\circ} \mathrm{C}$ under open air for 3 hours. The solvent was removed under vacuo and the crude product thus obtained was purified by silica gel (100-200 mesh) column chromatography using EtOAc/Hexane (1:9) as the eluant to provide a mixture of 3aa and 3aa-D4. The ratio of 3aa and 3aa-D4 was determined to be 2.6:1 by ${ }^{1} \mathrm{H}$ NMR integration $\operatorname{method}\left(k_{\mathrm{H}} / k_{\mathrm{D}}=2.6\right)$.

(d) Competitive parallel experiment between substrates 1a and 1a-D5:

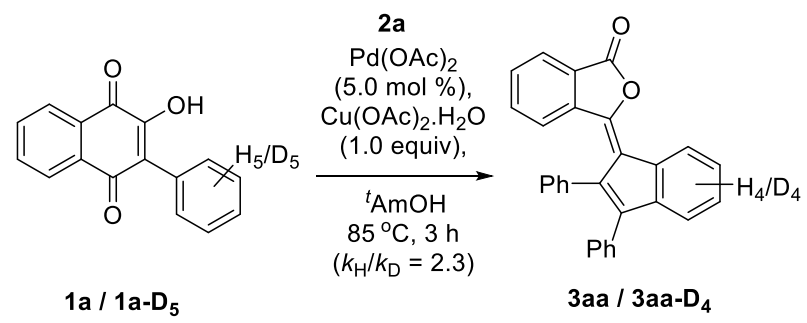

\section{Scheme SI- 8}

A solution of hydroxynaphthaquionone 1a $(50 \mathrm{mg}, 0.2 \mathrm{mmol})$, alkyne $\mathbf{2 a}(36 \mathrm{mg}, 0.2 \mathrm{mmol})$, $\mathrm{Pd}(\mathrm{OAc})_{2}(2 \mathrm{mg}, 5.0 \mathrm{~mol} \%)$ and $\mathrm{Cu}(\mathrm{OAc})_{2} \mathrm{H}_{2} \mathrm{O}(40 \mathrm{mg}, 0.2 \mathrm{mmol})$ in ${ }^{t} \mathrm{AmOH}(4 \mathrm{~mL})$ was stirred at $85{ }^{\circ} \mathrm{C}$ under open air for 3 hours. The solvent was evaporated and the crude product obtained was purified carefully by silica gel (100-200 mesh) column chromatography using EtOAc/Hexane (1:9) as the eluant. Parallely, in another round bottom flask, a solution of hydroxynaphthaquionone 1a-D5 (51 mg, $0.2 \mathrm{mmol})$, alkyne $\mathbf{2 a}(36 \mathrm{mg}, 0.2 \mathrm{mmol}), \mathrm{Pd}(\mathrm{OAc})_{2}(2$ $\mathrm{mg}, 5.0 \mathrm{~mol} \%)$ and $\mathrm{Cu}(\mathrm{OAc})_{2} \mathrm{H}_{2} \mathrm{O}(40 \mathrm{mg}, 0.2 \mathrm{mmol})$ in ${ }^{t} \mathrm{AmOH}(5 \mathrm{~mL})$ was stirred at $85^{\circ} \mathrm{C}$ under open air for 3 hours. The solvent was evaporated and the crude product obtained was 
purified carefully by silica gel (100-200 mesh) column chromatography using EtOAc/Hexane $(1: 9)$ as the eluant. The ratio of 3aa and 3aa-D 4 was determined to be 2.3:1 $\left(k_{\mathrm{H}} / k_{\mathrm{D}}=2.3\right)$.

4. Test for evolution of $\mathrm{CO}$ gas: $\mathrm{PdCl}_{2}(300 \mathrm{mg})$ was dissolved in two drops of conc. $\mathrm{HCl}$ and diluted with $15 \mathrm{~mL}$ of distilled water. A cold saturated solution of phosphomolybdic acid in water was prepared separately. These two solutions were then mixed in a separate vial in 1:2 ( $\mathrm{PMA}: \mathrm{PdCl}_{2}$ ) ratio. Some narrow pieces of filter papers were then dipped in this $\mathrm{PMA}-\mathrm{PdCl}_{2}$ solution and then these were dried at room temperature for 1 hour. In a round bottom flask, 3phenyl-2-hydroxy-1,4-naphthaquionone (1, $0.4 \mathrm{mmol})$, alkyne (2, $0.4 \mathrm{mmol}), \mathrm{Pd}(\mathrm{OAc})_{2}(5.0 \mathrm{~mol}$ $\%$ ) and $\mathrm{Cu}(\mathrm{OAc})_{2} \cdot \mathrm{H}_{2} \mathrm{O}$ (1.0 equiv) were taken and $8.0 \mathrm{~mL}$ of ${ }^{t} \mathrm{AmOH}$ was added into this reaction mixture. Then, one strip of the above dried filter paper was fitted inside the round bottom flask with the help of a septum as shown in the pictures below. The reaction mixture was then heated at $85{ }^{\circ} \mathrm{C}$. After two hours of heating, it was observed that the yellow colour of the strip was changed to dark-blue color, indicating the evolution of $\mathrm{CO}$ gas from the reaction mixture. ${ }^{1}$

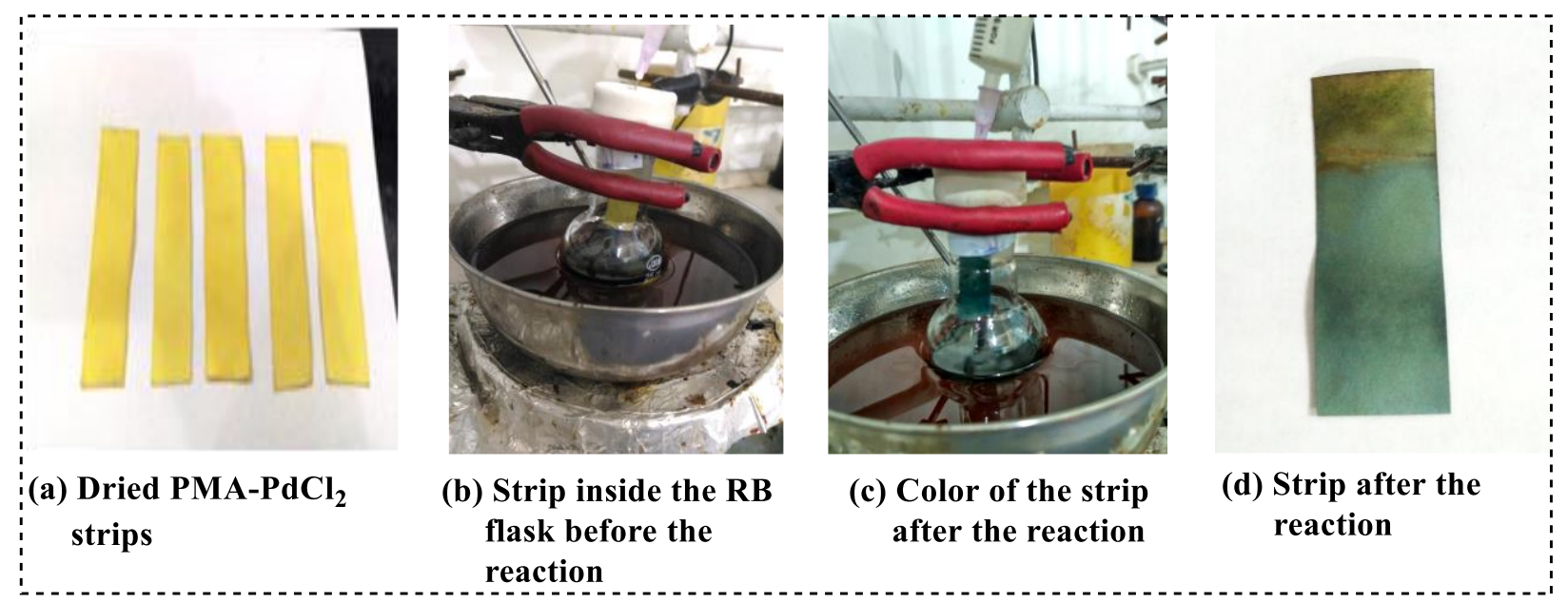

Figure SI-1. Test for CO elimination 


\section{Spectral and Analytical Data:}
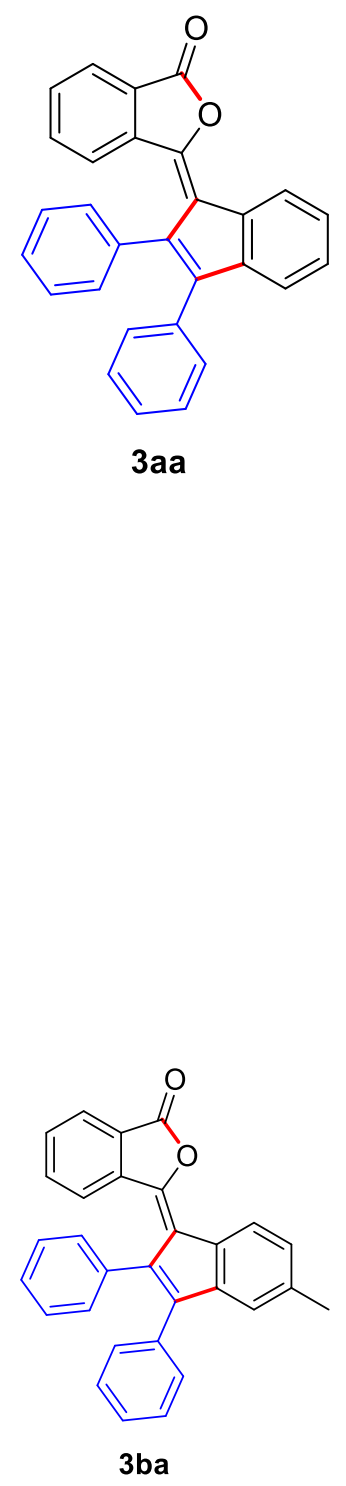

\section{(E)-3-(2,3-Diphenyl-1 $H$-inden-1-ylidene)isobenzofuran-1(3H)-one}

(3aa): A mixture of 3-phenyl-2-hydroxy-1,4-naphthaquinone (1a, $250 \mathrm{mg}, 1.0$ mmol), alkyne (2a, $180 \mathrm{mg}, 1.0 \mathrm{mmol}), \mathrm{Pd}(\mathrm{OAc})_{2}(10 \mathrm{mg}, 5.0 \mathrm{~mol} \%)$ and $\mathrm{Cu}(\mathrm{OAc})_{2} \cdot \mathrm{H}_{2} \mathrm{O}(200 \mathrm{mg} .1 .0 \mathrm{mmol})$ in ${ }^{t} \mathrm{AmOH}(8.0 \mathrm{~mL})$ was stirred at $85{ }^{\circ} \mathrm{C}$ under open air for 12 hours. The solvent was removed under vacuo and the crude product thus obtained was purified by silica gel (100-200 mesh) column chromatography using EtOAc/Hexane (1:9) as the eluant to afford 3aa. The title compound was obtained as dark orange solid with $350 \mathrm{mg}(88 \%)$ yield. M.p.: $225-228{ }^{\circ} \mathrm{C} .{ }^{1} \mathrm{H}$ NMR $\left(500 \mathrm{MHz} \mathrm{CDCl}_{3}\right) \delta 8.57$ (d, $J=7.7$ $\mathrm{Hz}, 1 \mathrm{H}), 7.93(\mathrm{~d}, J=7.7 \mathrm{~Hz}, 1 \mathrm{H}), 7.41$ (t, $J=7.5 \mathrm{~Hz}, 1 \mathrm{H}), 7.39-7.23$ (m, $13 \mathrm{H}), 7.12$ (t, $J=8.4 \mathrm{~Hz}, 1 \mathrm{H}), 5.72(\mathrm{~d}, J=8.2 \mathrm{~Hz}, 1 \mathrm{H}) .{ }^{13} \mathrm{C}$ NMR (125 $\left.\mathrm{MHz} \mathrm{CDCl}_{3}\right) \delta 166.2,147.8,145.6,142.0,137.7,136.7,136.0,134.8$, $134.3,133.7,130.8,130.3,129.5,129.1,128.2,128.1,127.7,127.5$, 127.3, 126.9, 126.8, 126.1, 125.9, 125.4, 120.8. HRMS (+ESI) Calcd for $\mathrm{C}_{29} \mathrm{H}_{19} \mathrm{O}_{2}[\mathrm{M}+\mathrm{H}]^{+}:$399.1385; found: 399.1380 .

\section{(E)-3-(5-Methyl-2,3-diphenyl-1H-inden-1-ylidene)isobenzofuran-}

1(3H)-one (3ba): The title compound was prepared by following the general procedure 2.2 and obtained as dark orange solid with $70 \mathrm{mg}$ (85\%) yield. M.p.: $218{ }^{\circ} \mathrm{C} .{ }^{1} \mathrm{H}$ NMR $\left(500 \mathrm{MHz}, \mathrm{CDCl}_{3}\right) \delta 8.43(\mathrm{~d}, J=$ $7.9 \mathrm{~Hz}, 1 \mathrm{H}), 7.92$ (d, $J=7.6 \mathrm{~Hz}, 1 \mathrm{H}), 7.39$ (t, $J=7.4 \mathrm{~Hz}, 1 \mathrm{H}), 7.35-7.33$ (m, 2H), $7.32(\mathrm{~d}, J=0.7 \mathrm{~Hz}, 1 \mathrm{H}), 7.31$ (d, $J=1.8 \mathrm{~Hz}, 1 \mathrm{H}), 7.31-7.30$ (m, 1H), 7.29-7.23 (m, 5H), 7.18-7.16 (m, 1H), 7.12-7.09 (m, 2H), $5.71(\mathrm{~d}$, $J=8.2 \mathrm{~Hz}, 1 \mathrm{H}), 2.40(\mathrm{~s}, 3 \mathrm{H}) .{ }^{13} \mathrm{C} \mathrm{NMR}\left(125 \mathrm{MHz}, \mathrm{CDCl}_{3}\right) \delta 166.3$, $147.2,145.7,142.3,138.5,137.8,136.8,135.1,134.4,133.6,133.4$, $130.7,130.0,129.5,129.0,128.1,127.6,127.4,127.1,126.7,126.2$, 125.8, 125.3, 121.6, 21.7. HRMS (+ESI) Calcd for $\mathrm{C}_{30} \mathrm{H}_{21} \mathrm{O}_{2}[\mathrm{M}+\mathrm{H}]^{+}$: 413.1542; found: 413.1538 . 


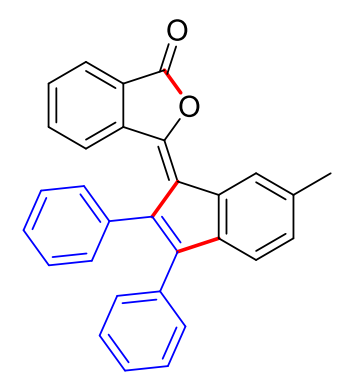

$3 \mathrm{ca}$

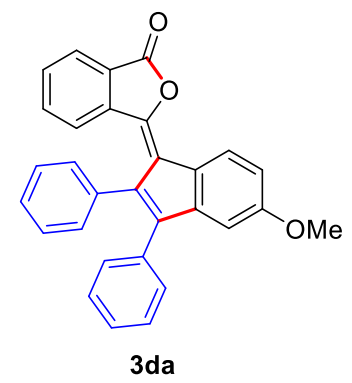

(E)-3-(6-Methyl-2,3-diphenyl-1H-inden-1-ylidene)isobenzofuran-

1(3H)-one (3ca): The title compound was prepared by following the general procedure 2.2 and obtained as dark orange solid with $64 \mathrm{mg}$ (77\%) yield. M.p.: 206-208 ${ }^{\circ} \mathrm{C} .{ }^{1} \mathrm{H}$ NMR $\left(500 \mathrm{MHz}, \mathrm{CDCl}_{3}\right) \delta 8.39$ (s, 1H), 7.93 (d, $J=7.7 \mathrm{~Hz}, 1 \mathrm{H}), 7.41$ (t, $J=7.5 \mathrm{~Hz}, 1 \mathrm{H}), 7.36-7.09$ (m, $13 \mathrm{H}), 5.74(\mathrm{~d}, J=8.2 \mathrm{~Hz}, 1 \mathrm{H}), 2.52(\mathrm{~s}, 3 \mathrm{H}) .{ }^{13} \mathrm{C} \mathrm{NMR}(125 \mathrm{MHz}$, $\left.\mathrm{CDCl}_{3}\right) \delta 166.4,147.4,145.7,139.6,137.8,136.8,136.4,134.5,134.0$, $133.6,130.8,130.1,129.5,129.0,128.8,128.2,128.1,127.5,127.4$, 126.8, 125.8, 125.3, 120.5, 21.0. HRMS (+ESI) Calcd for $\mathrm{C}_{30} \mathrm{H}_{21} \mathrm{O}_{2}$ $[\mathrm{M}+\mathrm{H}]^{+}:$413.1542; found: 413.1544 .

\section{(E)-3-(5-Methoxy-2,3-diphenyl-1H-inden-1-ylidene)isobenzofuran-}

1(3H)-one (3da): The title compound was prepared by following the general procedure 2.2 and obtained as dark orange solid with $68 \mathrm{mg}$ (80\%) yield. M.p.: $241-244{ }^{\circ} \mathrm{C} .{ }^{1} \mathrm{H}$ NMR $\left(500 \mathrm{MHz}, \mathrm{CDCl}_{3}\right) \delta 8.46$ (d, $J$ $=8.8 \mathrm{~Hz}, 1 \mathrm{H}), 7.90(\mathrm{~d}, J=7.6 \mathrm{~Hz}, 1 \mathrm{H}), 7.38(\mathrm{t}, J=7.5 \mathrm{~Hz}, 1 \mathrm{H}), 7.36-$ 7.29 (m, 6H), 7.29-7.25 (m, 2H), $7.22(\mathrm{dd}, J=7.9,1.8 \mathrm{~Hz}, 2 \mathrm{H}), 7.10$ (dd, $J=8.4,3.0 \mathrm{~Hz}, 1 \mathrm{H}), 6.86(\mathrm{~d}, J=8.2 \mathrm{~Hz}, 2 \mathrm{H}), 5.66(\mathrm{~d}, J=8.2 \mathrm{~Hz}, 1 \mathrm{H})$, $3.86(\mathrm{~s}, 3 \mathrm{H}) .{ }^{13} \mathrm{C} \mathrm{NMR}\left(125 \mathrm{MHz}, \mathrm{CDCl}_{3}\right) \delta 166.4,160.3,146.6,145.1$, $144.0,137.8,136.7,136.2,134.2$, 133.6, 130.7, 129.9, 129.5, 129.0, $128.6,128.4,128.2$, 127.7, 127.5, 126.5, 125.9, 125.7, 125.3, 111.0, 107.6, 55.6. HRMS (+ESI) Calcd for $\mathrm{C}_{30} \mathrm{H}_{21} \mathrm{O}_{3}[\mathrm{M}+\mathrm{H}]^{+}$: 429.1491; found: 429.1477. 

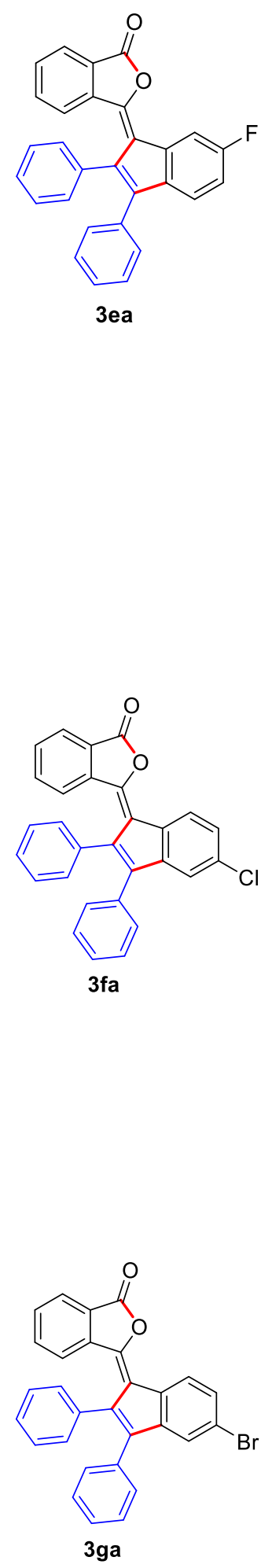

(E)-3-(6-Fluoro-2,3-diphenyl-1H-inden-1-ylidene)isobenzofuran-

1(3H)-one (3ea): The title compound was prepared by following the general procedure 2.2 and obtained as dark orange solid with $63 \mathrm{mg}$ (76\%) yield. M.p.: 226-228 ${ }^{\circ} \mathrm{C} .{ }^{1} \mathrm{H}$ NMR $\left(500 \mathrm{MHz}, \mathrm{CDCl}_{3}\right) \delta 8.29(\mathrm{dd}$, $J=10.0,2.4 \mathrm{~Hz}, 1 \mathrm{H}), 7.93(\mathrm{~d}, J=7.6 \mathrm{~Hz}, 1 \mathrm{H}), 7.43(\mathrm{t}, J=7.5 \mathrm{~Hz}, 1 \mathrm{H})$, 7.36-7.29 (m, 6H), 7.28-7.25 (m, 2H), 7.24-7.21 (m, 3H), $7.13(\mathrm{~d}, J=$ $8.4 \mathrm{~Hz}, 1 \mathrm{H}), 7.00(\mathrm{t}, J=8.6 \mathrm{~Hz}, 1 \mathrm{H}), 5.73(\mathrm{~d}, J=8.2 \mathrm{~Hz}, 1 \mathrm{H}) .{ }^{13} \mathrm{C} \mathrm{NMR}$ $\left(125 \mathrm{MHz}, \mathrm{CDCl}_{3}\right) \delta 165.8,162.3(\mathrm{~d}, J=242.5 \mathrm{~Hz}), 148.4,144.9,138.0$, $138.0,137.7,137.5,136.5,134.4,134.2,133.8,130.7,130.5,129.4$, 129.1, 128.2, 127.7 (d, $J=8.8 \mathrm{~Hz}$ ), 127.0, 126.0, 125.5, 121.3, 121.2, $115.0(\mathrm{~d}, J=23.8 \mathrm{~Hz}), 114.3(\mathrm{~d}, J=23.8 \mathrm{~Hz})$. HRMS (+ESI) Calcd for $\mathrm{C}_{29} \mathrm{H}_{18} \mathrm{FO}_{2}[\mathrm{M}+\mathrm{H}]^{+}:$417.1291; found: 417.1270.

\section{(E)-3-(5-Chloro-2,3-diphenyl-1H-inden-1-ylidene)isobenzofuran-}

1(3H)-one (3fa): The title compound was prepared by following the general procedure 2.2 and obtained as dark orange solid with $68 \mathrm{mg}$ (78\%) yield. M.p.: $281-284{ }^{\circ} \mathrm{C} .{ }^{1} \mathrm{H}$ NMR $\left(500 \mathrm{MHz}, \mathrm{CDCl}_{3}\right) \delta 8.46(\mathrm{~d}, J$ $=8.3 \mathrm{~Hz}, 1 \mathrm{H}), 7.93(\mathrm{~d}, J=7.6 \mathrm{~Hz}, 1 \mathrm{H}), 7.43(\mathrm{t}, J=7.4 \mathrm{~Hz}, 1 \mathrm{H}), 7.38$ $7.21(\mathrm{~m}, 12 \mathrm{H}), 7.13(\mathrm{t}, J=7.8 \mathrm{~Hz}, 1 \mathrm{H}), 5.71(\mathrm{~d}, J=8.2 \mathrm{~Hz}, 1 \mathrm{H}) .{ }^{13} \mathrm{C}$ NMR $\left(125 \mathrm{MHz}, \mathrm{CDCl}_{3}\right) \delta 166.0,148.2,144.4,143.6,137.5,136.3$, $136.2,134.2$, 133.8, 133.7, 130.6, 130.5, 129.4, 129.1, 128.3, 128.0, $127.9,127.7,126.9,126.3,125.9,125.5,125.0,120.9$. HRMS (+ESI) Calcd for $\mathrm{C}_{29} \mathrm{H}_{18} \mathrm{ClO}_{2}[\mathrm{M}+\mathrm{H}]^{+}$: 433.0995; found: 433.0984 .

\section{(E)-3-(5-Bromo-2,3-diphenyl-1H-inden-1-ylidene)isobenzofuran-}

1(3H)-one (3ga): The title compound was prepared by following the general procedure 2.2 and obtained as dark orange solid with $68 \mathrm{mg}$ (72\%) yield. M.p.: 293-295 ${ }^{\circ} \mathrm{C} .{ }^{1} \mathrm{H}$ NMR $\left(500 \mathrm{MHz}, \mathrm{CDCl}_{3}\right) \delta 8.40$ (d, J $=8.2 \mathrm{~Hz}, 1 \mathrm{H}), 7.93(\mathrm{~d}, J=7.5 \mathrm{~Hz}, 1 \mathrm{H}), 7.48-7.11(\mathrm{~m}, 14 \mathrm{H}), 5.71(\mathrm{~d}, J=$ $8.2 \mathrm{~Hz}, 1 \mathrm{H}) .{ }^{13} \mathrm{C} \mathrm{NMR}\left(125 \mathrm{MHz}, \mathrm{CDCl}_{3}\right) \delta 165.9,148.4,144.5,143.8$, $137.5,136.3$, 136.0, 134.6, 133.8, 133.7, 130.6, 130.6, 129.4, 129.3, $129.1,128.4$, 128.3, 127.9, 127.8, 126.9, 125.9, 125.5, 125.0, 123.8, 122.5. HRMS (+ESI) Calcd for $\mathrm{C}_{29} \mathrm{H}_{18} \mathrm{BrO}_{2}[\mathrm{M}+\mathrm{H}]^{+}$: 477.0490; found: 
477.0492.

\section{(E)-3-(2,3-Diphenyl-5-(trifluoromethoxy)-1H-inden-1-}

ylidene)isobenzofuran-1 $(3 H)$-one (3ha): The title compound was

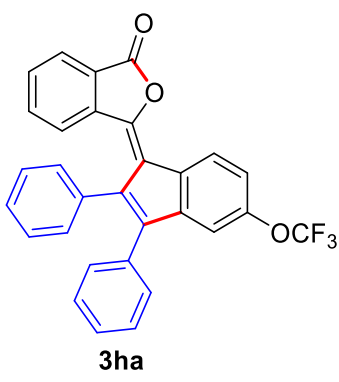
prepared by following the general procedure 2.2 and obtained as dark orange solid with $81 \mathrm{mg}$ (84\%) yield. M.p.: $204{ }^{\circ} \mathrm{C} .{ }^{1} \mathrm{H}$ NMR (500 MHz, $\left.\mathrm{CDCl}_{3}\right) \delta 8.56(\mathrm{~d}, J=8.3 \mathrm{~Hz}, 1 \mathrm{H}), 7.94(\mathrm{~d}, J=7.6 \mathrm{~Hz}, 1 \mathrm{H}), 7.44(\mathrm{t}, J=$ $7.4 \mathrm{~Hz}, 1 \mathrm{H}), 7.38-7.13(\mathrm{~m}, 13 \mathrm{H}), 5.71(\mathrm{~d}, J=8.2 \mathrm{~Hz}, 1 \mathrm{H}) .{ }^{13} \mathrm{C} \mathrm{NMR}$ $\left(126 \mathrm{MHz}, \mathrm{CDCl}_{3}\right) \delta 165.9,149.3,148.4,144.3,143.7,137.4,136.6$, $136.3,134.3,133.85,133.7,130.6,130.6,129.4,129.2$, 128.4, 128.1, $128.0,127.9$ (q, $J=287.5 \mathrm{~Hz}), 127.0,126.0,125.5,124.7,118.6,113.6$. HRMS (+ESI) Calcd for $\mathrm{C}_{30} \mathrm{H}_{18} \mathrm{~F}_{3} \mathrm{O}_{3}[\mathrm{M}+\mathrm{H}]^{+}$: 483.1208; found: 483.1207.

\section{(E)-3-(2,3-Di-p-Tolyl-1H-inden-1-ylidene)isobenzofuran-1(3H)-one}

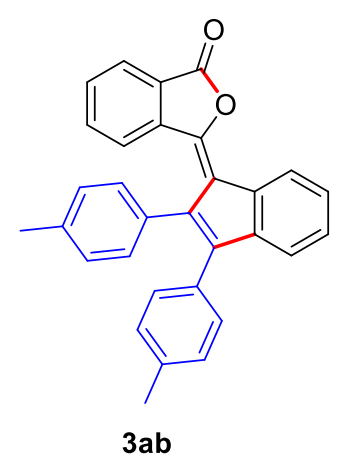

(3ab): The title compound was prepared by following the general procedure 2.2 and obtained as dark orange solid with $74 \mathrm{mg}(87 \%)$ yield. M.p.: $168{ }^{\circ} \mathrm{C} .{ }^{1} \mathrm{H}$ NMR $\left(500 \mathrm{MHz}, \mathrm{CDCl}_{3}\right) \delta 8.55(\mathrm{~d}, J=7.5 \mathrm{~Hz}, 1 \mathrm{H})$, $7.92(\mathrm{~d}, J=7.6 \mathrm{~Hz}, 1 \mathrm{H}), 7.41(\mathrm{t}, J=7.5 \mathrm{~Hz}, 1 \mathrm{H}), 7.35-7.29(\mathrm{~m}, 3 \mathrm{H})$, 7.17- $7.11(\mathrm{~m}, 9 \mathrm{H}), 5.77$ (d, $J=8.2 \mathrm{~Hz}, 1 \mathrm{H}), 2.42$ (s, 3H), 2.38 (s, 3H). ${ }^{13} \mathrm{C}$ NMR (125 MHz, $\left.\mathrm{CDCl}_{3}\right) \delta 166.3,147.5$ 145.3, 142.3, 137.8, 137.4, $137.2,136.1,134.5,133.8,133.5,131.4,130.6,130.1,129.7,129.4$, 128.9, 128.1, 127.2, 127.0, 126.6, 126.5, 125.8, 125.3, 120.7, 21.4, 21.3.

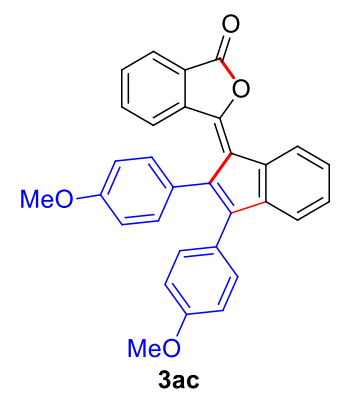

(E)-3-(2,3-bis(4-Methoxyphenyl)-1H-inden-1-ylidene)isobenzofuran1(3H)-one (3ac): The title compound was prepared by following the general procedure 2.2 and obtained as dark orange solid with $70 \mathrm{mg}$ (76\%) yield. M.p.: $219-223{ }^{\circ} \mathrm{C} .{ }^{1} \mathrm{H}$ NMR $\left(500 \mathrm{MHz}, \mathrm{CDCl}_{3}\right) \delta 8.54(\mathrm{~d}, J$ $=7.6 \mathrm{~Hz}, 1 \mathrm{H}), 7.92(\mathrm{~d}, J=7.5 \mathrm{~Hz}, 1 \mathrm{H}), 7.42(\mathrm{t}, J=7.5 \mathrm{~Hz}, 1 \mathrm{H}), 7.36-$ $7.30(\mathrm{~m}, 3 \mathrm{H}), 7.18(\mathrm{t}, J=8.5 \mathrm{~Hz}, 5 \mathrm{H}), 6.87(\mathrm{dd}, J=8.8,2.2 \mathrm{~Hz}, 4 \mathrm{H})$, $5.87(\mathrm{~d}, J=8.2 \mathrm{~Hz}, 1 \mathrm{H}), 3.86$ (s, 3H), 3.84 (s, 3H). ${ }^{13} \mathrm{C} \mathrm{NMR}(125 \mathrm{MHz}$, $\left.\mathrm{CDCl}_{3}\right) \delta 166.3,159.2,158.8,147.3,144.9,142.3,137.8,136.1,134.0$, $133.7,131.9,130.8,130.1,129.1,128.1,127.2,126.9,126.7,126.6$, 

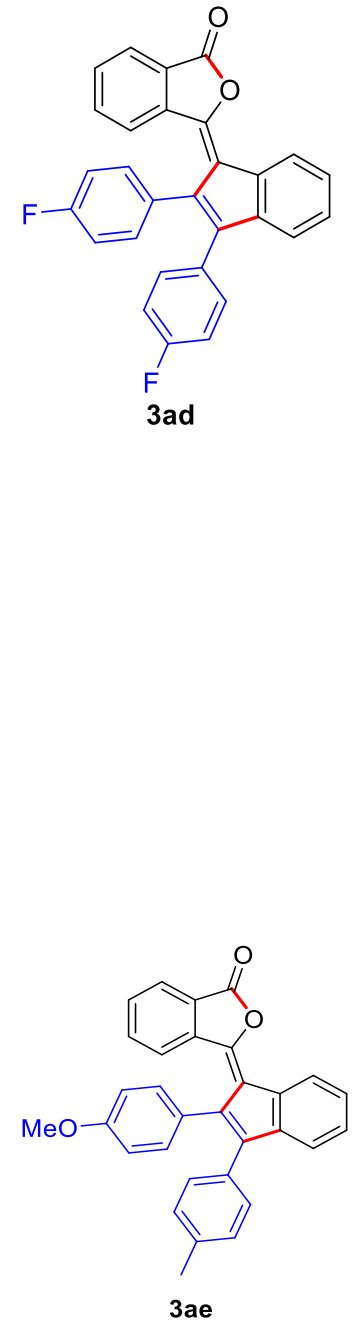

126.5, 125.8, 125.4, 120.6, 114.5, 113.6, 55.4, 55.2. HRMS (+ESI) Calcd for $\mathrm{C}_{31} \mathrm{H}_{23} \mathrm{O}_{4}[\mathrm{M}+\mathrm{H}]^{+}$: 459.1596; found: 459.1613 .

\section{(E)-3-(2,3-bis(4-Fluorophenyl)-1H-inden-1-ylidene)isobenzofuran-}

1(3H)-one (3ad): The title compound was prepared by following the general procedure 2.2 and obtained as dark orange solid with $70 \mathrm{mg}$ (81\%) yield. M.p.: 216-219 ${ }^{\circ} \mathrm{C} .{ }^{1} \mathrm{H}$ NMR $\left(500 \mathrm{MHz}, \mathrm{CDCl}_{3}\right) \delta 8.54$ (d, $J$ $=7.6 \mathrm{~Hz}, 1 \mathrm{H}), 7.94(\mathrm{~d}, J=7.7 \mathrm{~Hz}, 1 \mathrm{H}), 7.45(\mathrm{t}, J=7.5 \mathrm{~Hz}, 1 \mathrm{H}), 7.37(\mathrm{t}, J$ $=7.5 \mathrm{~Hz}, 1 \mathrm{H}), 7.33(\mathrm{~d}, J=7.4 \mathrm{~Hz}, 1 \mathrm{H}), 7.29-7.16(\mathrm{~m}, 6 \mathrm{H}), 7.04(\mathrm{t}, J=$ $8.6 \mathrm{~Hz}, 4 \mathrm{H}), 5.86(\mathrm{~d}, J=8.2 \mathrm{~Hz}, 1 \mathrm{H}) .{ }^{13} \mathrm{C} \mathrm{NMR}\left(125 \mathrm{MHz}, \mathrm{CDCl}_{3}\right) \delta$ $166.0,162.3(\mathrm{~d}, J=247.5 \mathrm{~Hz}), 162.0(\mathrm{~d}, J=247.5 \mathrm{~Hz}), 147.8,145.0$, $141.7,137.5,135.9,133.8,133.7,132.4(\mathrm{~d}, J=2.51 \mathrm{~Hz}), 132.1(\mathrm{~d}, J=$ $7.5 \mathrm{~Hz}), 131.0$ (d, $J=7.5 \mathrm{~Hz}), 130.4,130.0$ (d, $J=3.8 \mathrm{~Hz}), 128.3,127.3$, 127.1, 126.5, 126.0, 125.7, 125.6, 120.6, 116.0 (d, $J=20.0 \mathrm{~Hz}), 115.3$ (d, $J=21.3 \mathrm{~Hz}$ ). HRMS (+ESI) Calcd for $\mathrm{C}_{29} \mathrm{H}_{17} \mathrm{~F}_{2} \mathrm{O}_{2}[\mathrm{M}+\mathrm{H}]^{+}:$435.1197; found: 435.1196.

\section{Mixture of (E)-3-(3-(4-Methoxyphenyl)-2-(p-tolyl)-1H-inden-1-} ylidene)isobenzofuran-1(3H)-one and (E)-3-(2-(4-methoxyphenyl)-3(p-tolyl)-1H-inden-1-ylidene)isobenzofuran-1(3H)-one $\quad(1: 2) \quad(3 a e):$

The title compound was prepared by following the general procedure 2.2 and obtained as dark orange solid with $66 \mathrm{mg}$ (75\%) yield. M.p.: 208$209{ }^{\circ} \mathrm{C} .{ }^{1} \mathrm{H} \mathrm{NMR}\left(500 \mathrm{MHz}, \mathrm{CDCl}_{3}\right) \delta 8.55(\mathrm{dd}, J=7.5,3.3 \mathrm{~Hz}, 1 \mathrm{H})$, 7.9-7.89 (m, 1H), 7.41 (t, $J=7.2 \mathrm{~Hz}, 1 \mathrm{H}), 7.37-7.11(\mathrm{~m}, 10 \mathrm{H}), 6.87$ (d, $J$ $=8.6 \mathrm{~Hz}, 2 \mathrm{H}), 5.89(\mathrm{~d}, J=8.2 \mathrm{~Hz}, 0.3 \mathrm{H}), 5.78(\mathrm{~d}, J=8.1 \mathrm{~Hz}, 0.7 \mathrm{H}), 3.86$ $(\mathrm{s}, 0.9 \mathrm{H}), 3.84(\mathrm{~s}, 2.1 \mathrm{H}), 2.42(\mathrm{~s}, 2.1 \mathrm{H}), 2.38(\mathrm{~s}, 0.9 \mathrm{H}) .{ }^{13} \mathrm{C}$ NMR $(126$ $\left.\mathrm{MHz} \mathrm{CDCl}_{3}\right) \delta 166.3,158.9,147.3,145.0,142.3,137.8,137.4,137.2$, $136.1,134.3,133.8,133.7,133.5,131.8,130.8,130.6,130.1,129.7$, 129.4, 128. 9, 128.1, 127.2, 127.0, 126.7, 126.6, 126.5, 125.9, 125.8, 125.4, 125.3, 120.6, 114.5, 113.6, 55.4, 55.2, 21.4, 21.3. HRMS (+ESI) Calcd for $\mathrm{C}_{31} \mathrm{H}_{23} \mathrm{O}_{3}[\mathrm{M}+\mathrm{H}]^{+}$: 443.1647; found: 443.1642 . 


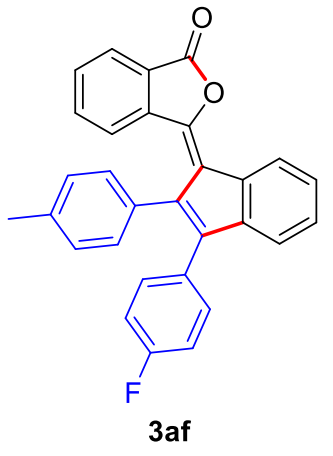

(E)-3-(2-(4-Fluorophenyl)-3-(p-tolyl)-1H-inden-1-

ylidene)isobenzofuran-1(3H)-one and $(E)$-3-(3-(4-fluorophenyl)-2-( $p$ tolyl)-1 $H$-inden-1-ylidene)isobenzofuran-1(3H)-one (1:1) (3af): The title compound was prepared by following the general procedure 2.2 and obtained as dark orange solid with $72 \mathrm{mg}(84 \%)$ yield. M.p.: $186{ }^{\circ} \mathrm{C} .{ }^{1} \mathrm{H}$ NMR $\left(500 \mathrm{MHz}, \mathrm{CDCl}_{3}\right) \delta 8.55(\mathrm{~d}, J=5.2 \mathrm{~Hz}, 1 \mathrm{H}), 7.97-7.89$ (t, $J=8.5$ $\mathrm{Hz}, 1 \mathrm{H}), 7.47-7.00(\mathrm{~m}, 13 \mathrm{H}), 5.86(\mathrm{~d}, J=8.2 \mathrm{~Hz}, 0.5 \mathrm{H}), 5.78$ (d, $J=8.2$ $\mathrm{Hz}, 0.5 \mathrm{H}), 2.42$ (s, 1.5H), 2.39 (s, 1.5H). $\left.{ }^{13} \mathrm{C} \mathrm{NMR} \mathrm{(125} \mathrm{MHz,} \mathrm{CDCl}_{3}\right) \delta$ 166.2, 166.1, $162.2(\mathrm{~d}, J=246.3 \mathrm{~Hz}), 161.9$ (d, $J=245.0 \mathrm{~Hz}), 147.8$, $147.4,146.2,144.2,142.0,137.7,137.6,137.5,136.0,135.1,133.7$, 133.6, 133.5, 133.2, 132.9, $132.2(\mathrm{~d}, J=7.5 \mathrm{~Hz}), 131.1(\mathrm{~d}, J=7.5 \mathrm{~Hz})$, $130.5,130.4,130.3,129.8,129.3,129.0,128.0(\mathrm{~d}, J=5.0 \mathrm{~Hz}), 127.3$, 127.2, 127.0, 126.9, 126.8, 126.5, 126.1, 125.8 (d, $J=6.3 \mathrm{~Hz}), 125.6$, 125.4, 120.9, 120.4, 116.0 (d, $J=21.3 \mathrm{~Hz}), 115.1$ (d, $J=21.3 \mathrm{~Hz}), 21.4$, 21.3. HRMS (+ESI) Calcd for $\mathrm{C}_{30} \mathrm{H}_{20} \mathrm{FO}_{2}[\mathrm{M}+\mathrm{H}]^{+}$: 431.1447; found: 431.1446 . 

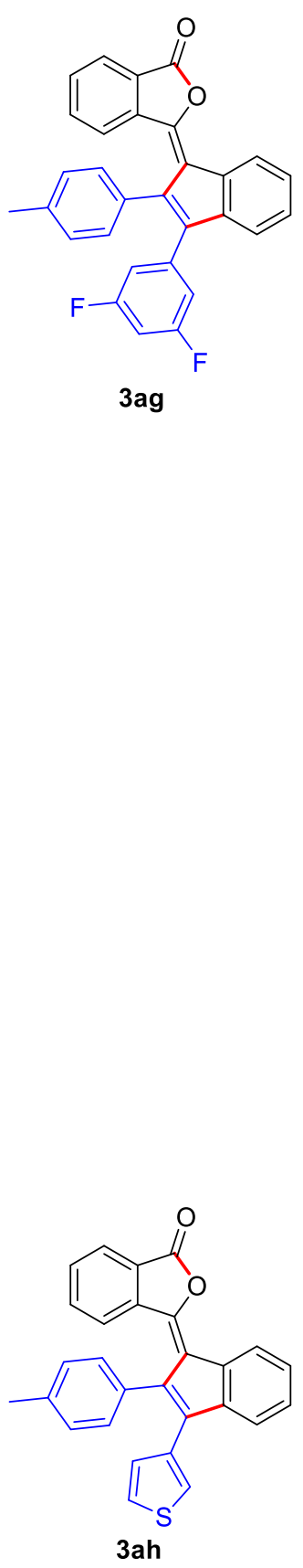

(E)-3-(2-(3,5-Difluorophenyl)-3-(p-tolyl)-1H-inden-1-

ylidene)isobenzofuran-1(3H)-one and (E)-3-(3-(3,5-difluorophenyl)2-(p-tolyl)-1H-inden-1-ylidene)isobenzofuran-1(3H)-one (1:1) (3ag):

The title compound was prepared by following the general procedure 2.2 and obtained as dark orange solid with $73 \mathrm{mg}(82 \%)$ yield. M.p.: $230{ }^{\circ} \mathrm{C}$. ${ }^{1} \mathrm{H}$ NMR $\left(500 \mathrm{MHz}, \mathrm{CDCl}_{3}\right) \delta 8.55(\mathrm{t}, J=7.5 \mathrm{~Hz}, 1 \mathrm{H}), 7.97(\mathrm{~d}, J=7.7$ $\mathrm{Hz}, 1 \mathrm{H}), 7.49(\mathrm{t}, J=7.5 \mathrm{~Hz}, 0.5 \mathrm{H}), 7.44(\mathrm{t}, J=7.5 \mathrm{~Hz}, 0.5 \mathrm{H}), 7.40-7.09$ $(\mathrm{m}, 8 \mathrm{H}), 6.85-6.79(\mathrm{~m}, 1.5 \mathrm{H}), 6.78-6.70(\mathrm{~m}, 1.5 \mathrm{H}), 6.01(\mathrm{~d}, J=8.2 \mathrm{~Hz}$, $0.5 \mathrm{H}), 5.78(\mathrm{~d}, J=8.2 \mathrm{~Hz}, 0.5 \mathrm{H}), 2.43(\mathrm{~s}, 1.5 \mathrm{H}), 2.40(\mathrm{~s}, 1.5 \mathrm{H}) .{ }^{13} \mathrm{C} \mathrm{NMR}$ $\left(125 \mathrm{MHz}, \mathrm{CDCl}_{3}\right) \delta 166.0,165.9,163.2(\mathrm{~d}, J=248.8 \mathrm{~Hz}), 163.0(\mathrm{~d}, J=$ $248.8 \mathrm{~Hz}), 162.7(\mathrm{~d}, J=247.5 \mathrm{~Hz}), 162.6(\mathrm{~d}, J=247.5 \mathrm{~Hz}), 148.6,147.5$, $147.2,141.6,141.2,140.2,138.1,137.9,137.6,137.5,136.0,135.7$, $133.8,133.7,132.9,130.7,130.6,130.5,130.3,130.0,129.2,129.1$, $128.4,128.3,127.4,127.3,127.2,127.1,127.0,126.0,125.8,125.7$, 125.5, 125.2, 121.2, 120.2, $113.8(\mathrm{~d}, J=18.8 \mathrm{~Hz}), 113.7(\mathrm{~d}, J=18.8 \mathrm{~Hz})$, $112.4(\mathrm{~d}, J=20.0 \mathrm{~Hz}), 112.3(\mathrm{~d}, J=20.0 \mathrm{~Hz}), 102.9$ (t, $J=26.3 \mathrm{~Hz})$, $102.8(\mathrm{t}, J=26.3 \mathrm{~Hz}), 21.4,21.3$. HRMS (+ESI) Calcd for $\mathrm{C}_{30} \mathrm{H}_{19} \mathrm{~F}_{2} \mathrm{O}_{2}$ $[\mathrm{M}+\mathrm{H}]^{+}$: 449.1353; found: 449.1356 .

Mixture of $\quad(E)-3-(3-(T h i o p h e n-3-y l)-2-(p-t o l y l)-1 H$-inden-1ylidene)isobenzofuran-1(3H)-one and (E)-3-(2-(thiophen-3-yl)-3-(ptolyl)-1H-inden-1-ylidene)isobenzofuran-1(3H)-one $\quad(\sim 1.5: 1) \quad(3 \mathrm{ah})$ :

The title compound was prepared by following the general procedure 2.2 and obtained as dark orange semi solid with $59 \mathrm{mg}(71 \%)$ yield. ${ }^{1} \mathrm{H}$ NMR $\left(500 \mathrm{MHz} \mathrm{CDCl}_{3}\right) \delta 8.56(\mathrm{~d}, J=7.5 \mathrm{~Hz}, 0.6 \mathrm{H}), 8.52(\mathrm{~d}, J=7.4 \mathrm{~Hz}, 0.4$ H),8.40-8.20(m, 1.5H), 8.0-7.06(m, 13H), $6.92(\mathrm{~d}, J=5.0 \mathrm{~Hz}, 0.4 \mathrm{H})$, $6.87(\mathrm{~d}, J=5.0 \mathrm{~Hz}, 0.6 \mathrm{H}), 5.98(\mathrm{~d}, J=8.9 \mathrm{~Hz}, 0.4 \mathrm{H}), 5.77(\mathrm{~d}, J=8.2 \mathrm{~Hz}$, $0.6 \mathrm{H}), 2.45$ (s, 1.8H), 2.39 (s, 1.24H). $\left.{ }^{13} \mathrm{C} \mathrm{NMR} \mathrm{(125} \mathrm{MHz,} \mathrm{CDCl}_{3}\right) \delta$ $181.5,175.6,166.3,156.5,147.5,141.7,140.3,137.9,137.8,137.5$, $136.1,136.0,134.8,134.7,134.3,134.0,133.6,133.3,132.4,131.3$, $130.5,130.3,130.2,130.0,129.9,129.7,129.1,128.9,128.6,128.2$, $127.3,127.1,127.0,126.9,126.8,126.7,126.6,126.4,126.3,126.2$, 


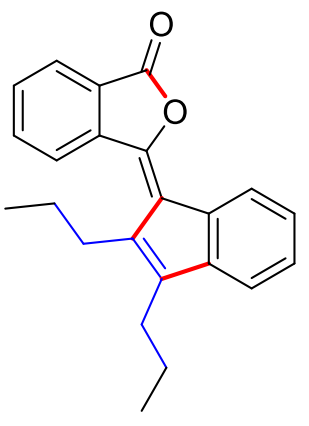

3ai

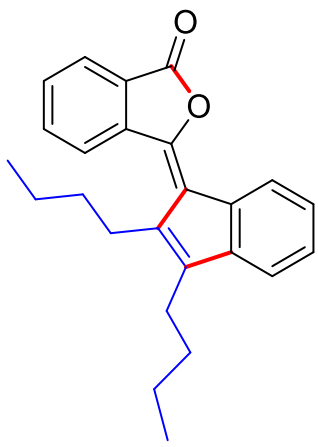

3aj

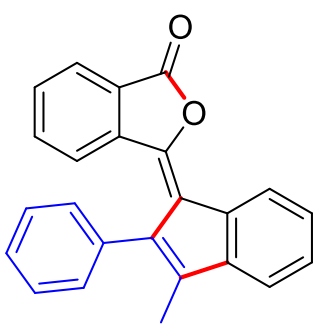

3ak
$125.8,125.4,124.9,124.8,124.1,120.8,120.6,113.0,21.4$. HRMS (+ESI) Calcd for $\mathrm{C}_{28} \mathrm{H}_{19} \mathrm{O}_{2} \mathrm{~S}[\mathrm{M}+\mathrm{H}]^{+}$: 419.1106 ; found: 419.1102 .

\section{(Z)-3-(2,3-Dipropyl-1H-inden-1-ylidene)isobenzofuran-1(3H)-one}

(3ai): The title compound was prepared by following the general procedure 2.2 and obtained as dark orange solid with $52 \mathrm{mg}$ (79\%) yield. M.p.: $79-83{ }^{\circ} \mathrm{C} .{ }^{1} \mathrm{H}$ NMR $\left(500 \mathrm{MHz}, \mathrm{CDCl}_{3}\right) \delta 8.60(\mathrm{~d}, J=8.0 \mathrm{~Hz}, 1 \mathrm{H})$, $8.10(\mathrm{~d}, J=7.6 \mathrm{~Hz}, 1 \mathrm{H}), 8.05(\mathrm{~d}, J=7.6 \mathrm{~Hz}, 1 \mathrm{H}), 7.78(\mathrm{t}, J=8.2 \mathrm{~Hz}$, $1 \mathrm{H}), 7.64(\mathrm{t}, J=7.3 \mathrm{~Hz}, 1 \mathrm{H}), 7.28-7.25(\mathrm{~m}, 2 \mathrm{H}), 7.17(\mathrm{t}, J=7.2 \mathrm{~Hz}, 1 \mathrm{H})$, $2.81(\mathrm{t}, J=7.7 \mathrm{~Hz}, 2 \mathrm{H}), 2.60$ (t, $J=7.8 \mathrm{~Hz}, 2 \mathrm{H}), 1.68-1.63(\mathrm{~m}, 4 \mathrm{H}), 1.07$ $(\mathrm{m}, 6 \mathrm{H}) .{ }^{13} \mathrm{C}$ NMR $\left(125 \mathrm{MHz}, \mathrm{CDCl}_{3}\right) \delta 166.0,145.0,144.5,143.1$, 139.0, 138.7, 134.7, 134.1, 130.6, 128.2, 126.9, 126.1, 125.9, 124.9, 124.5, 123.0, 119.2, 29.5, 27.6, 24.2, 22.4, 14.5, 14.4. HRMS (+ESI) Calcd for $\mathrm{C}_{23} \mathrm{H}_{23} \mathrm{O}_{2}[\mathrm{M}+\mathrm{H}]^{+}:$331.1698; found: 331.1694 .

\section{(Z)-3-(2,3-Dibutyl-1 $H$-inden-1-ylidene)isobenzofuran-1(3H)-one}

(3aj): The title compound was prepared by following the general procedure 2.2 and obtained as semi-solid with $57 \mathrm{mg}(80 \%)$ yield. ${ }^{1} \mathrm{H}$ NMR $\left(500 \mathrm{MHz}, \mathrm{CDCl}_{3}\right) \delta 8.60(\mathrm{~d}, J=8.1 \mathrm{~Hz}, 1 \mathrm{H}), 8.10(\mathrm{dd}, J=7.6$, $0.9 \mathrm{~Hz}, 1 \mathrm{H}), 8.05(\mathrm{~d}, J=7.7 \mathrm{~Hz}, 1 \mathrm{H}), 7.79(\mathrm{t}, J=8.2 \mathrm{~Hz}, 1 \mathrm{H}), 7.64(\mathrm{t}, J=$ $7.5 \mathrm{~Hz}, 1 \mathrm{H}), 7.28-7.24(\mathrm{~m}, 2 \mathrm{H}), 7.14(\mathrm{t}, J=7.2 \mathrm{~Hz}, 1 \mathrm{H}), 2.83(\mathrm{t}, J=7.6$ $\mathrm{Hz}, 2 \mathrm{H}), 2.60$ (t, $J=7.8 \mathrm{~Hz}, 2 \mathrm{H}), 1.70-1.42(\mathrm{~m}, 8 \mathrm{H}), 0.99$ (t, $J=7.3 \mathrm{~Hz}$, $6 \mathrm{H}) .{ }^{13} \mathrm{C}$ NMR $\left(125 \mathrm{MHz}, \mathrm{CDCl}_{3}\right) \delta 166.0,145.0,143.2,139.1,138.7$, 134.7, 134.1, 130.6, 128.2, 126.9, 126.1, 125.9, 124.9, 124.4, 123.0, 119.2, 33.1, 31.3, 27.3, 25.3, 23.2, 23.0, 14.1. HRMS (+ESI) Calcd for $\mathrm{C}_{25} \mathrm{H}_{27} \mathrm{O}_{2}[\mathrm{M}+\mathrm{H}]^{+}:$359.2011; found: 413.2014 .

(E)-3-(3-Methyl-2-phenyl-1 $H$-inden-1-ylidene)isobenzofuran-1(3H)-

one (3ak): The title compound was prepared by following the general procedure 2.2 and obtained as dark orange solid with $47 \mathrm{mg}$ (70\%) yield. M.p.: $123-125{ }^{\circ} \mathrm{C} .{ }^{1} \mathrm{H}$ NMR $\left(500 \mathrm{MHz}, \mathrm{CDCl}_{3}\right) \delta 8.48(\mathrm{~d}, J=7.0 \mathrm{~Hz}$, $1 \mathrm{H}), 7.89(\mathrm{~d}, J=7.6 \mathrm{~Hz}, 1 \mathrm{H}), 7.50-7.32(\mathrm{~m}, 9 \mathrm{H}), 7.11(\mathrm{t}, J=7.3 \mathrm{~Hz}$, $1 \mathrm{H}), 5.75(\mathrm{~d}, J=8.2 \mathrm{~Hz}, 1 \mathrm{H}), 2.17$ (s, 3H). ${ }^{13} \mathrm{C} \mathrm{NMR}\left(125 \mathrm{MHz}, \mathrm{CDCl}_{3}\right)$ $\delta 166.4,146.0,142.9,142.7,137.8,137.3,136.0,134.2,133.8,133.6$, 
$130.5,130.3,130.0,129.4,129.2,128.3,127.7,127.0,126.7,126.1$, 125.8, 125.3, 119.3, 11.7. HRMS (+ESI) Calcd for $\mathrm{C}_{24} \mathrm{H}_{17} \mathrm{O}_{2}[\mathrm{M}+\mathrm{H}]^{+}$: 337.1229; found: 337.1240 .
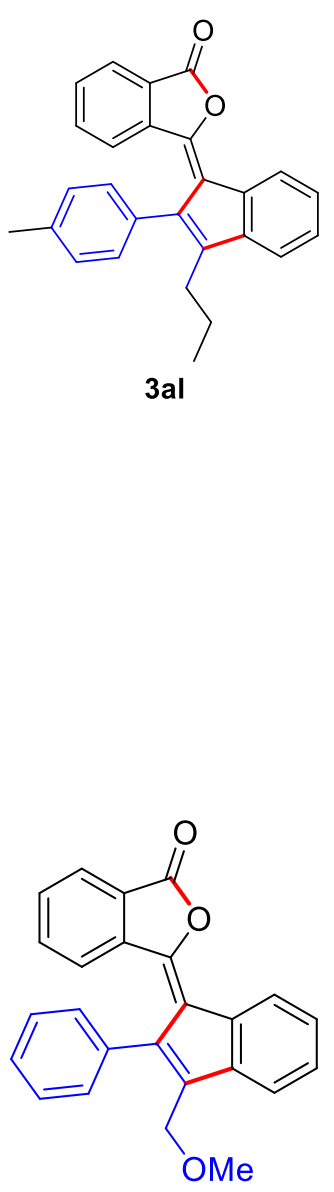

3am

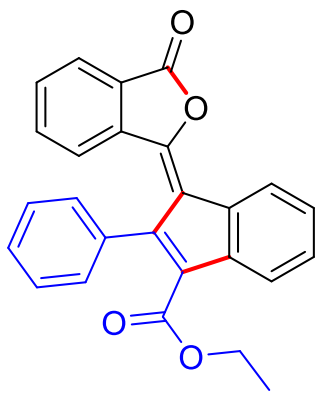

3an
(E)-3-(3-Propyl-2-(p-tolyl)-1H-inden-1-ylidene)isobenzofuran-1(3H)one (3al): The title compound was prepared by following the general procedure 2.2 and obtained as dark orange solid with $60 \mathrm{mg}(80 \%)$ yield. M.p.: $167-169{ }^{\circ} \mathrm{C} .{ }^{1} \mathrm{H}$ NMR $\left(500 \mathrm{MHz}, \mathrm{CDCl}_{3}\right) \delta 8.49(\mathrm{~d}, J=7.2 \mathrm{~Hz}$, 1H), $7.90(\mathrm{~d}, J=7.6 \mathrm{~Hz}, 1 \mathrm{H}), 7.40$ (t, $J=7.4 \mathrm{~Hz}, 1 \mathrm{H}), 7.38-7.24(\mathrm{~m}$, $7 \mathrm{H}), 7.13(\mathrm{t}, J=7.7 \mathrm{~Hz}, 1 \mathrm{H}), 5.76(\mathrm{~d}, J=8.2 \mathrm{~Hz}, 1 \mathrm{H}), 2.52$ (t, $J=7.8 \mathrm{~Hz}$, 2H), 2.49 (s, 3H), 1.69-1.64 (m, 2H), $0.95(\mathrm{t}, J=7.4 \mathrm{~Hz}, 3 \mathrm{H}) .{ }^{13} \mathrm{C} \mathrm{NMR}$ $\left(125 \mathrm{MHz}, \mathrm{CDCl}_{3}\right) \delta 166.4,147.1,146.2,142.1,137.9,137.5,136.2$, $134.3,134.3,133.5,130.1,130.0,129.8,128.2,127.2$, 126.4, 126.4, 125.8, 125.2, 119.5, 28.4, 22.7, 21.3, 14.5. HRMS (+ESI) Calcd for $\mathrm{C}_{27} \mathrm{H}_{23} \mathrm{O}_{2}[\mathrm{M}+\mathrm{H}]^{+}:$379.1698; found: 379.1711 .

\section{(E)-3-(3-(Methoxymethyl)-2-phenyl-1H-inden-1-}

ylidene)isobenzofuran-1(3H)-one (3am): The title compound was prepared by following the general procedure 2.2 and obtained as dark orange solid with $53 \mathrm{mg}$ (73\%) yield. M.p.: $66{ }^{\circ} \mathrm{C}$. ${ }^{1} \mathrm{H}$ NMR $(500 \mathrm{MHz}$, $\left.\mathrm{CDCl}_{3}\right) \delta 8.49(\mathrm{~d}, J=7.0 \mathrm{~Hz}, 1 \mathrm{H}), 7.91(\mathrm{~d}, J=7.6 \mathrm{~Hz}, 1 \mathrm{H}), 7.58-7.30$ (m, 9H), $7.13(\mathrm{t}, J=8.4 \mathrm{~Hz}, 1 \mathrm{H}), 5.72(\mathrm{~d}, J=8.2 \mathrm{~Hz}, 1 \mathrm{H}), 4.41(\mathrm{~s}, 2 \mathrm{H})$, $3.36(\mathrm{~s}, 3 \mathrm{H}) .{ }^{13} \mathrm{C}$ NMR $\left(125 \mathrm{MHz}, \mathrm{CDCl}_{3}\right) \delta 166.1,147.9,141.7,141.1$, $137.6,136.4,135.7,133.8,130.4,130.2$, 129.2, 128.5, 128.2, 127.2, $126.8,126.4,125.9,125.4,120.6,66.3,58.2$.

Ethyl (E)-1-(3-oxoisobenzofuran-1(3H)-ylidene)-2-phenyl-1H-indene3-carboxylate (3an): The title compound was prepared by following the general procedure 2.2 and obtained as dark orange solid with $54 \mathrm{mg}$ (69\%) yield. M.p.: $131-134{ }^{\circ} \mathrm{C} .{ }^{1} \mathrm{H}$ NMR $\left(500 \mathrm{MHz}, \mathrm{CDCl}_{3}\right) \delta 8.52(\mathrm{~d}, J$ $=7.2 \mathrm{~Hz}, 1 \mathrm{H}), 7.92(\mathrm{t}, J=9.7 \mathrm{~Hz}, 2 \mathrm{H}), 7.52-7.39(\mathrm{~m}, 8 \mathrm{H}), 7.15(\mathrm{t}, J=$ $7.6 \mathrm{~Hz}, 1 \mathrm{H}), 5.58(\mathrm{~d}, J=8.3 \mathrm{~Hz}, 1 \mathrm{H}), 4.20(\mathrm{q}, J=7.1 \mathrm{~Hz}, 2 \mathrm{H}), 1.13(\mathrm{t}, J$ $=6.8 \mathrm{~Hz}, 3 \mathrm{H}) .{ }^{13} \mathrm{C} \mathrm{NMR}\left(125 \mathrm{MHz}, \mathrm{CDCl}_{3}\right) \delta 165.5,164.6,151.2,144.0$, $138.9,137.3,136.7,135.1,134.9,134.1,131.1,129.8,128.9,128.6$, 
128.5, 127.4, 127.0, 126.1, 125.7, 125.6, 122.4, 60.5, 13.9. HRMS (+ESI) Calcd for $\mathrm{C}_{26} \mathrm{H}_{19} \mathrm{O}_{4}[\mathrm{M}+\mathrm{H}]^{+}:$395.1286; found: 395.1286.

\section{(E)-3-(2-Phenyl-3-(phenylethynyl)-1H-inden-1-}

ylidene)isobenzofuran-1(3H)-one (3ao): The title compound was

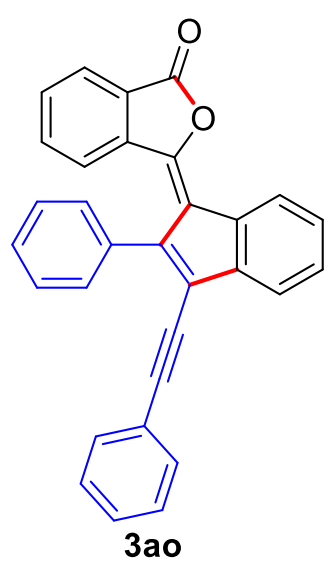
prepared by following the general procedure 2.2 and obtained as dark orange solid with $56 \mathrm{mg}$ (66\%) yield. M.p.: $198{ }^{\circ} \mathrm{C} .{ }^{1} \mathrm{H}$ NMR (500 MHz, $\left.\mathrm{CDCl}_{3}\right) \delta 8.47(\mathrm{~d}, J=7.5 \mathrm{~Hz}, 1 \mathrm{H}), 7.93(\mathrm{~d}, J=7.6 \mathrm{~Hz}, 1 \mathrm{H}), 7.63$ (d, $J=$ $7.2 \mathrm{~Hz}, 3 \mathrm{H}), 7.48(\mathrm{~d}, J=1.7 \mathrm{~Hz}, 4 \mathrm{H}), 7.44(\mathrm{~d}, J=7.2 \mathrm{~Hz}, 2 \mathrm{H}), 7.41(\mathrm{dd}$, $J=8.8,1.1 \mathrm{~Hz}, 2 \mathrm{H}), 7.37-7.36(\mathrm{~m}, 3 \mathrm{H}), 7.17(\mathrm{t}, J=7.7 \mathrm{~Hz}, 1 \mathrm{H}), 6.05(\mathrm{~d}$, $J=8.2 \mathrm{~Hz}, 1 \mathrm{H}) .{ }^{13} \mathrm{C}$ NMR $\left(125 \mathrm{MHz}, \mathrm{CDCl}_{3}\right) \delta 166.0,147.9,141.6$, $140.6,137.5,136.4,135.6,133.7,131.8,131.8,130.8,130.6,130.0$, $129.9,129.5,128.9,128.7,128.5,128.4,128.4,128.2,127.7,127.3$, 126.7, 125.8, 125.5, 123.1, 120.6, 100.1, 84.8. HRMS (+ESI) Calcd for $\mathrm{C}_{31} \mathrm{H}_{19} \mathrm{O}_{2}[\mathrm{M}+\mathrm{H}]^{+}: 423.1385$; found: 423.1383 .

\section{(E)-3-(2-(p-Tolyl)-3-(p-tolylethynyl)-1 $H$-inden-1-}

ylidene)isobenzofuran-1(3H)-one (3ap): The title compound was

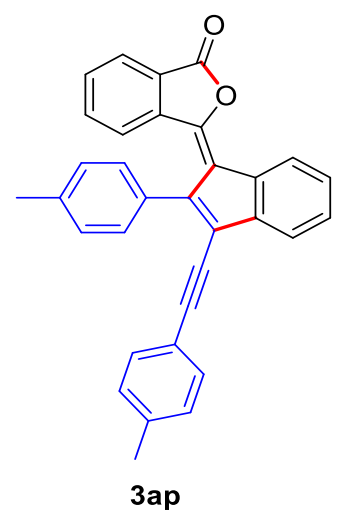

prepared by following the general procedure 2.2 and obtained as dark orange solid with $60 \mathrm{mg}$ (67\%) yield. M.p.: 220-224 ${ }^{\circ} \mathrm{C} .{ }^{1} \mathrm{H}$ NMR (500 $\left.\mathrm{MHz}, \mathrm{CDCl}_{3}\right) \delta 8.44(\mathrm{dd}, J=7.0,1.2 \mathrm{~Hz}, 1 \mathrm{H}), 7.93(\mathrm{~d}, J=7.7 \mathrm{~Hz}, 1 \mathrm{H})$, $7.60(\mathrm{dd}, J=7.1,0.9 \mathrm{~Hz}, 1 \mathrm{H}), 7.51(\mathrm{~d}, J=8.1 \mathrm{~Hz}, 2 \mathrm{H}), 7.46-7.16(\mathrm{~m}$, $10 \mathrm{H}), 6.12(\mathrm{~d}, J=8.2 \mathrm{~Hz}, 1 \mathrm{H}), 2.48$ (s, 3H), 2.40 (s, 3H). ${ }^{13} \mathrm{C}$ NMR $(125$ $\left.\mathrm{MHz}, \mathrm{CDCl}_{3}\right) \delta 166.1,147.7,141.2,140.8,139.0,138.1,137.6,135.6$, $133.6,133.4,131.7,130.5,129.8,129.6,129.2,128.5,127.5,127.1$, 126.9, 126.6, 125.7, 125.4, 125.2, 120.5, 120.1, 100.5, 84.4, 21.6, 21.5. HRMS (+ESI) Calcd for $\mathrm{C}_{33} \mathrm{H}_{23} \mathrm{O}_{2}[\mathrm{M}+\mathrm{H}]^{+}:$451.1698; found: 423.1696. 
(E)-3-(2-(4-Fluorophenyl)-3-((4-fluorophenyl)ethynyl)-1H-inden-1-

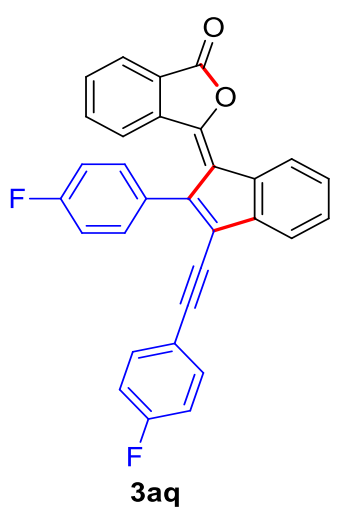

ylidene)isobenzofuran-1(3H)-one (3aq): The title compound was prepared by following the general procedure 2.2 using alkyne $2 \mathrm{~s}$ and obtained as orange liquid with $35 \mathrm{mg}(38 \%)$ yield. ${ }^{1} \mathrm{H}$ NMR $(500 \mathrm{MHz}$, $\left.\mathrm{CDCl}_{3}\right) \delta 8.44(\mathrm{~d}, J=6.9 \mathrm{~Hz}, 1 \mathrm{H}), 7.94(\mathrm{~d}, J=7.6 \mathrm{~Hz}, 1 \mathrm{H}), 7.64-7.53$ (m, 3H), 7.53-7.32 (m, 6H), $7.18(\mathrm{t}, J=8.7 \mathrm{~Hz}, 2 \mathrm{H}), 7.06(\mathrm{t}, J=8.7 \mathrm{~Hz}$, $2 \mathrm{H}), 6.13(\mathrm{~d}, J=8.2 \mathrm{~Hz}, 1 \mathrm{H}) .{ }^{13} \mathrm{C}$ NMR $\left(125 \mathrm{MHz}, \mathrm{CDCl}_{3}\right) \delta 165.8$, $162.7(\mathrm{~d}, J=233.8 \mathrm{~Hz}), 162.6(\mathrm{~d}, J=233.8 \mathrm{~Hz}), 147.8,140.2,137.2$, 135.4, 133.7, $133.6(\mathrm{~d}, J=8.8 \mathrm{~Hz}), 131.6(\mathrm{~d}, J=7.5 \mathrm{~Hz}), 130.7,128.5$, 127.7, 127.4, 127.3, 126.6, 126.3, 125.9, 125.7, 125.7, 124.7, 120.5, 115.9 (d, $J=28.8 \mathrm{~Hz}), 115.7$ (d, $J=21.2 \mathrm{~Hz}), 99.1,84.2$. HRMS (+ESI) Calcd for $\mathrm{C}_{31} \mathrm{H}_{17} \mathrm{~F}_{2} \mathrm{O}_{2}[\mathrm{M}+\mathrm{H}]^{+}$: 459.1197; found: 459.1186 .

\section{Reference:}

1. Verma, A.; Kumar, S. Org. Lett. 2016, 18, 4388-4391. 


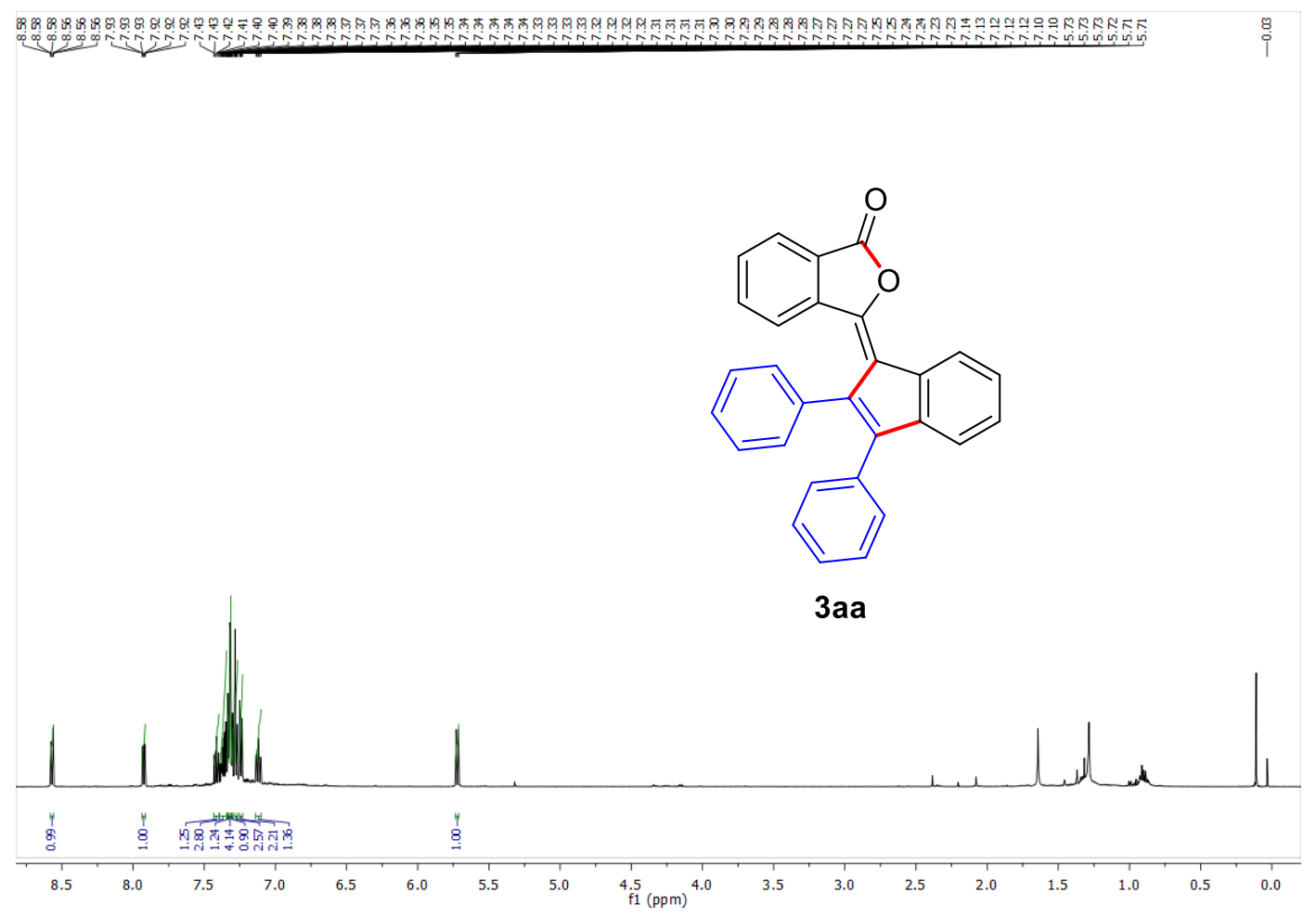

Expanded form of 3aa:

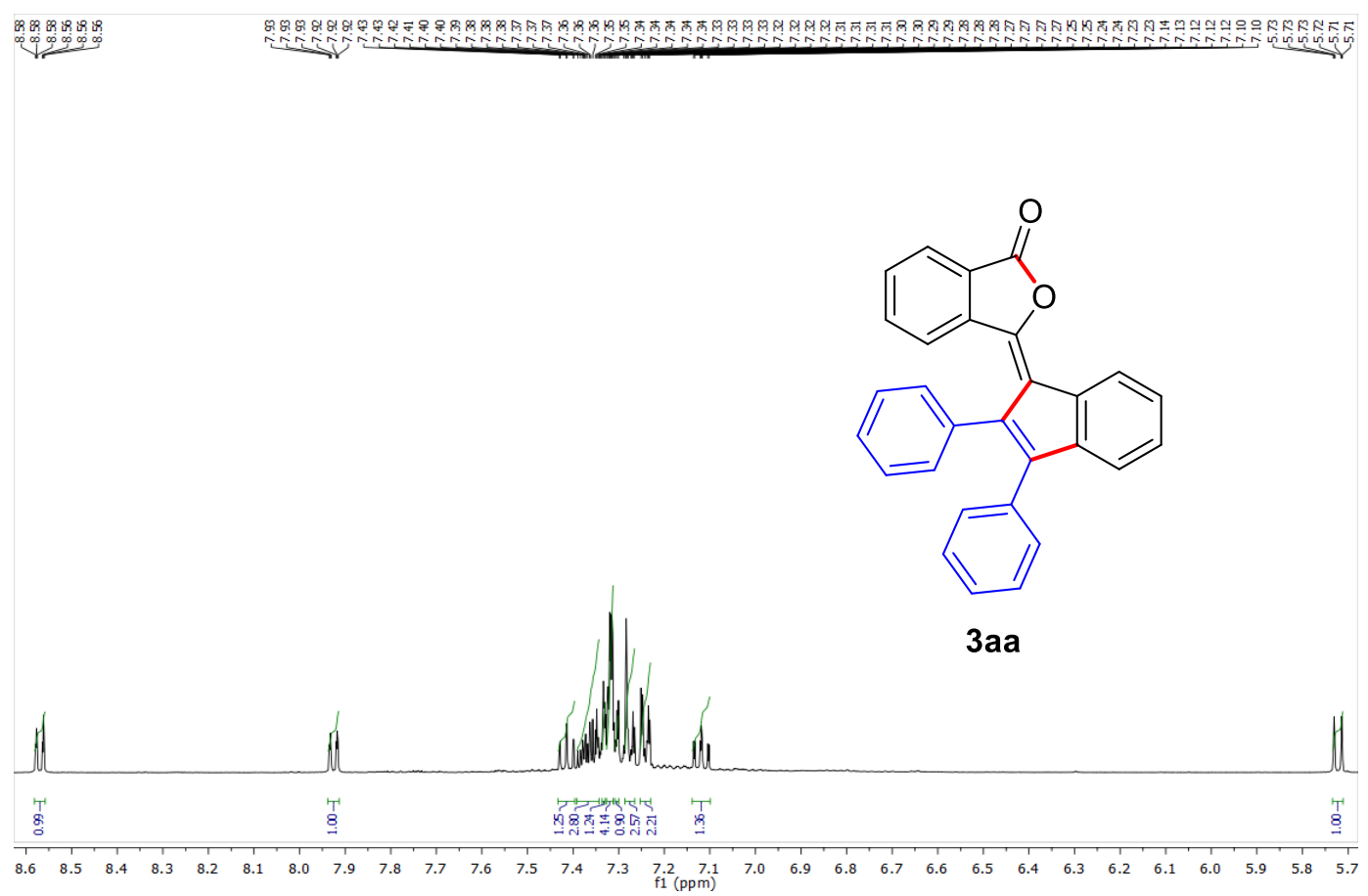



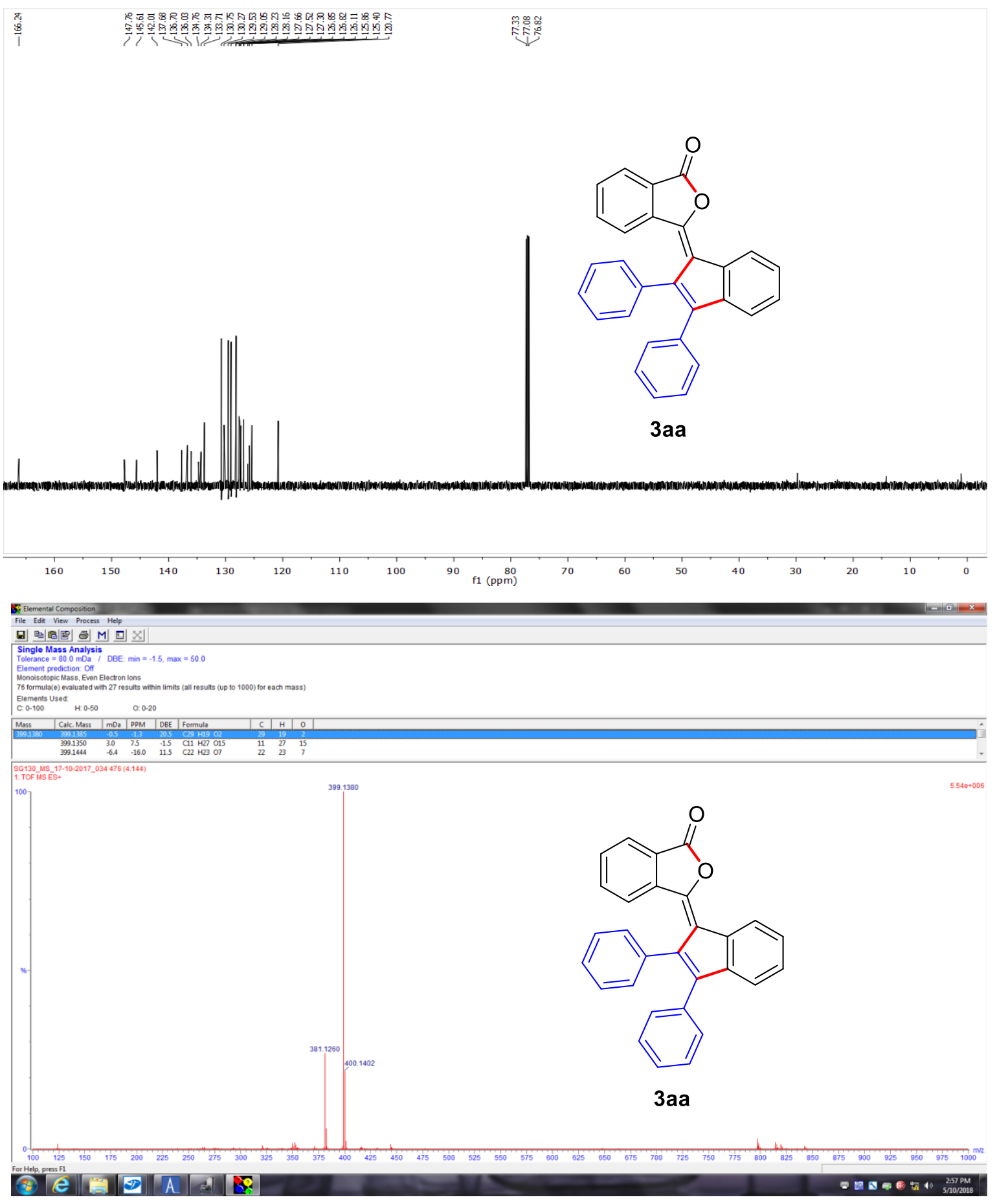


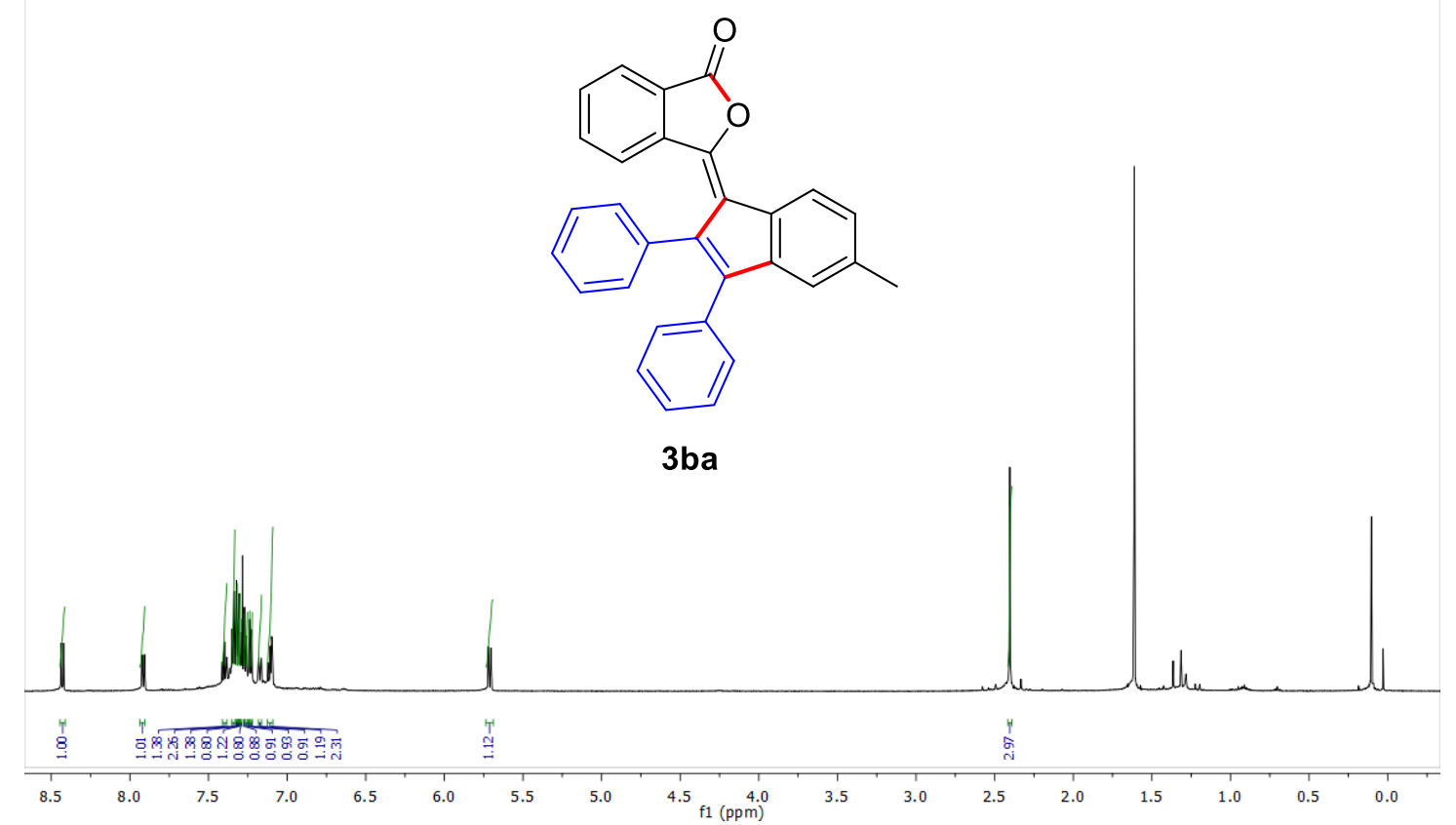

Expanded form of $3 \mathrm{ba}$ :

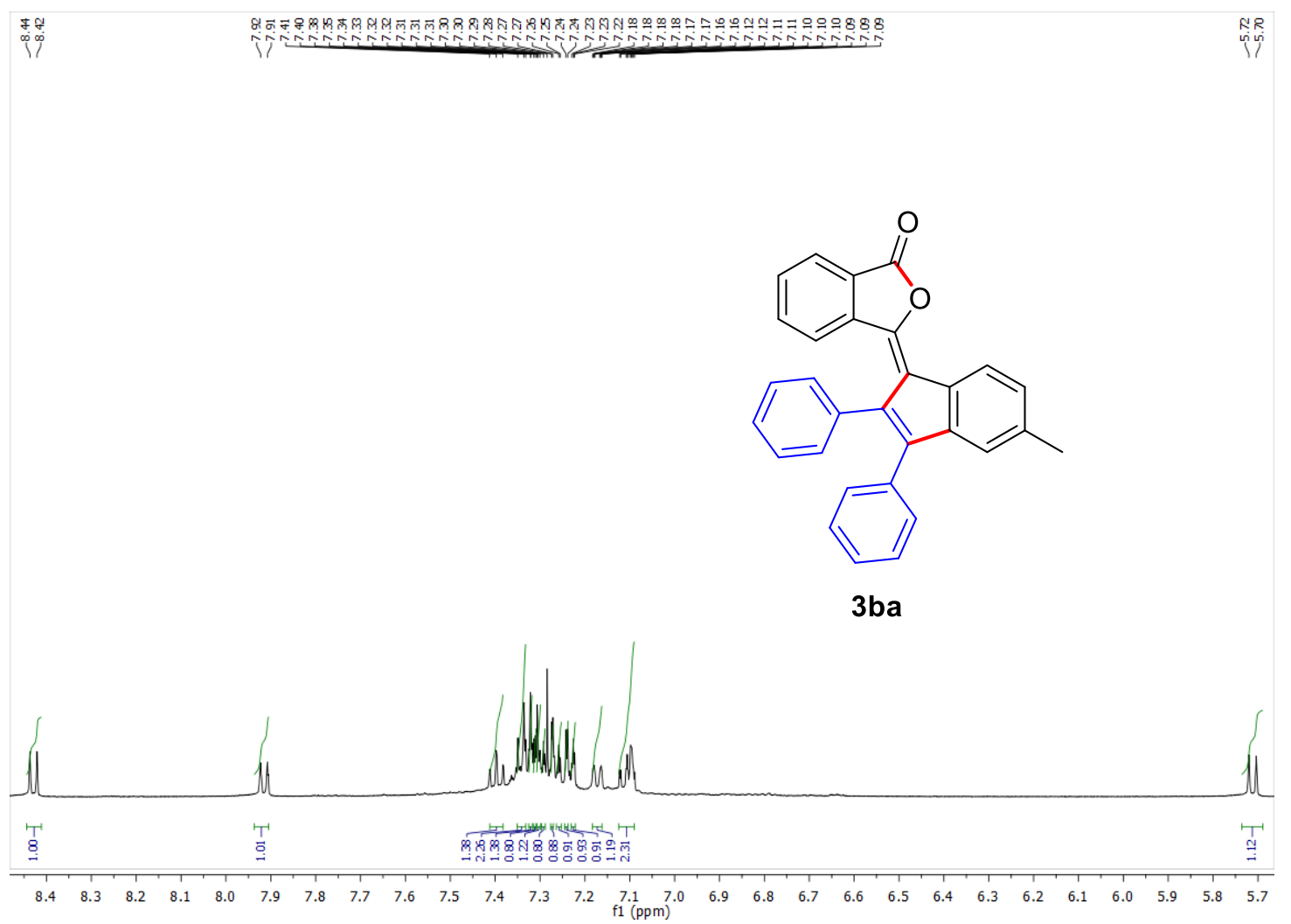



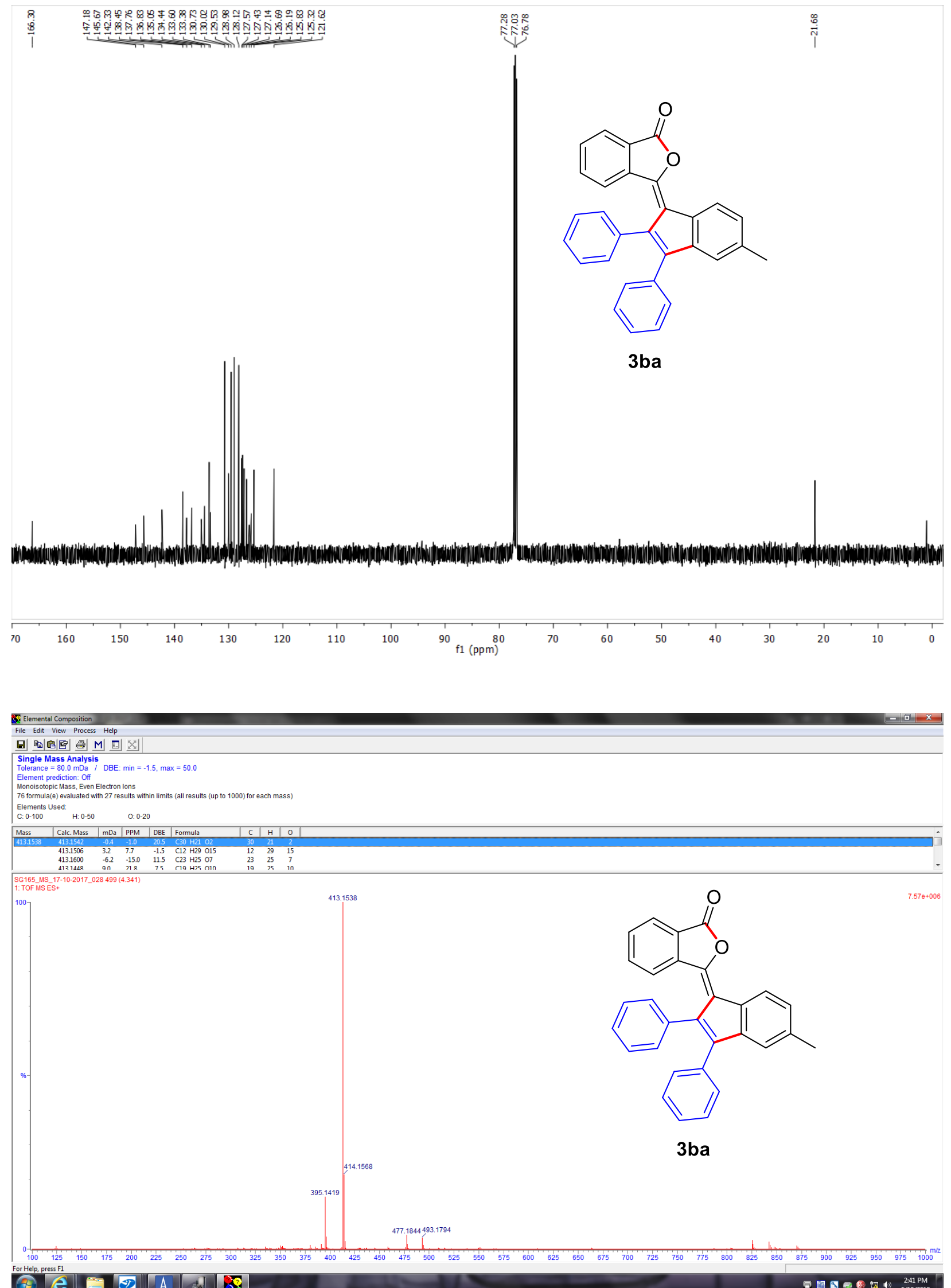


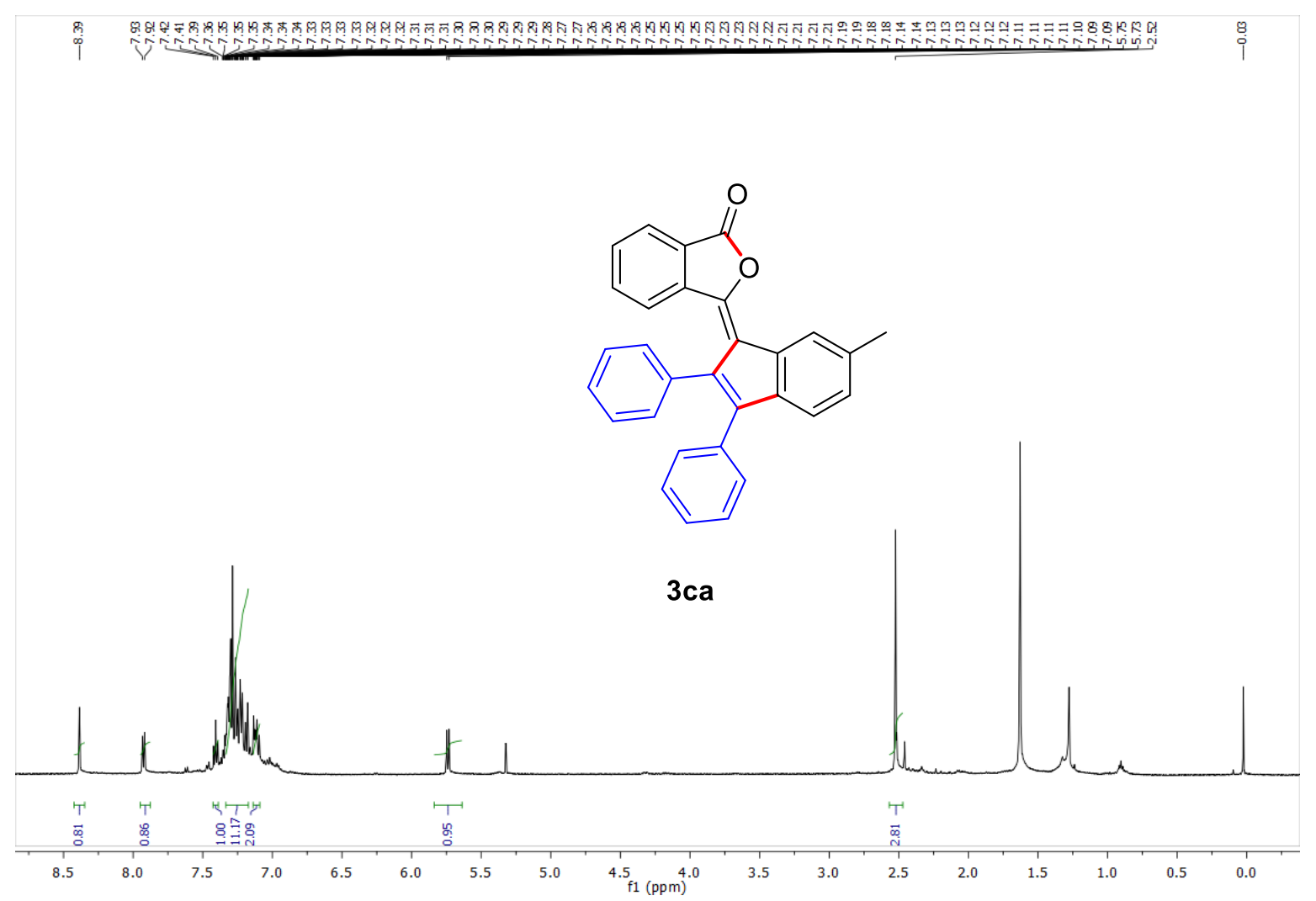

Expanded form of 3ca:

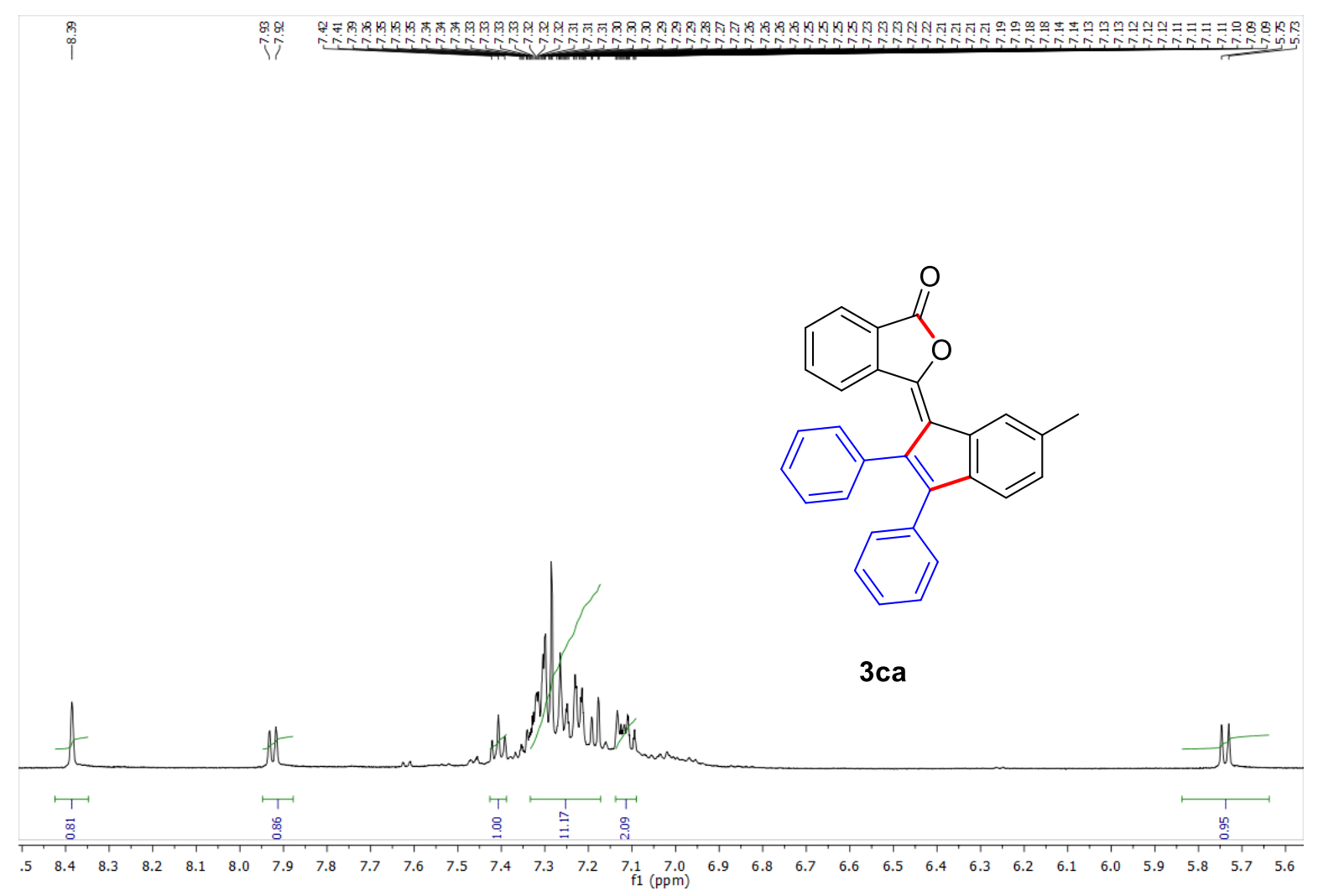




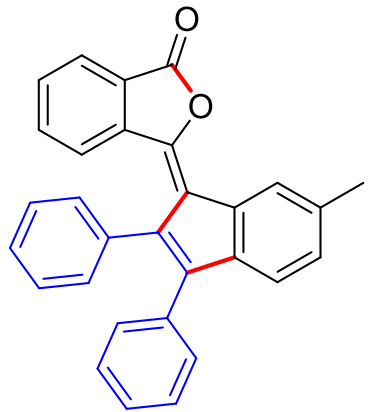

$3 c a$

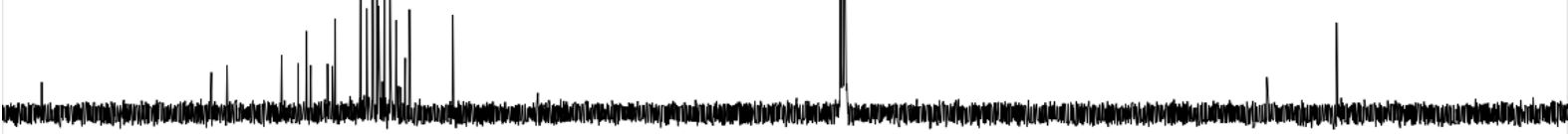
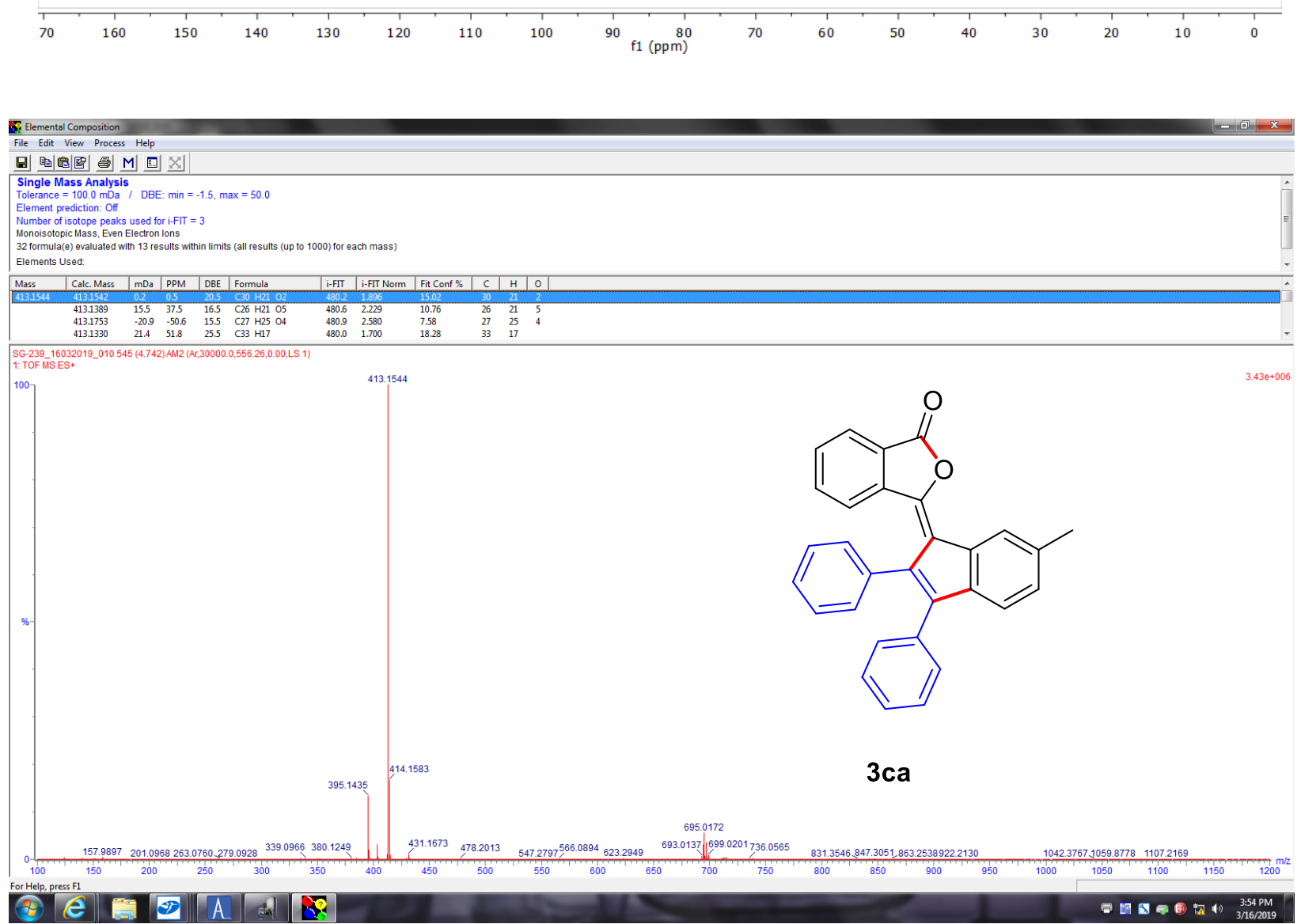


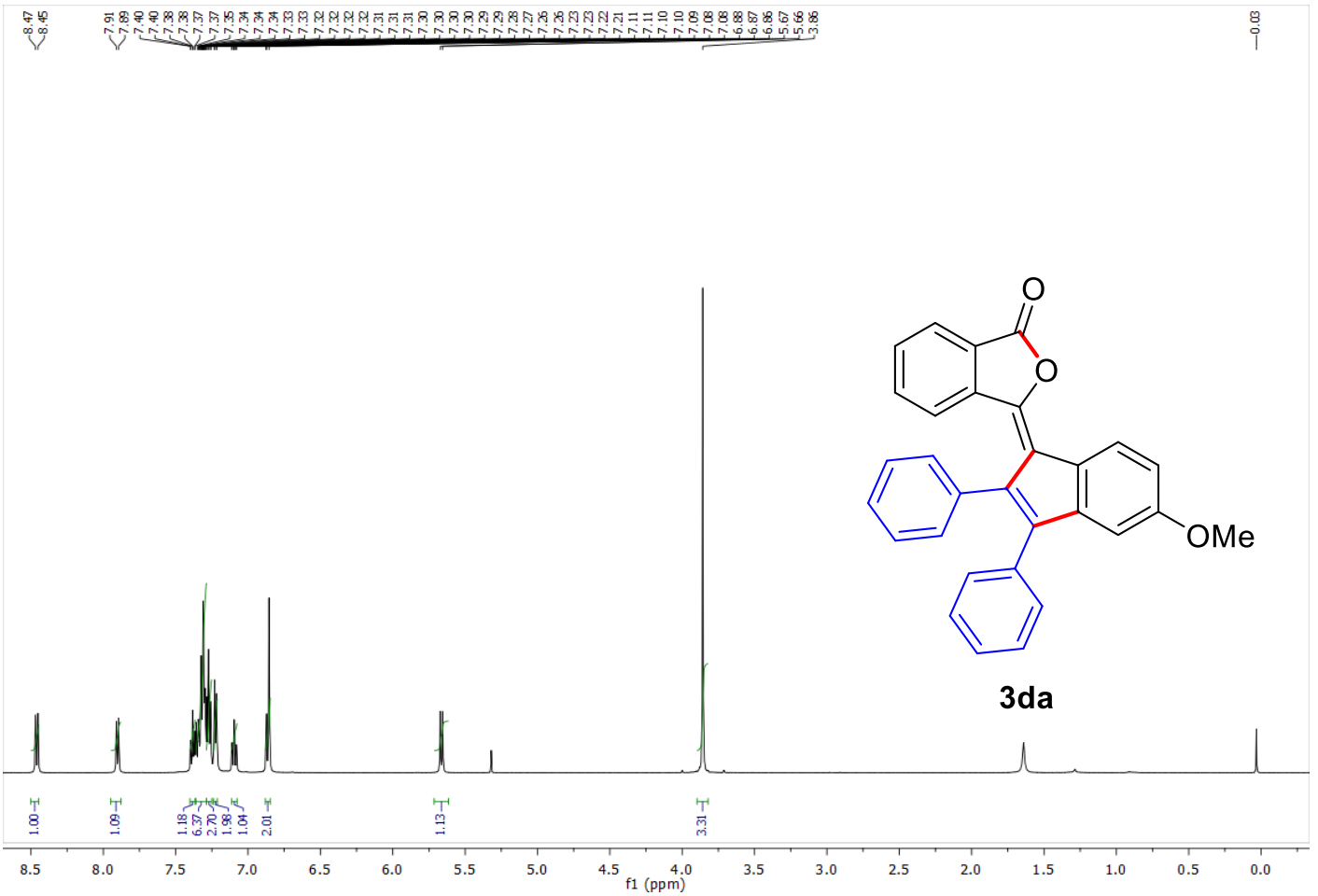

Expanded form of 3da:

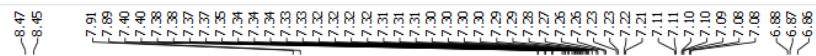

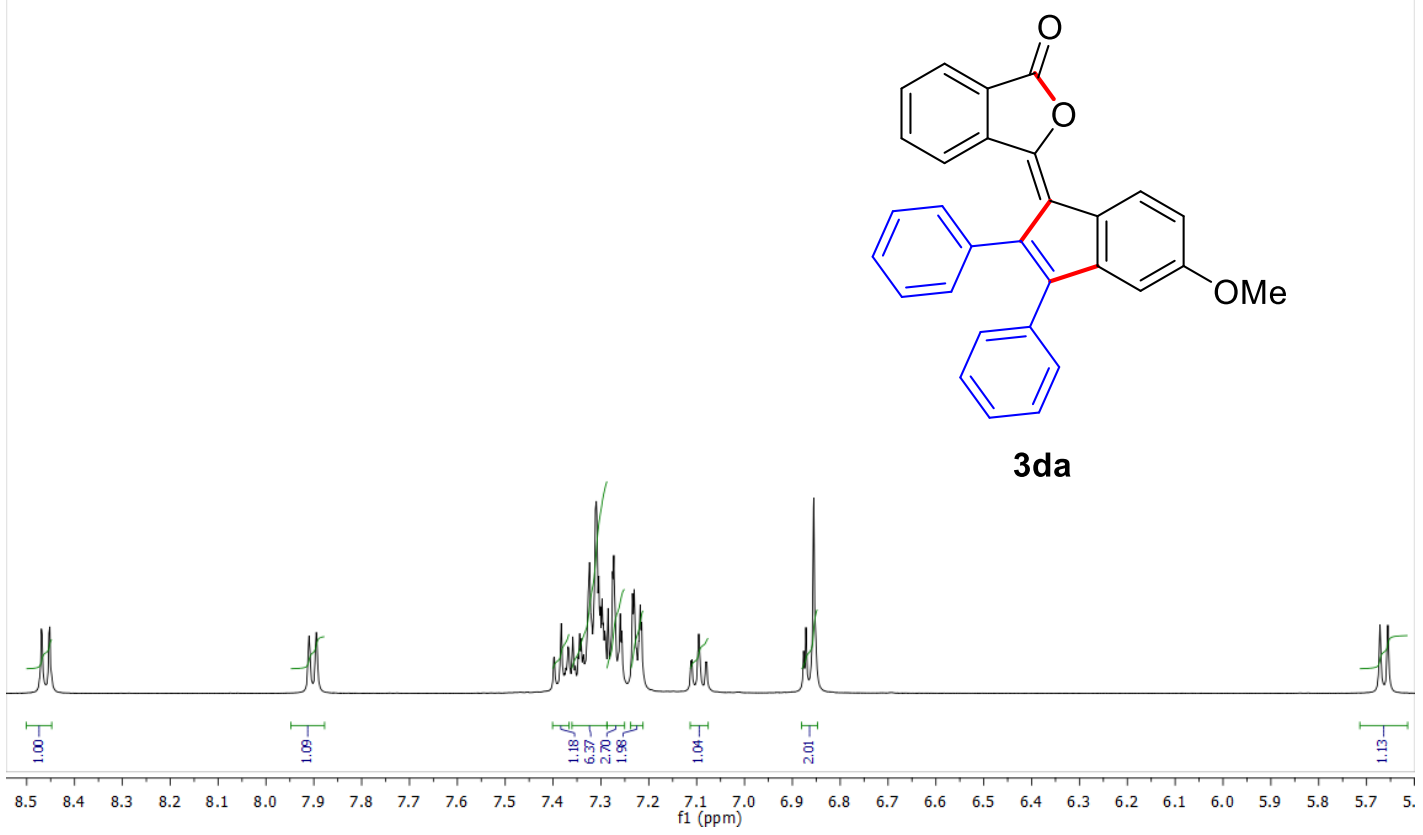



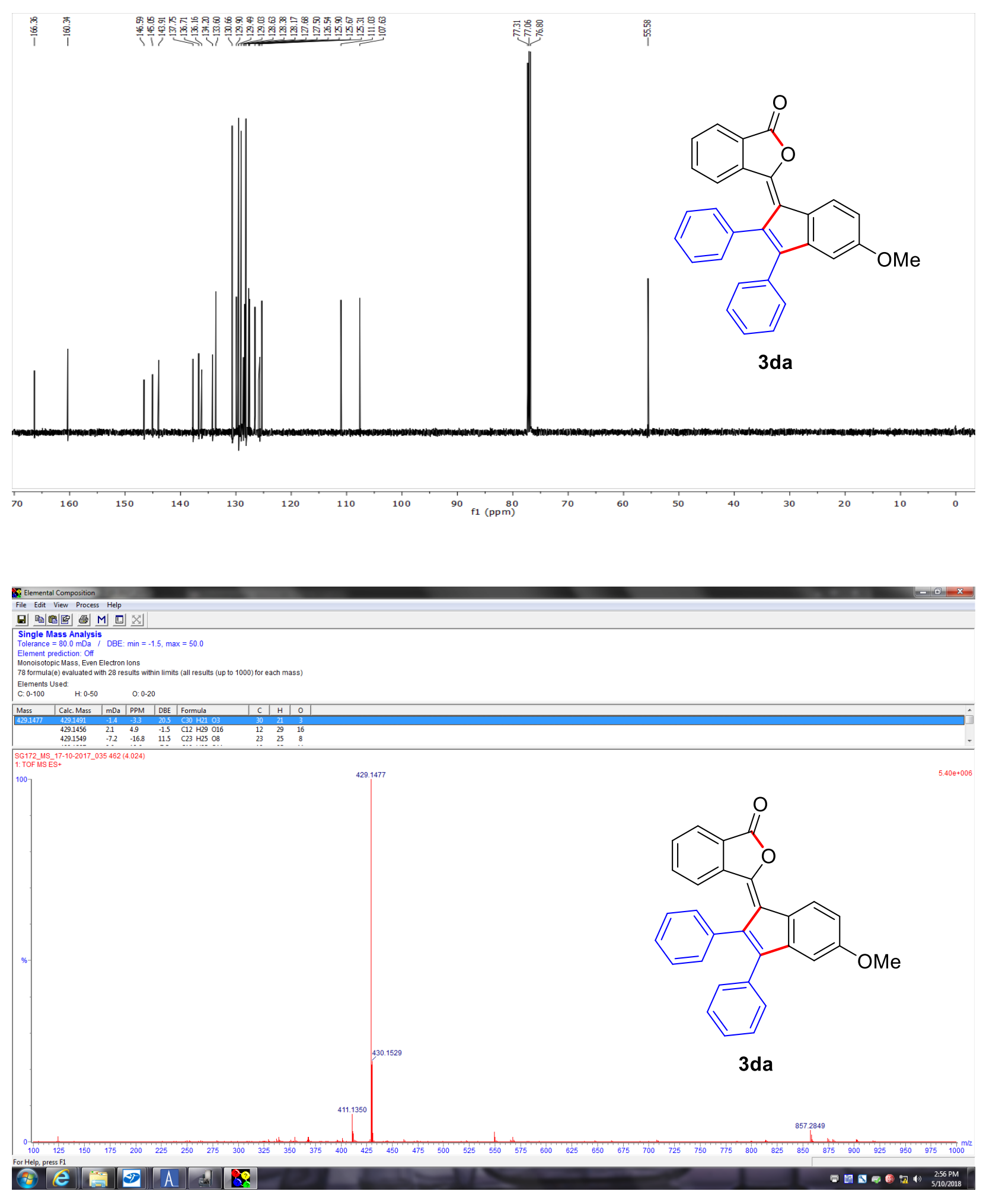


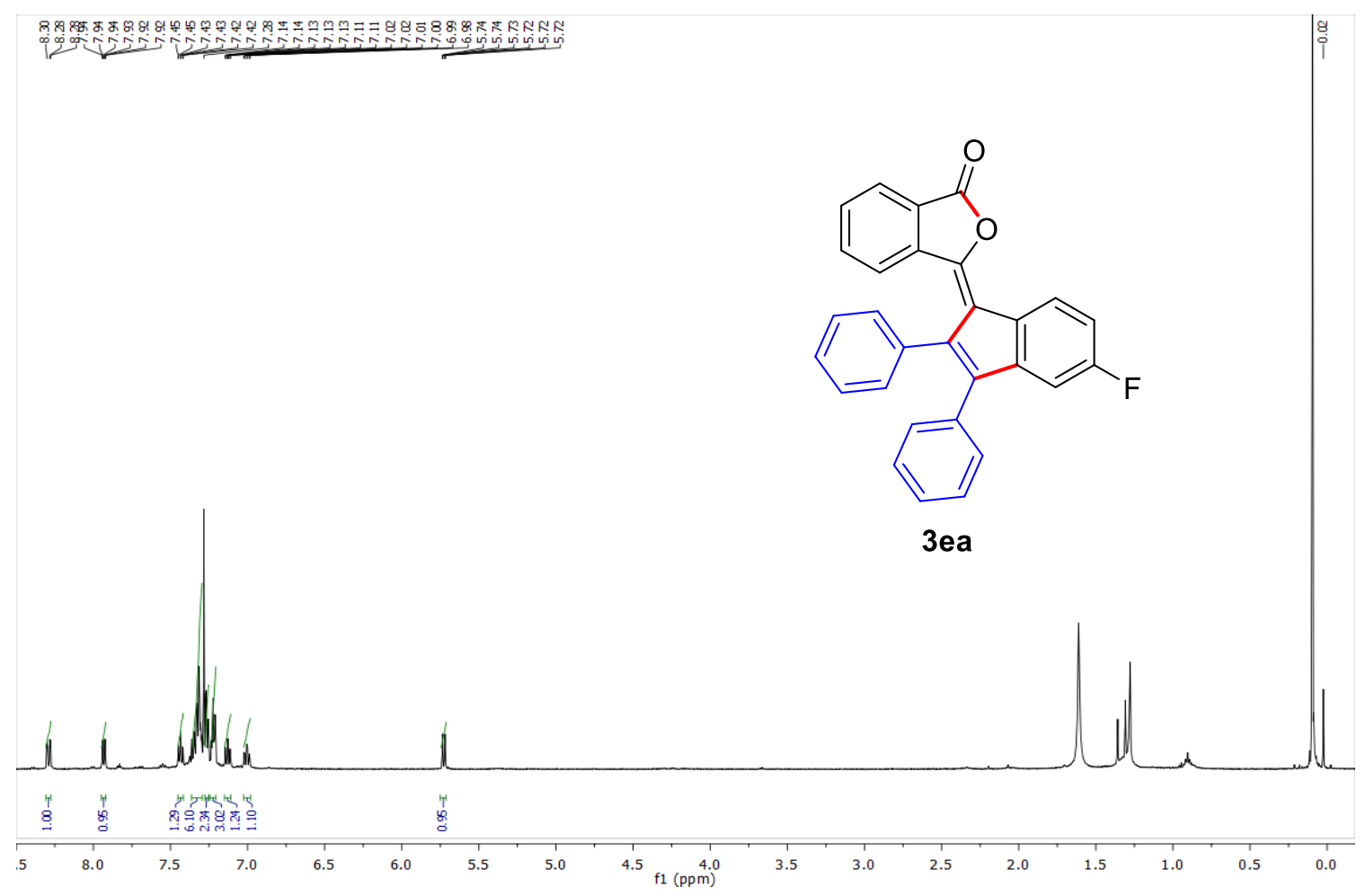

Expanded form of 3ea:

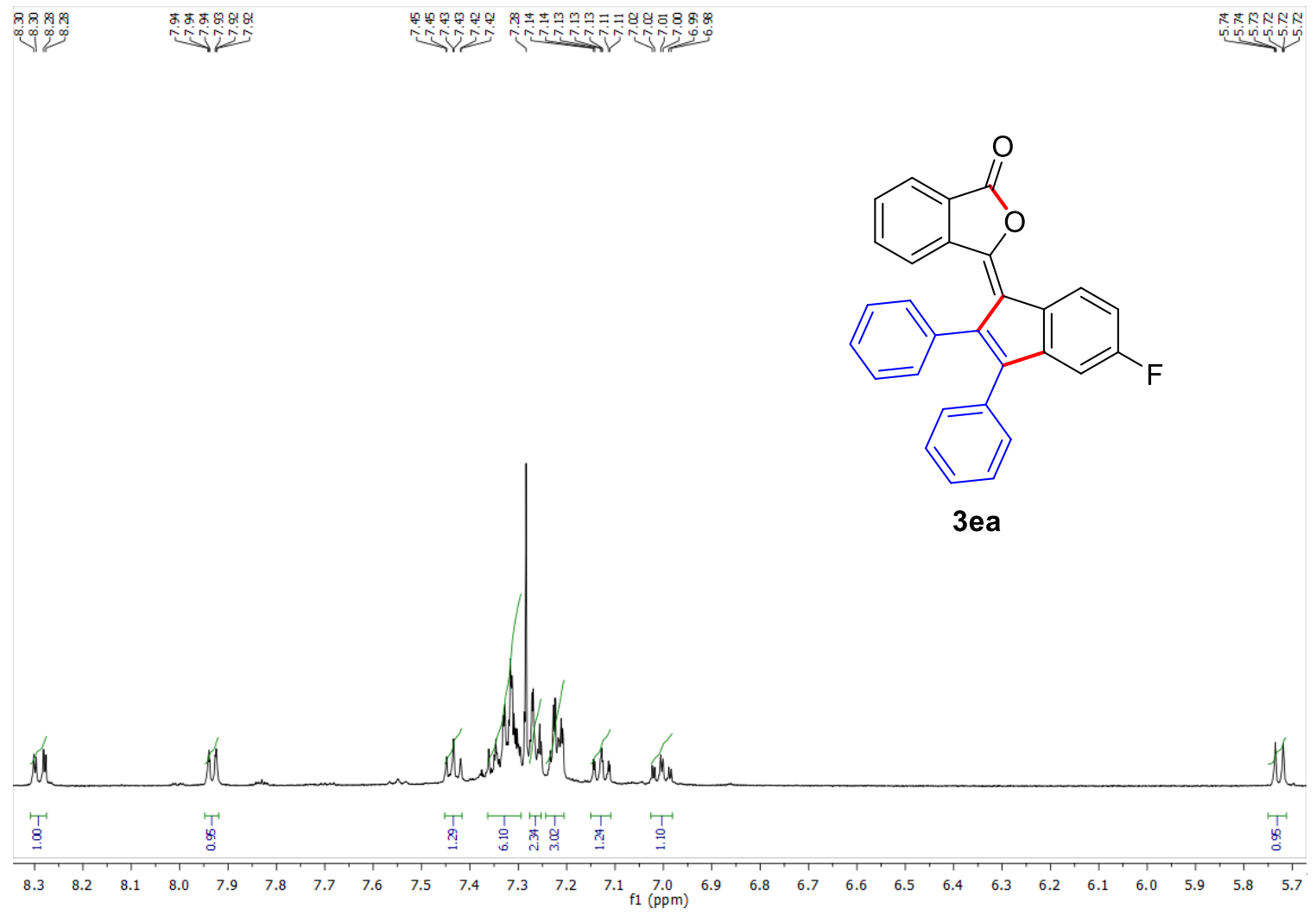



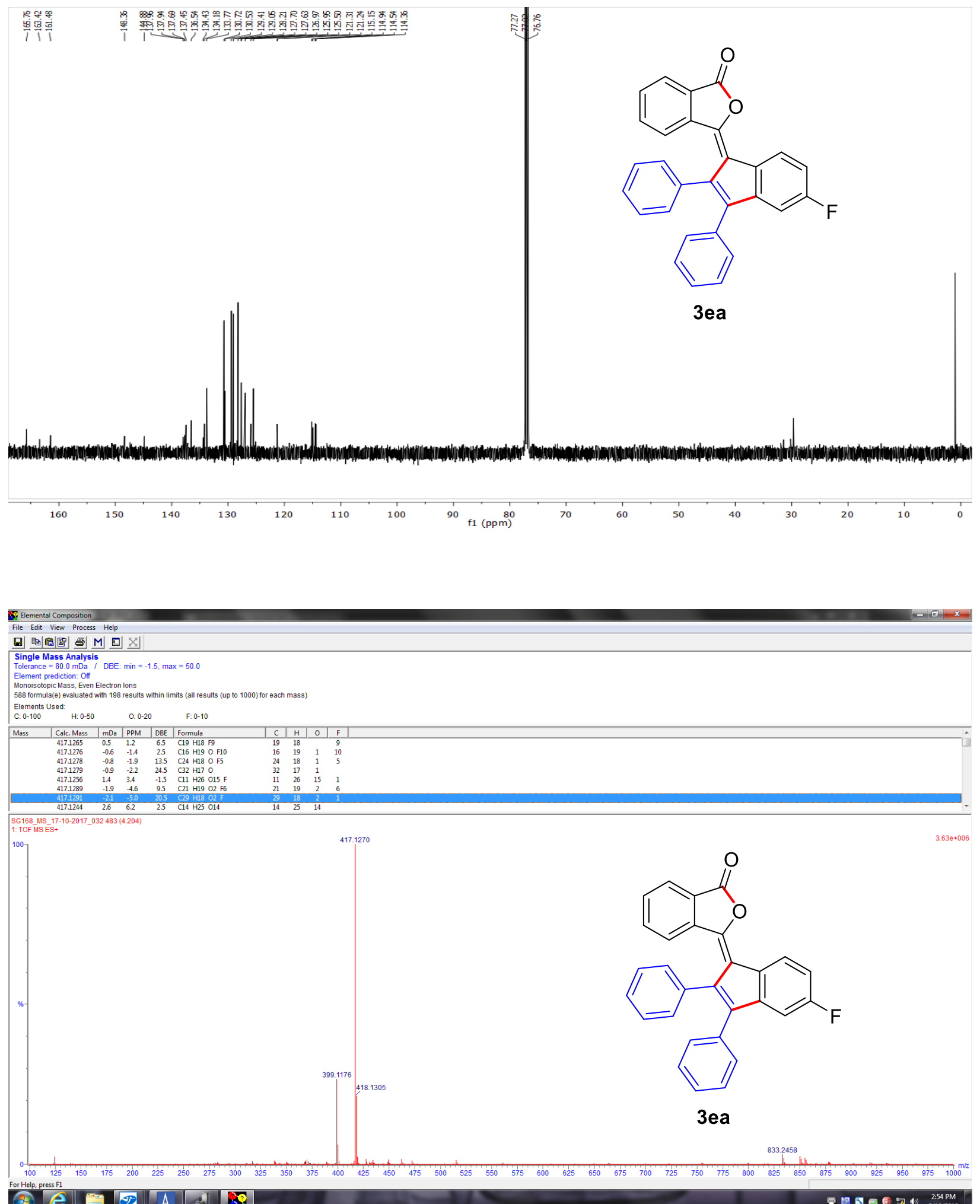


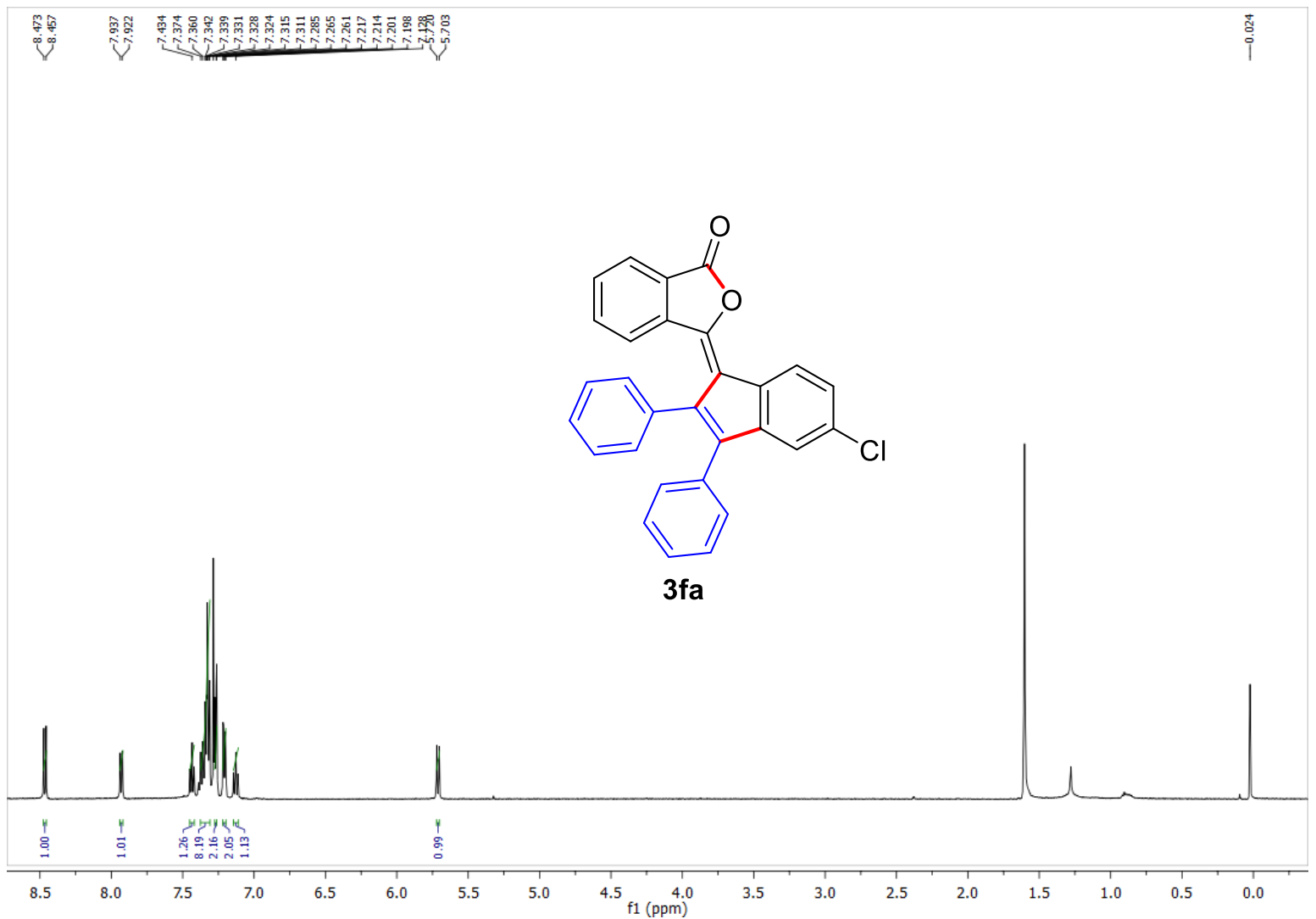

Expanded form of 3fa:

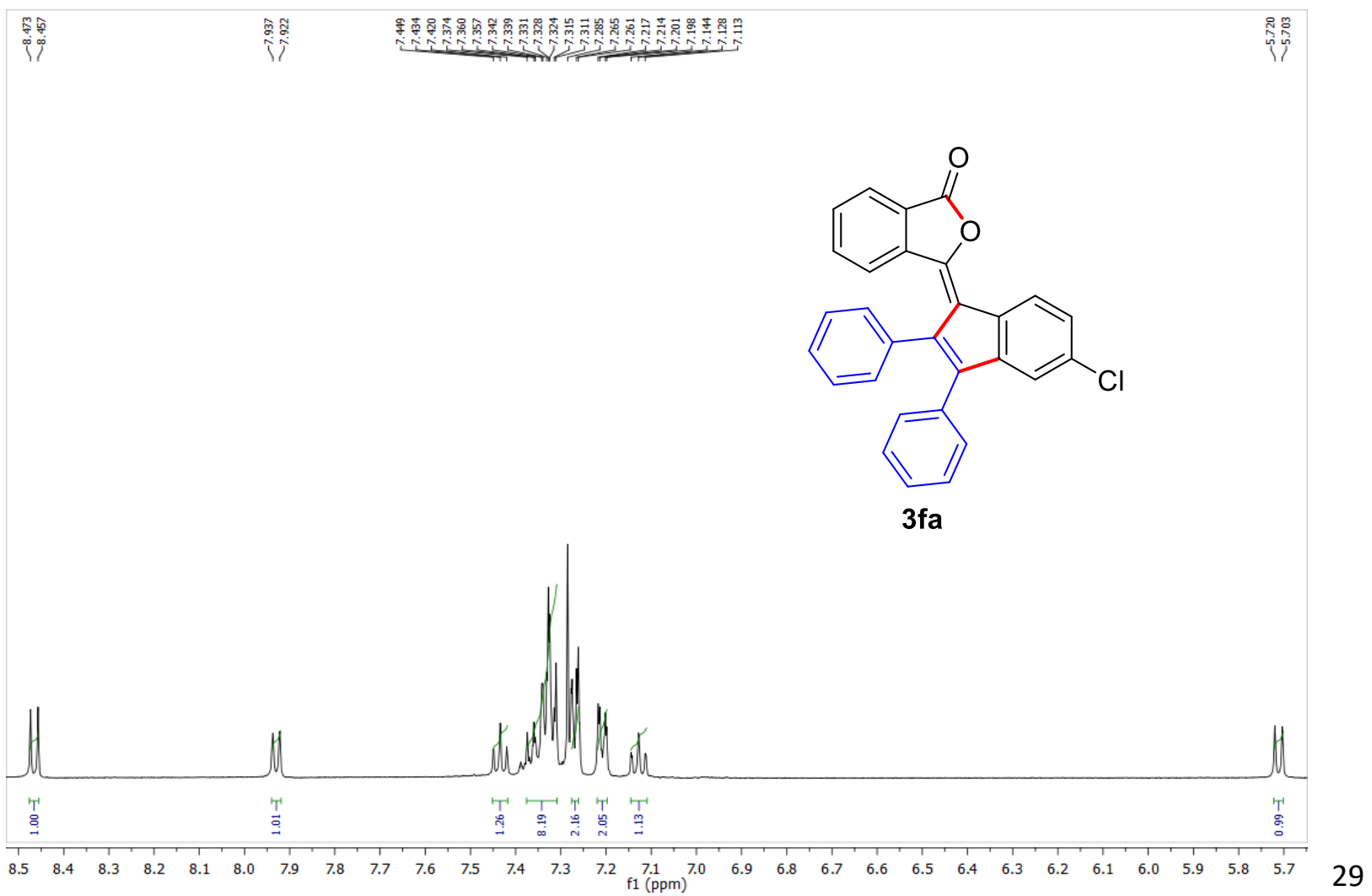



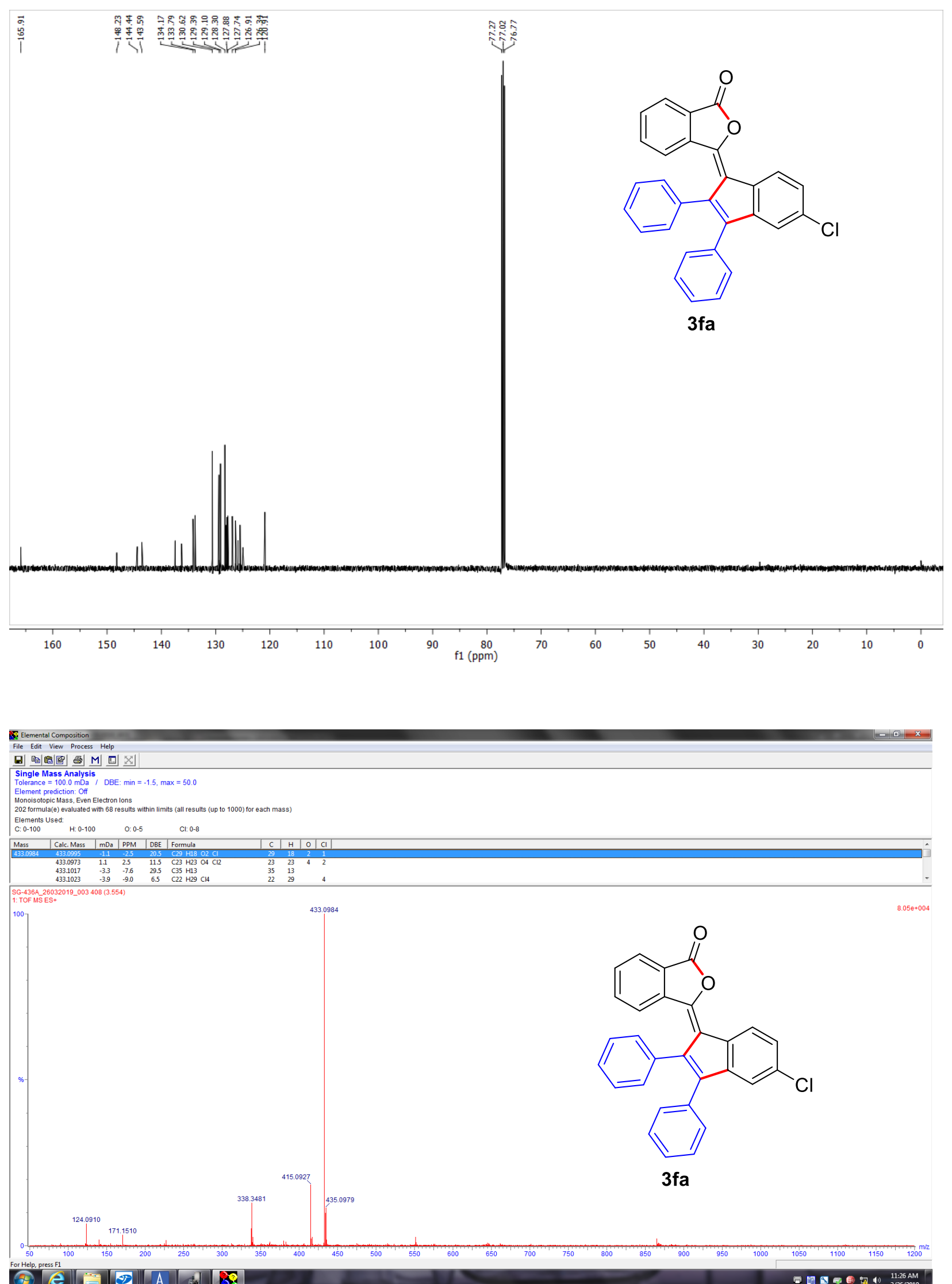


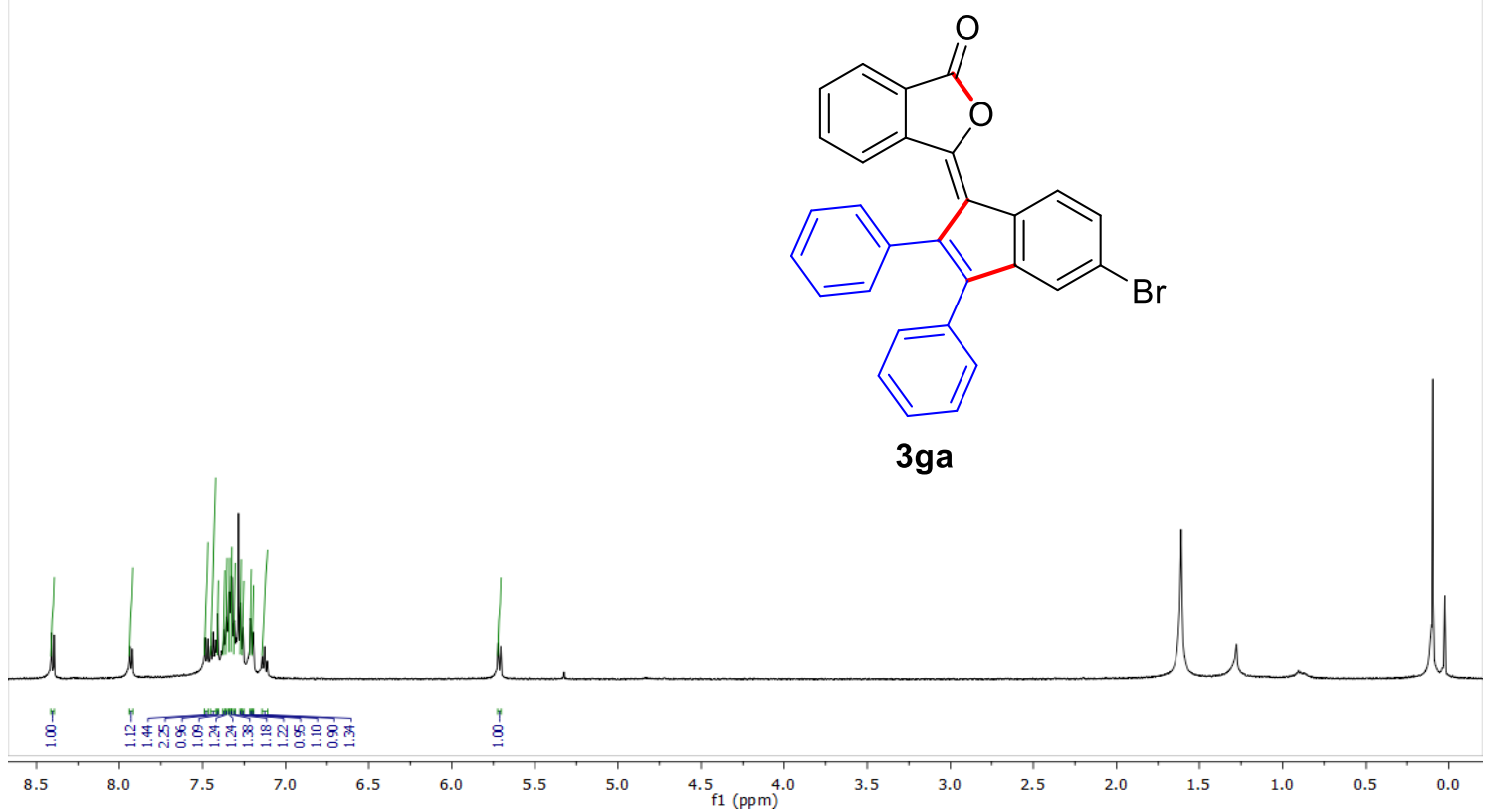

Expanded form of 3ga:

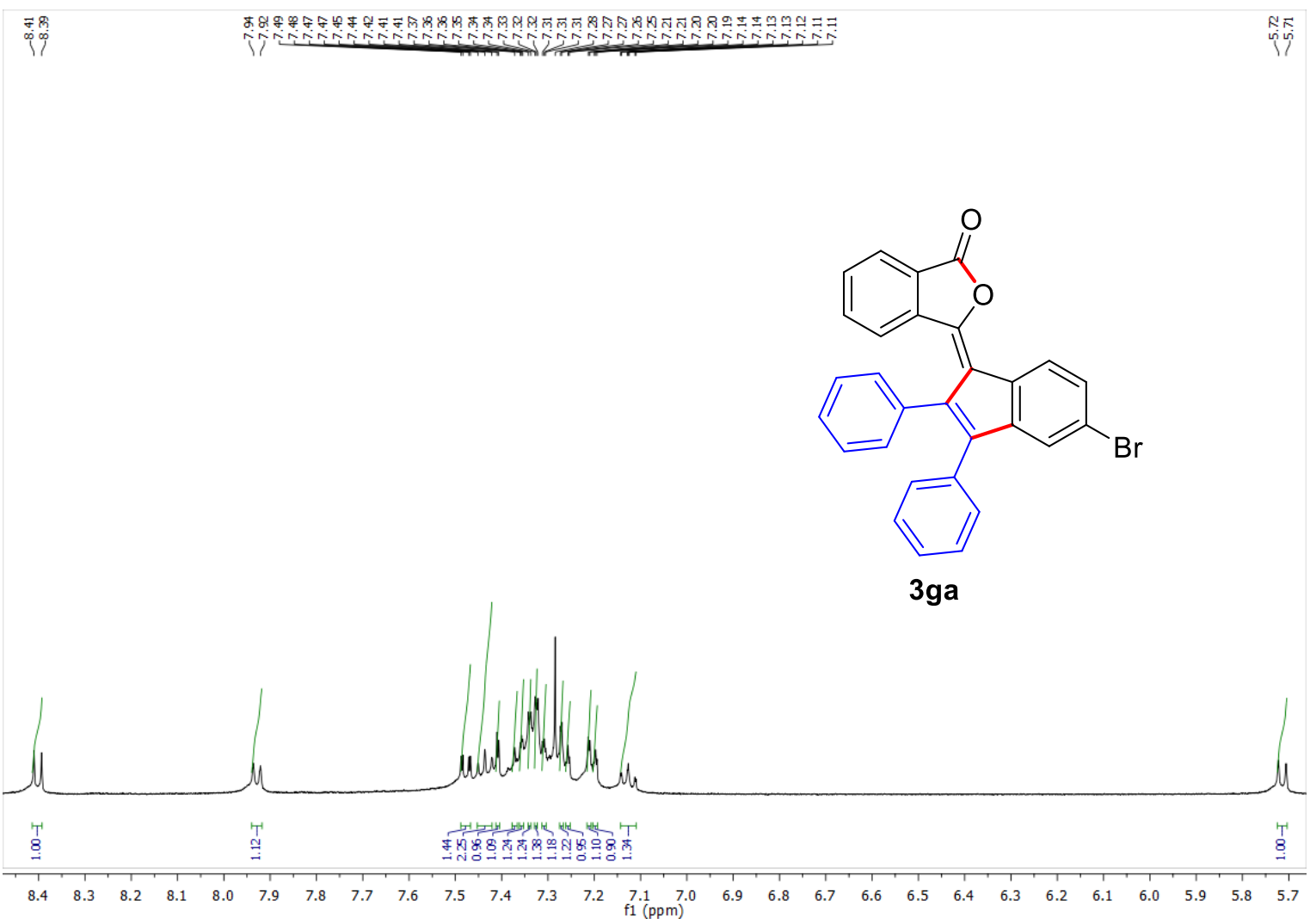



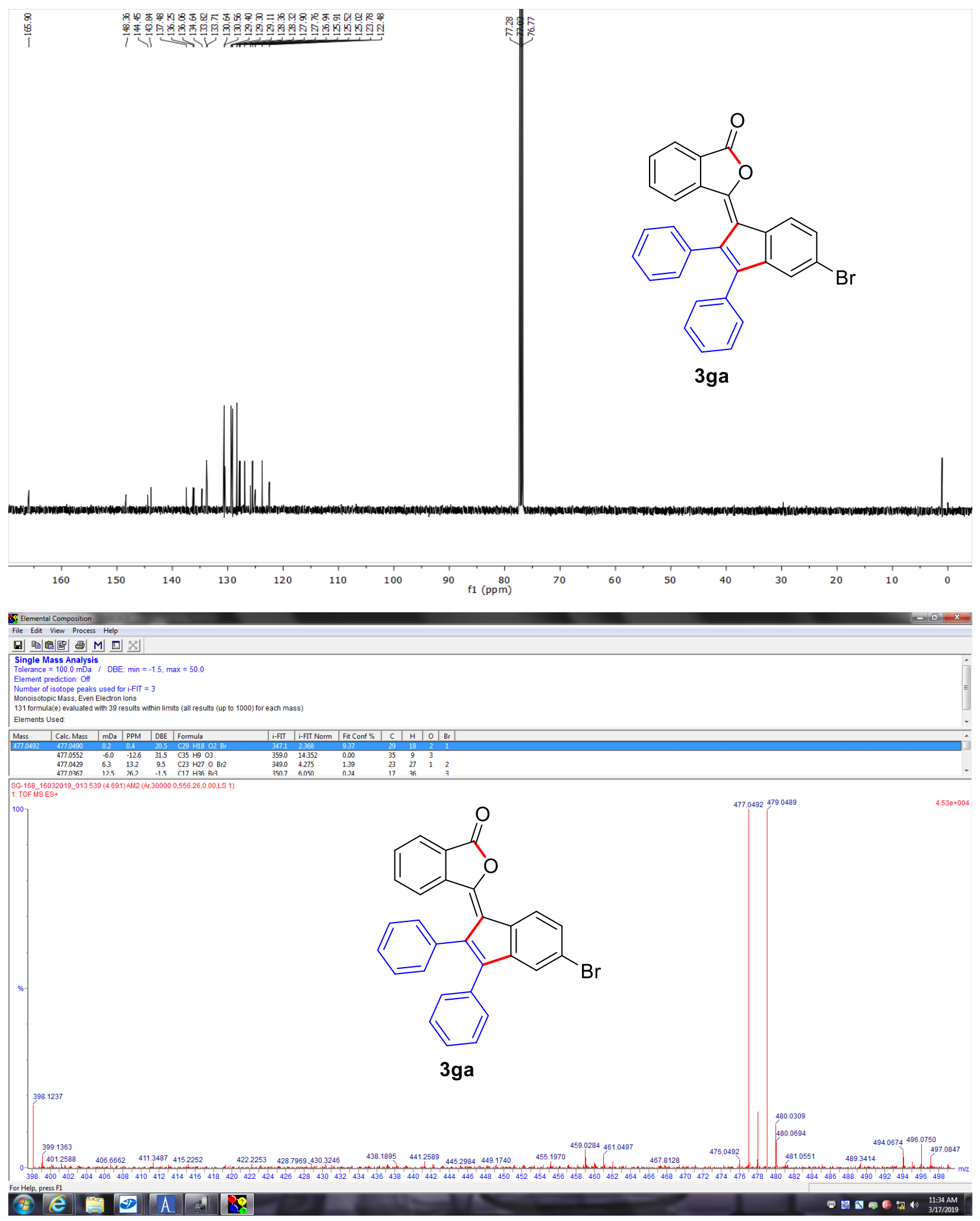


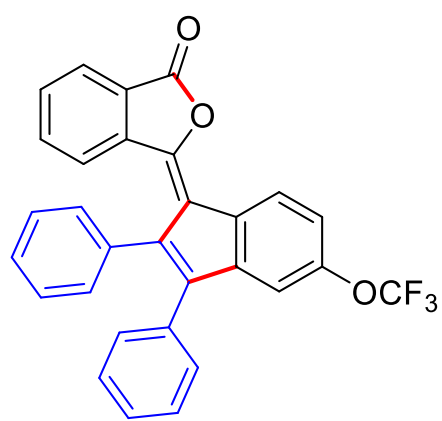

3ha

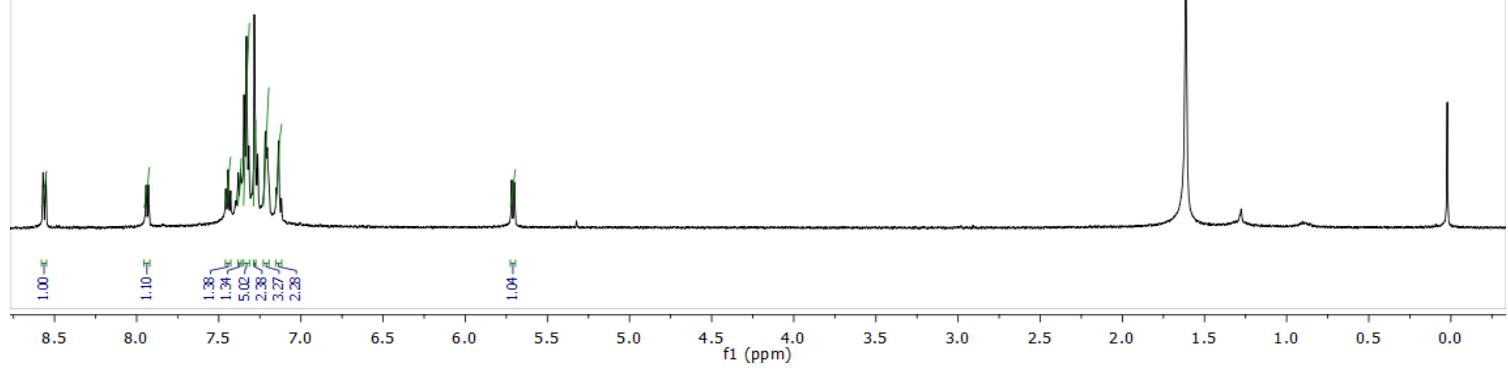

Expanded form of 3ha:

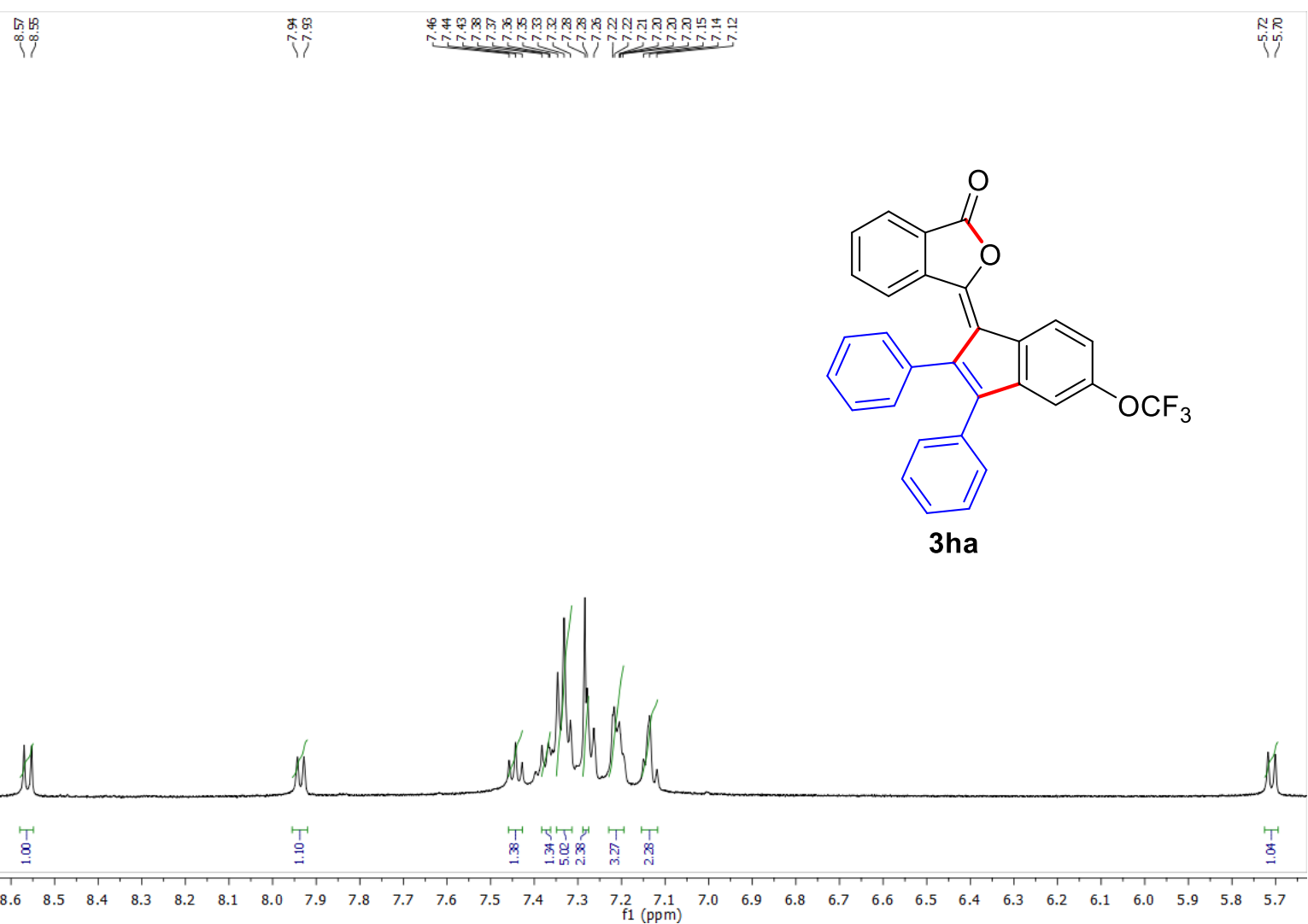



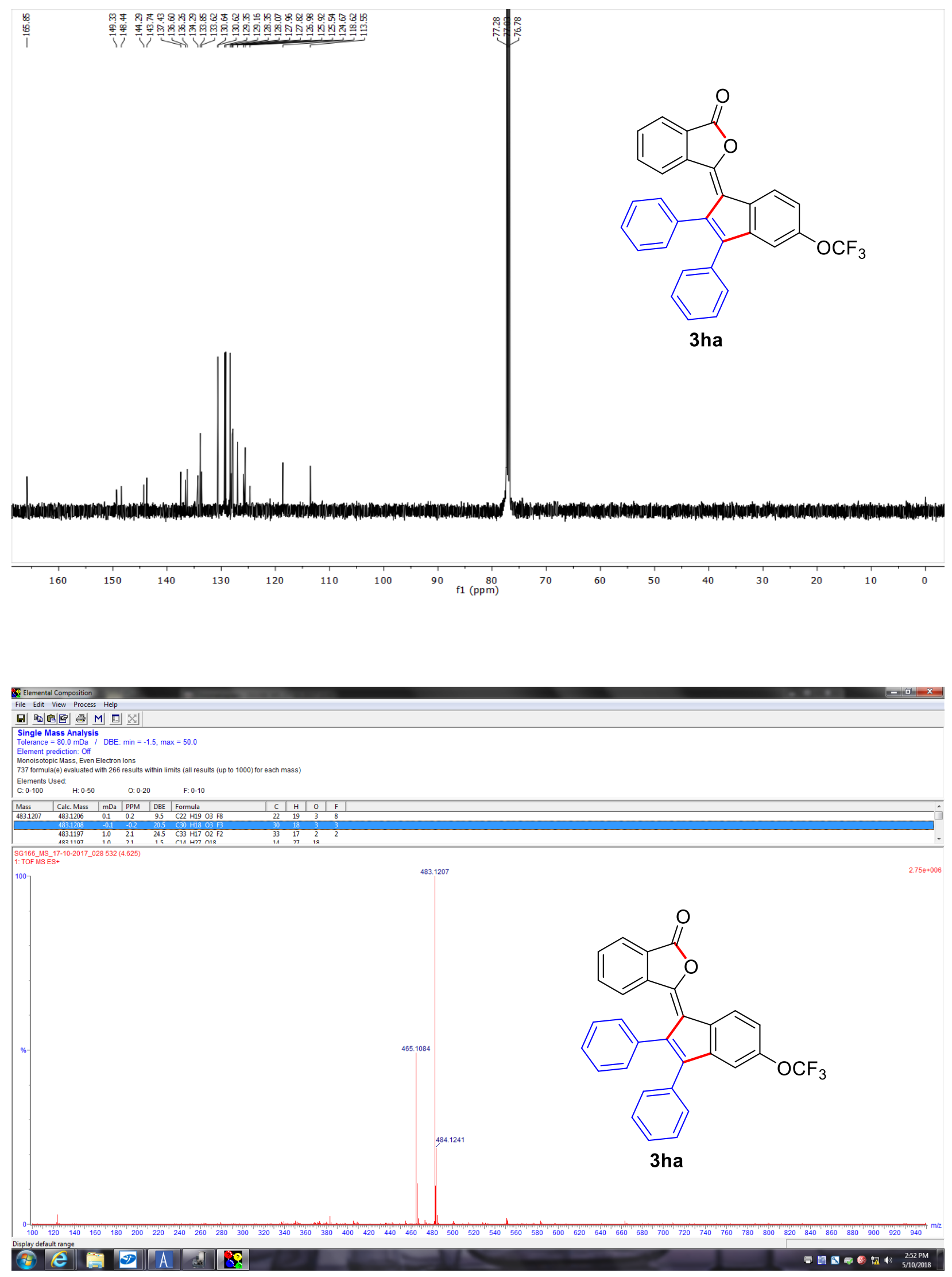


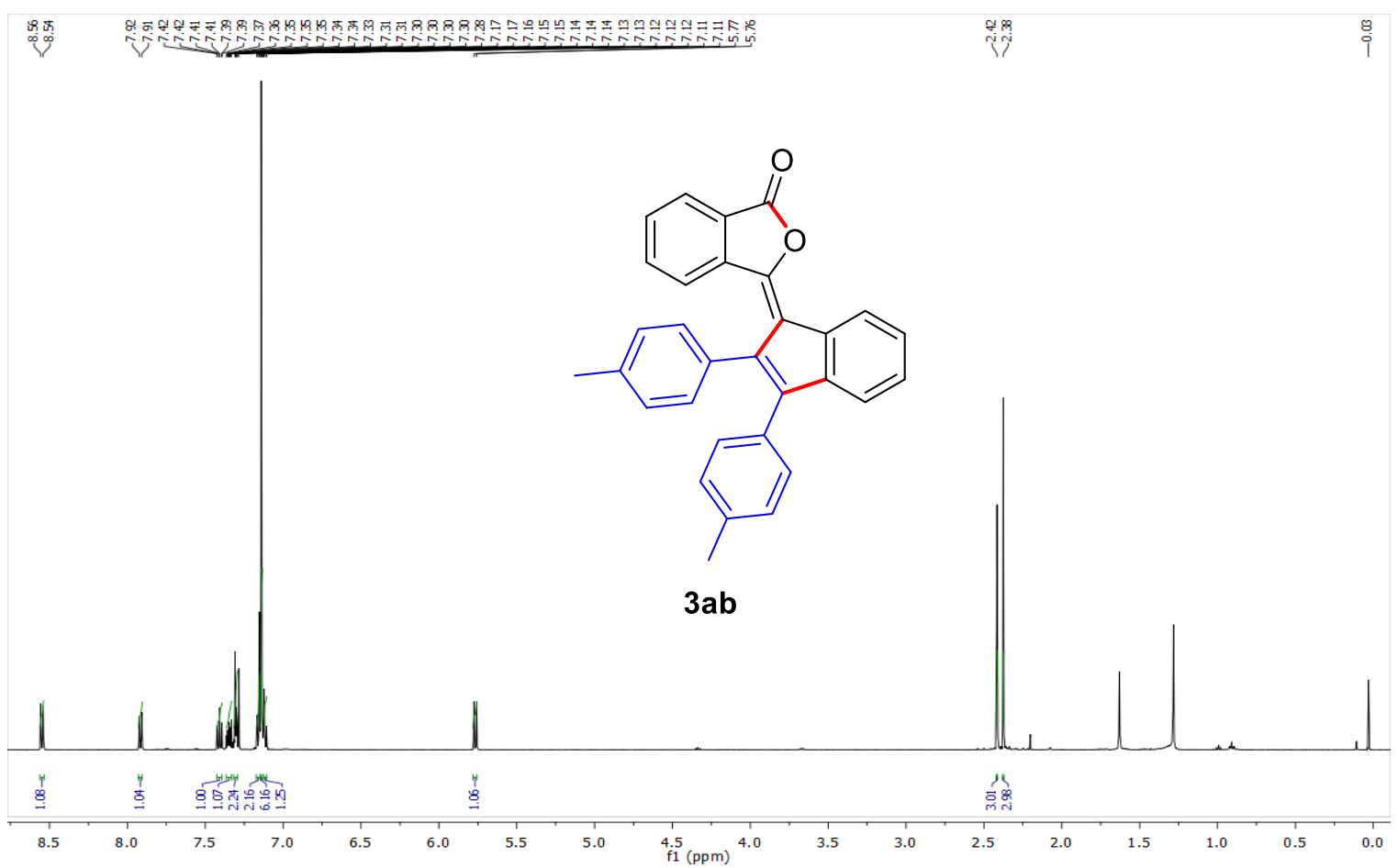

Expanded form of $3 \mathrm{ab}$ :

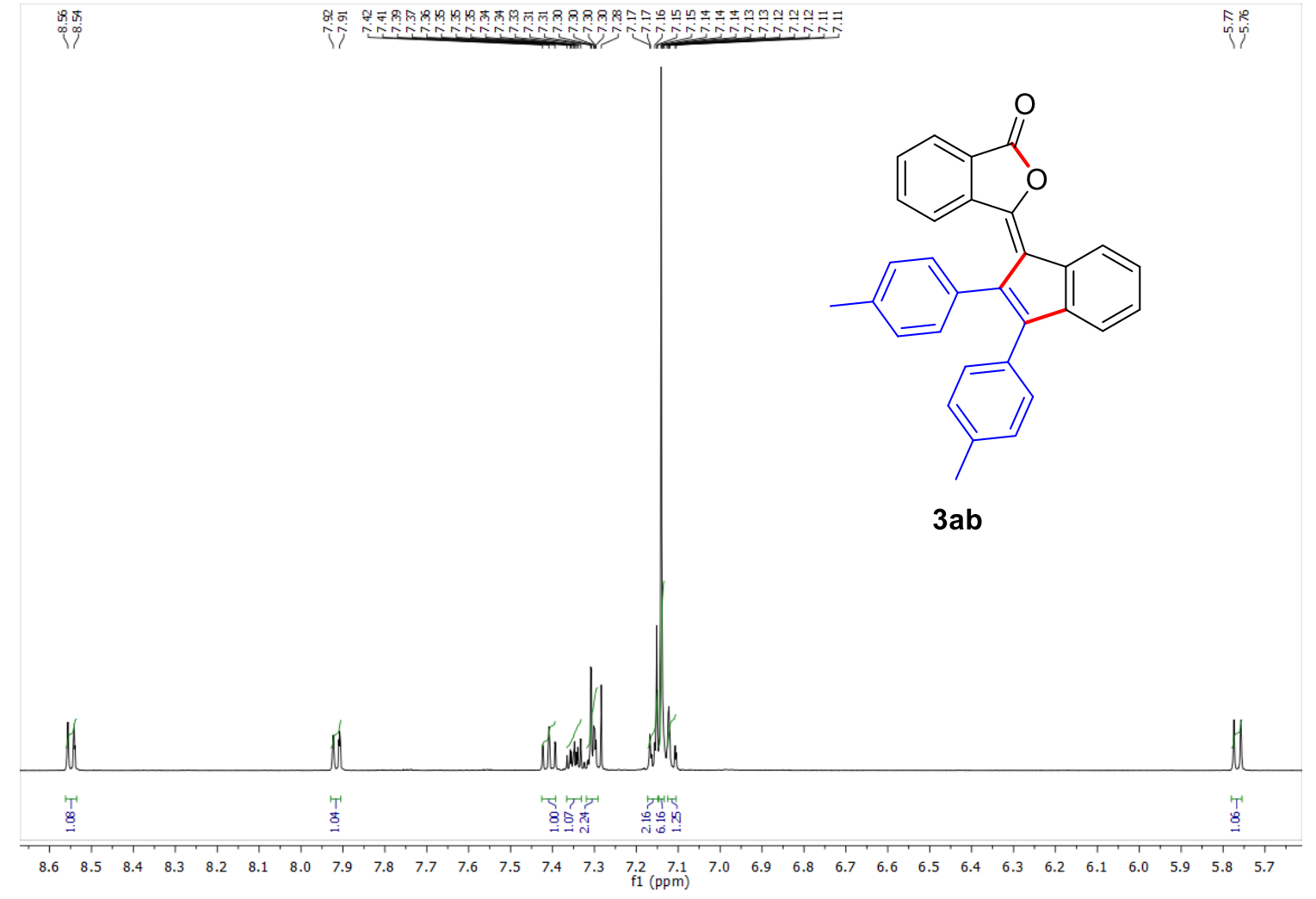




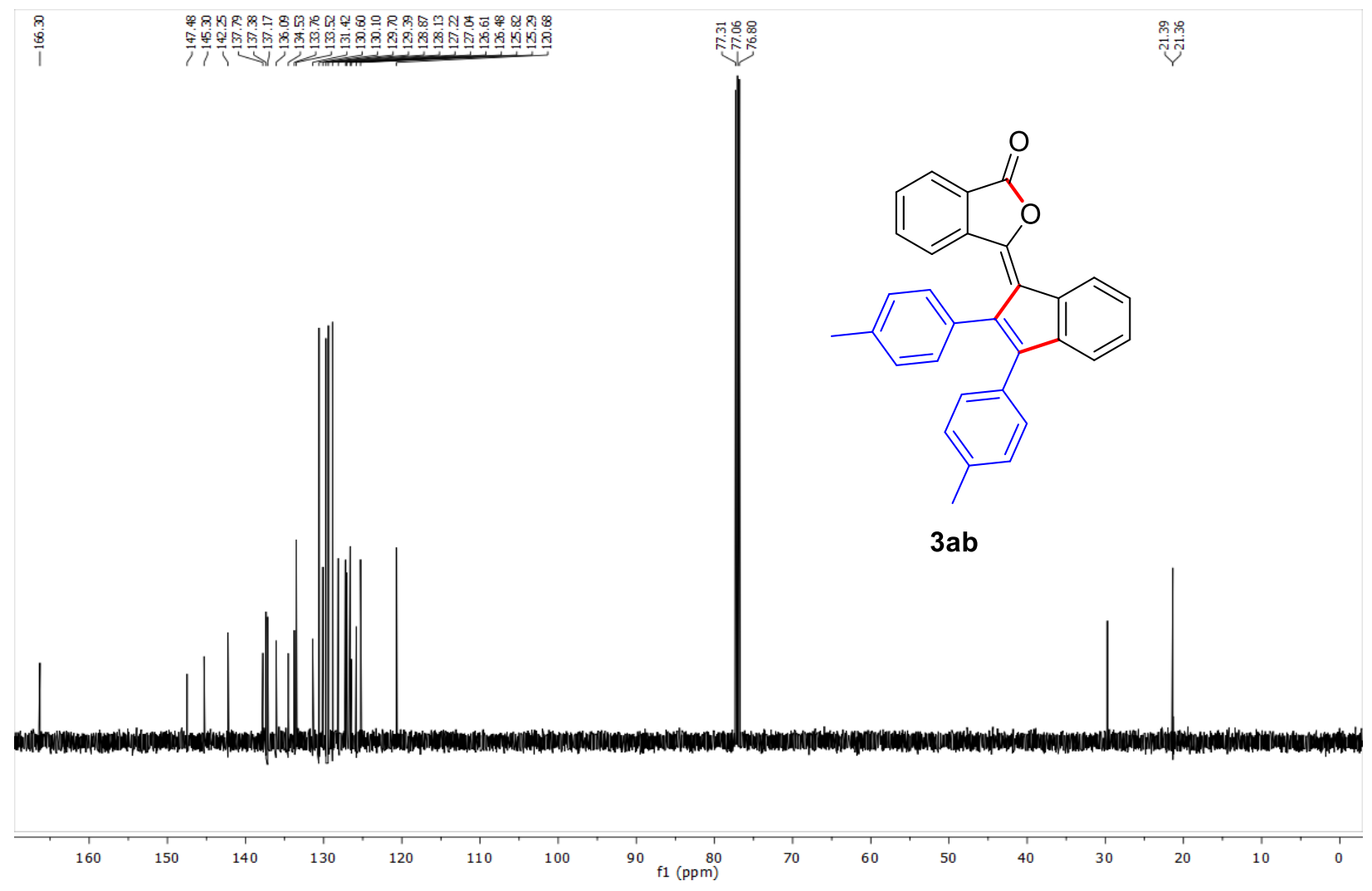




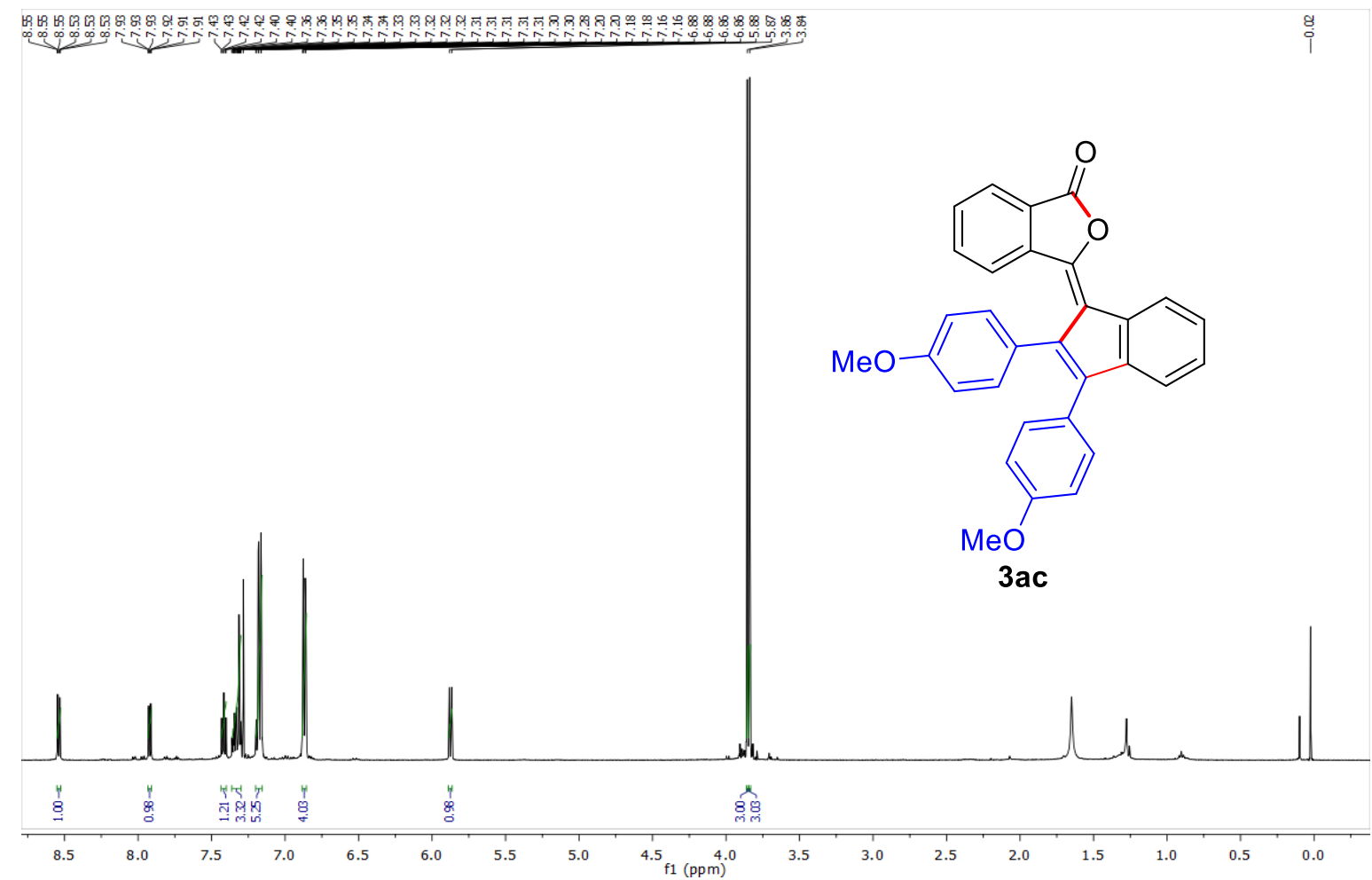

Expanded form of 3ac:

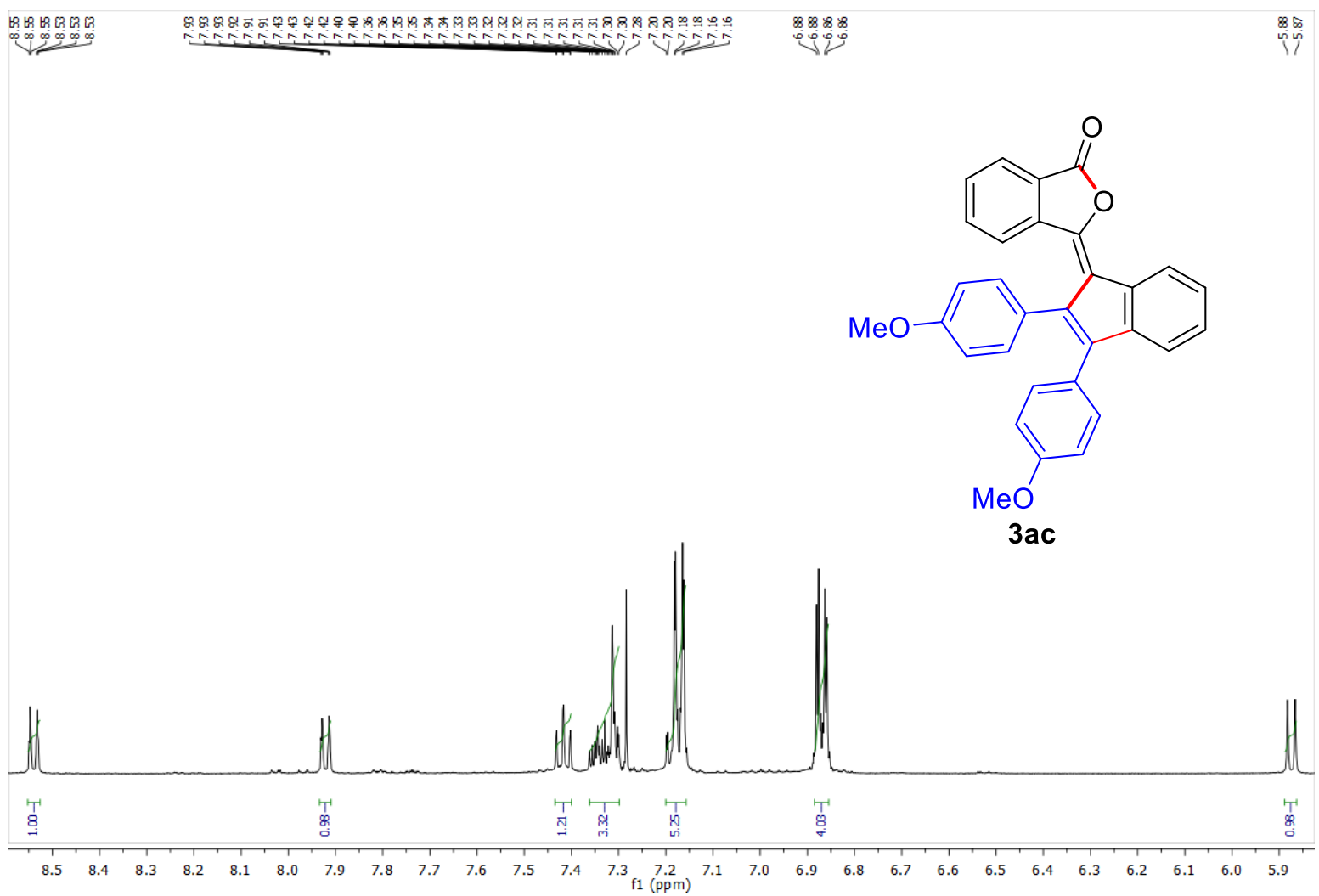



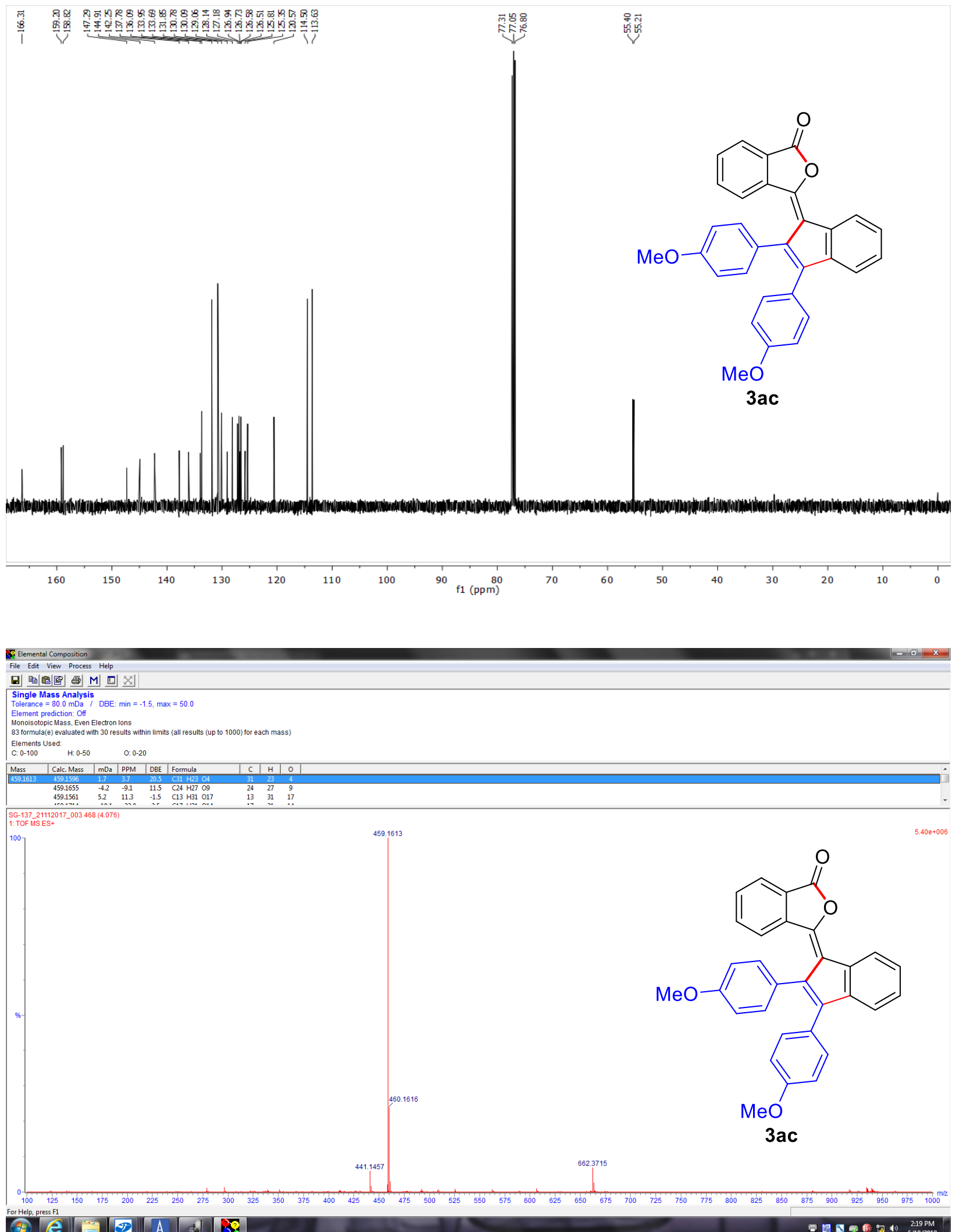


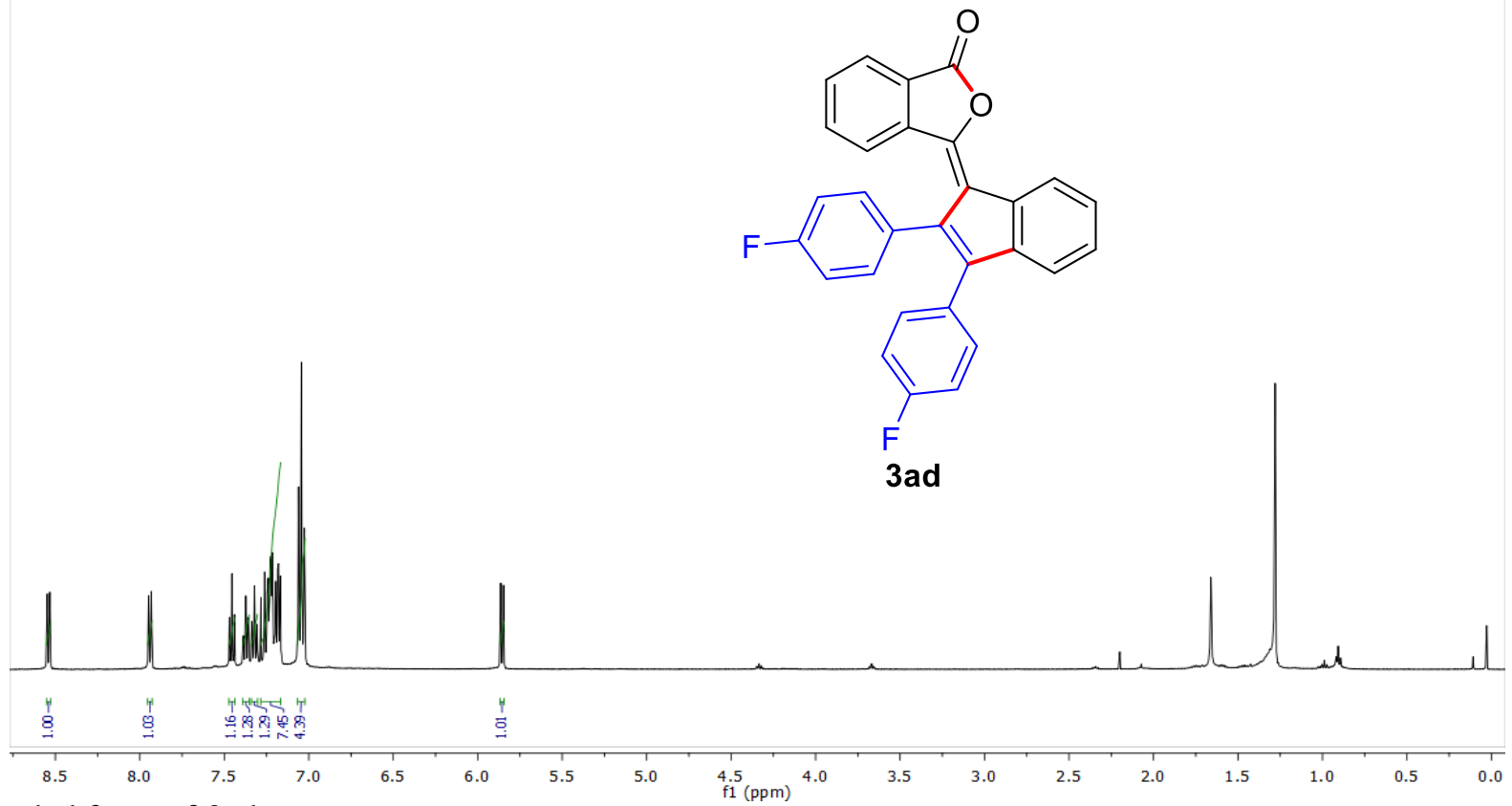

Expanded form of $3 \mathrm{ad}$ :

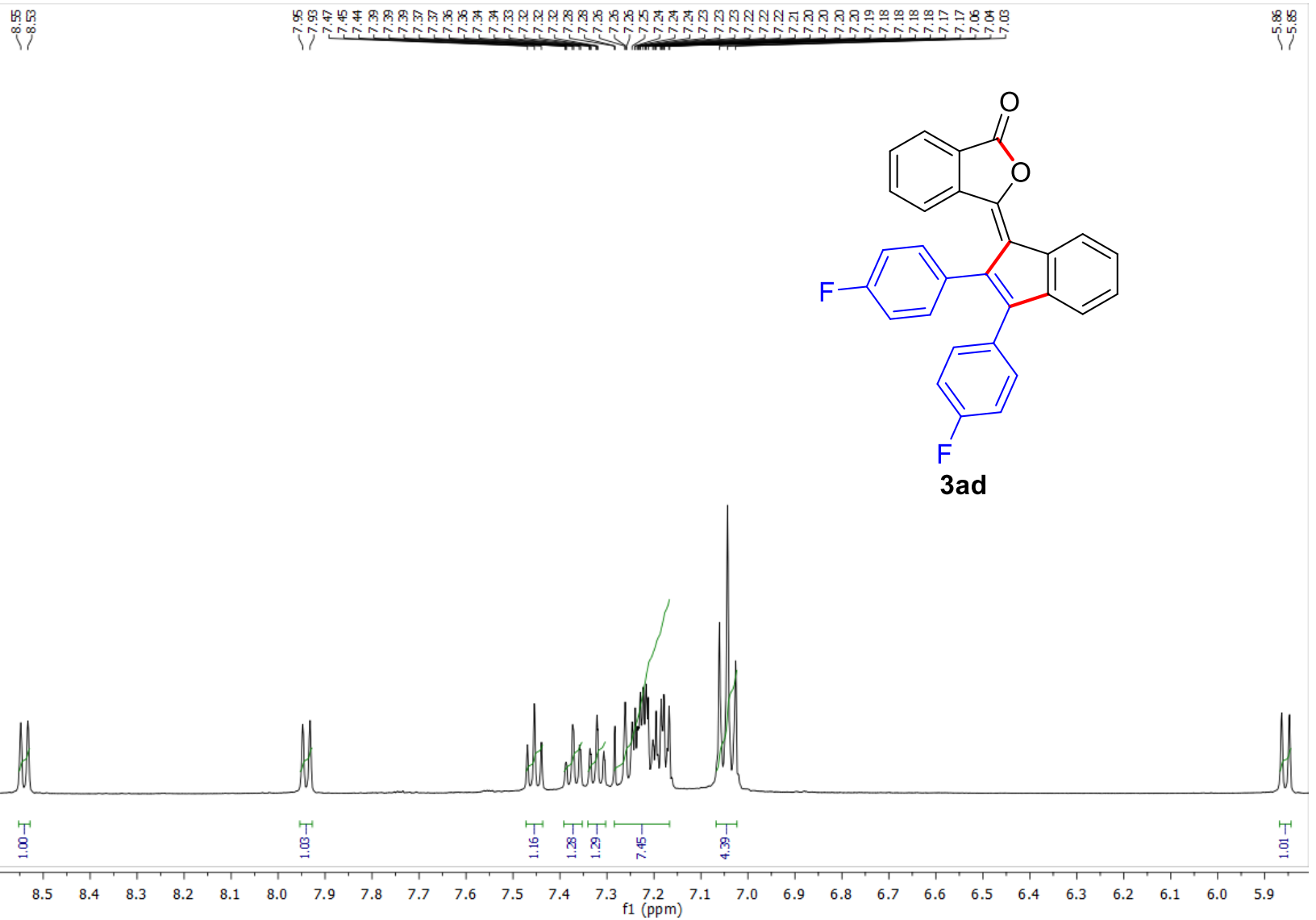




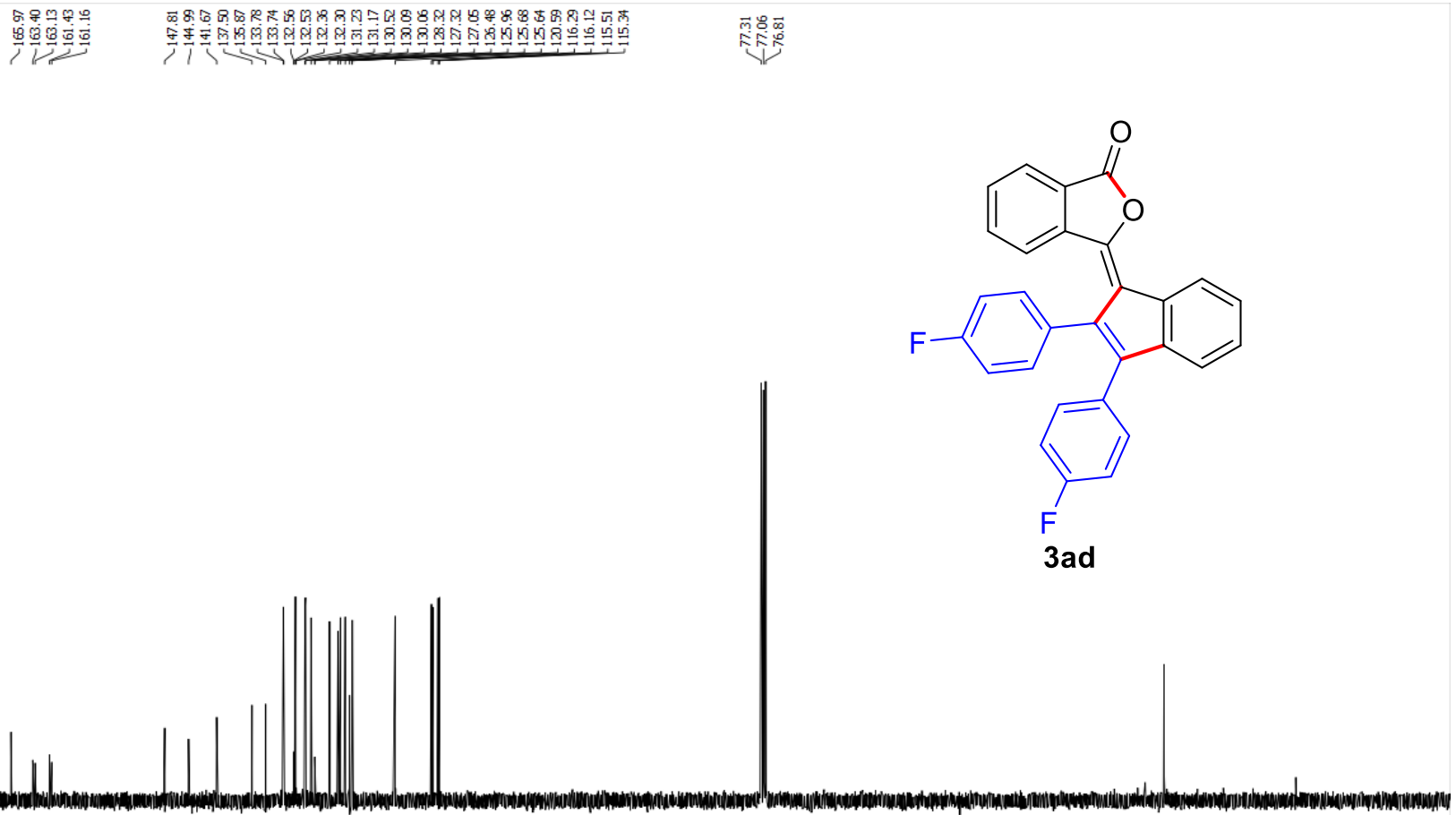

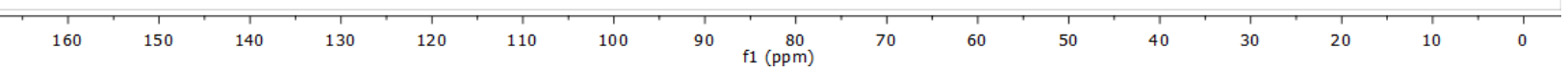

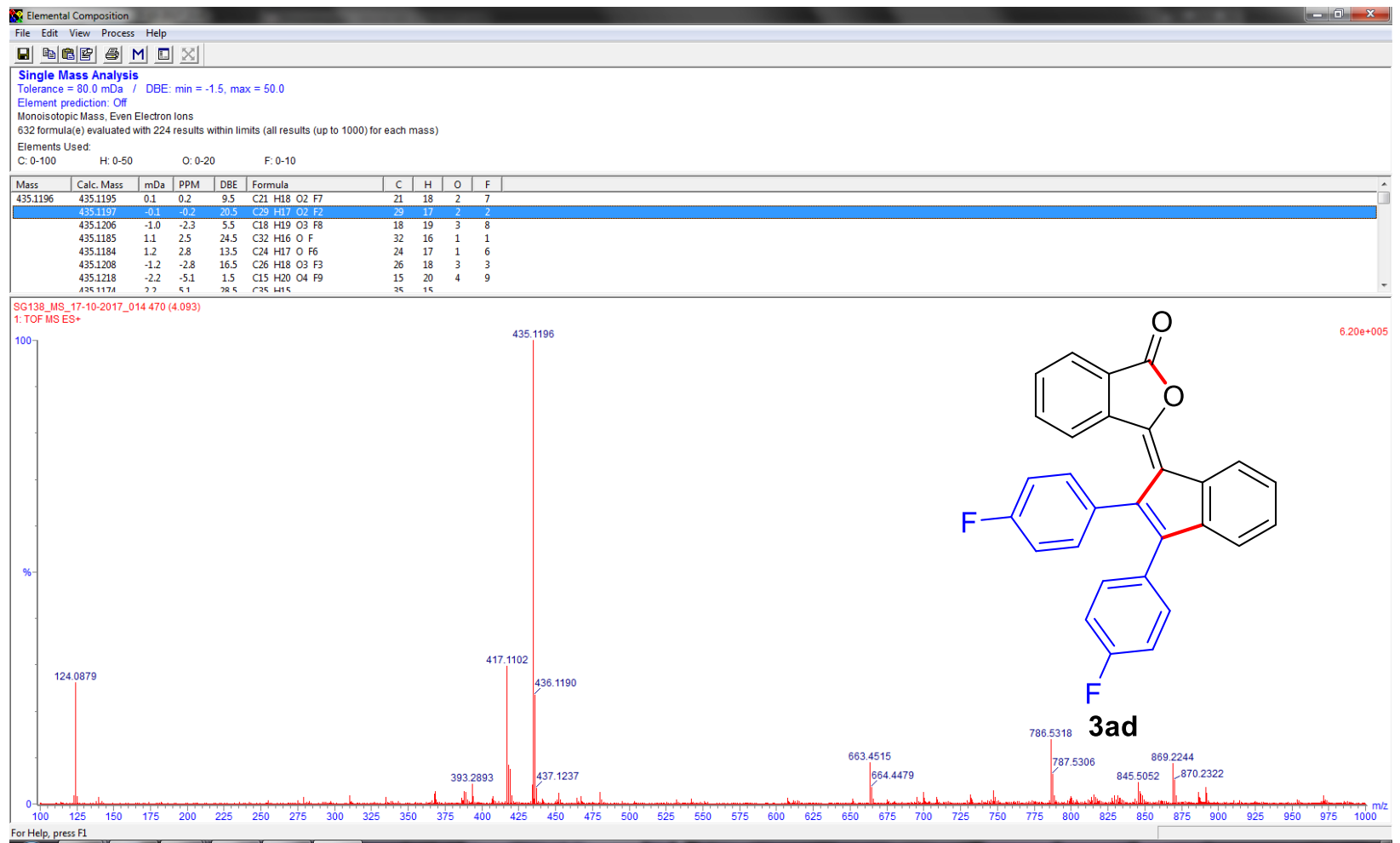




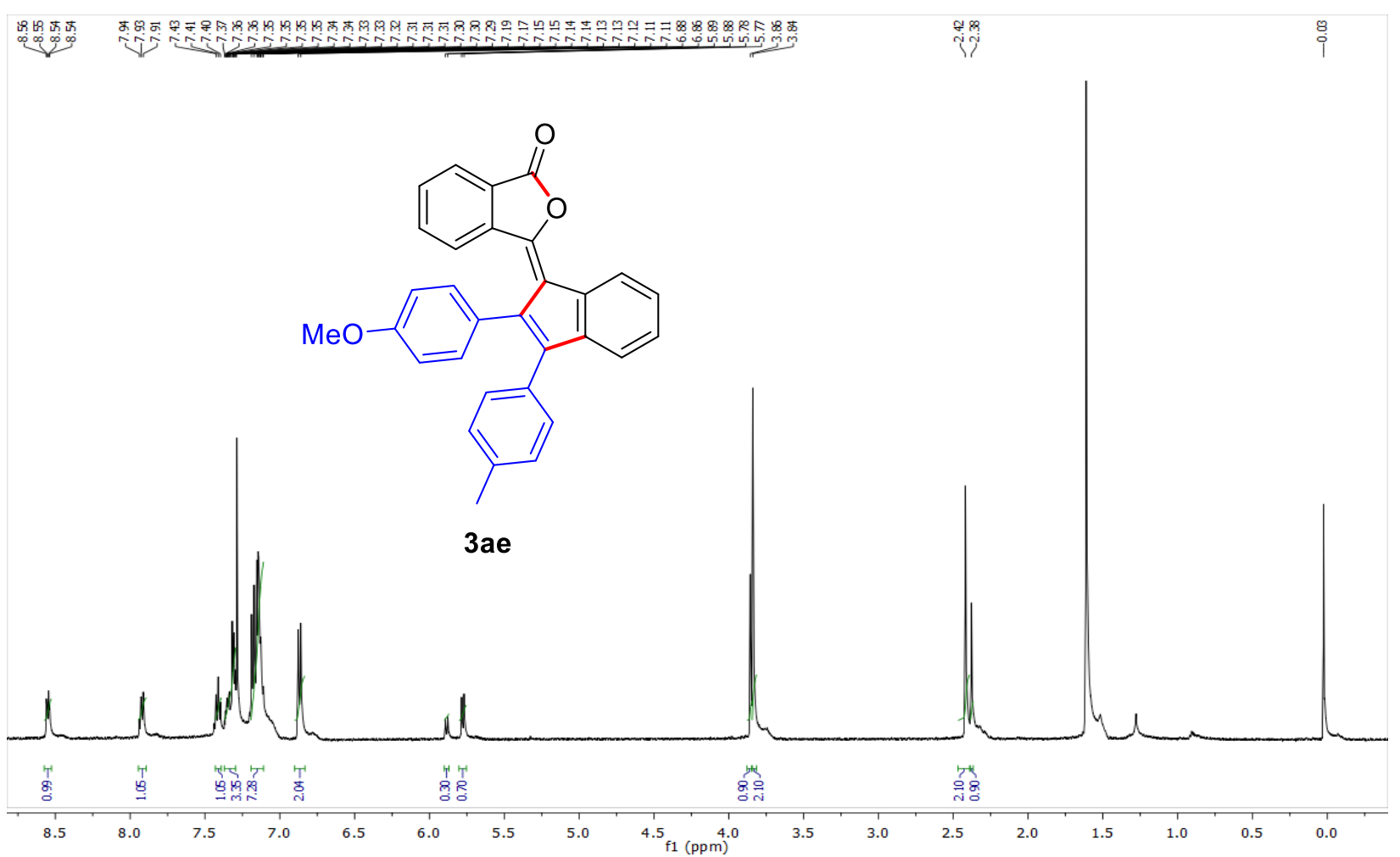

Expanded form of 3ae:

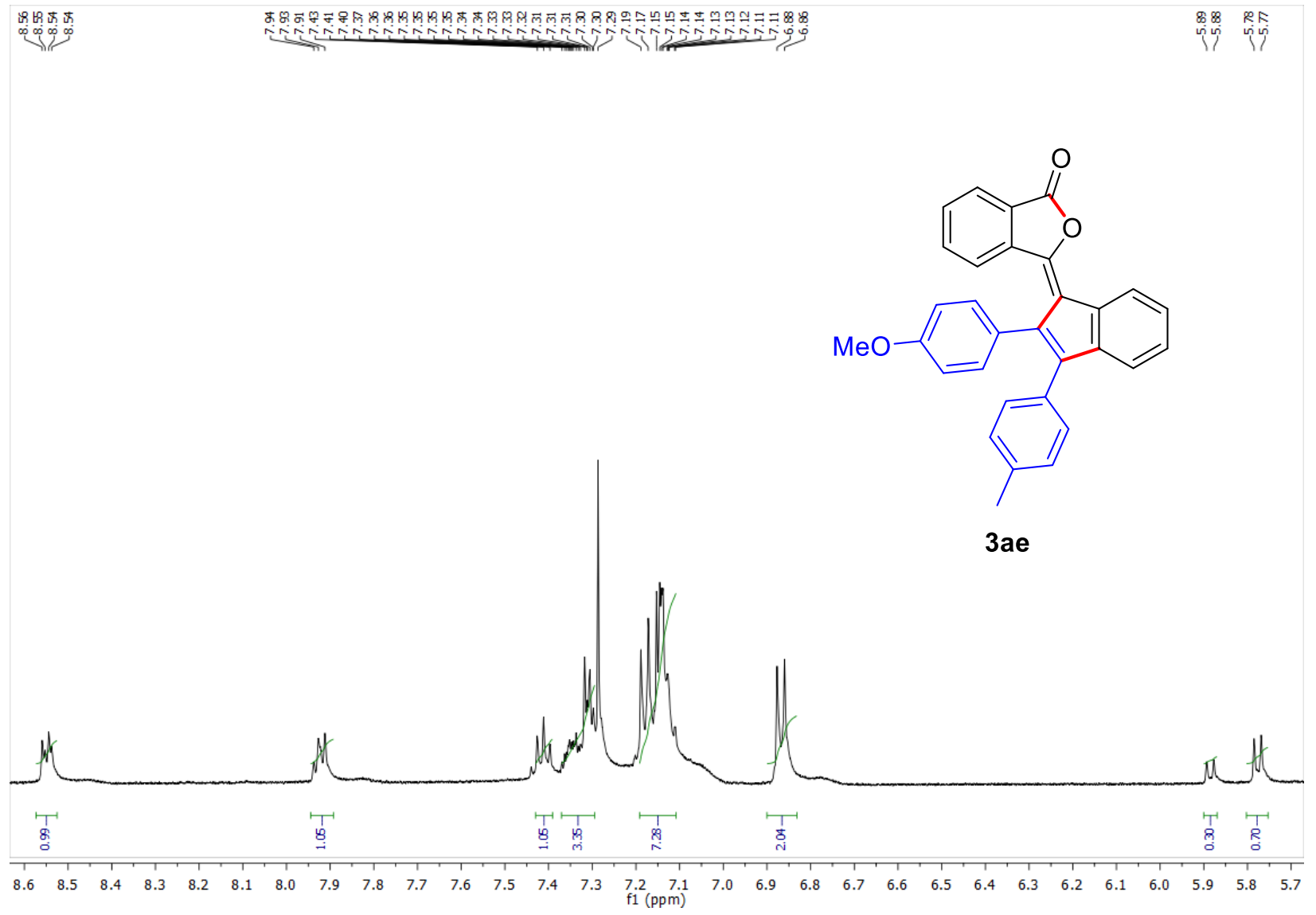



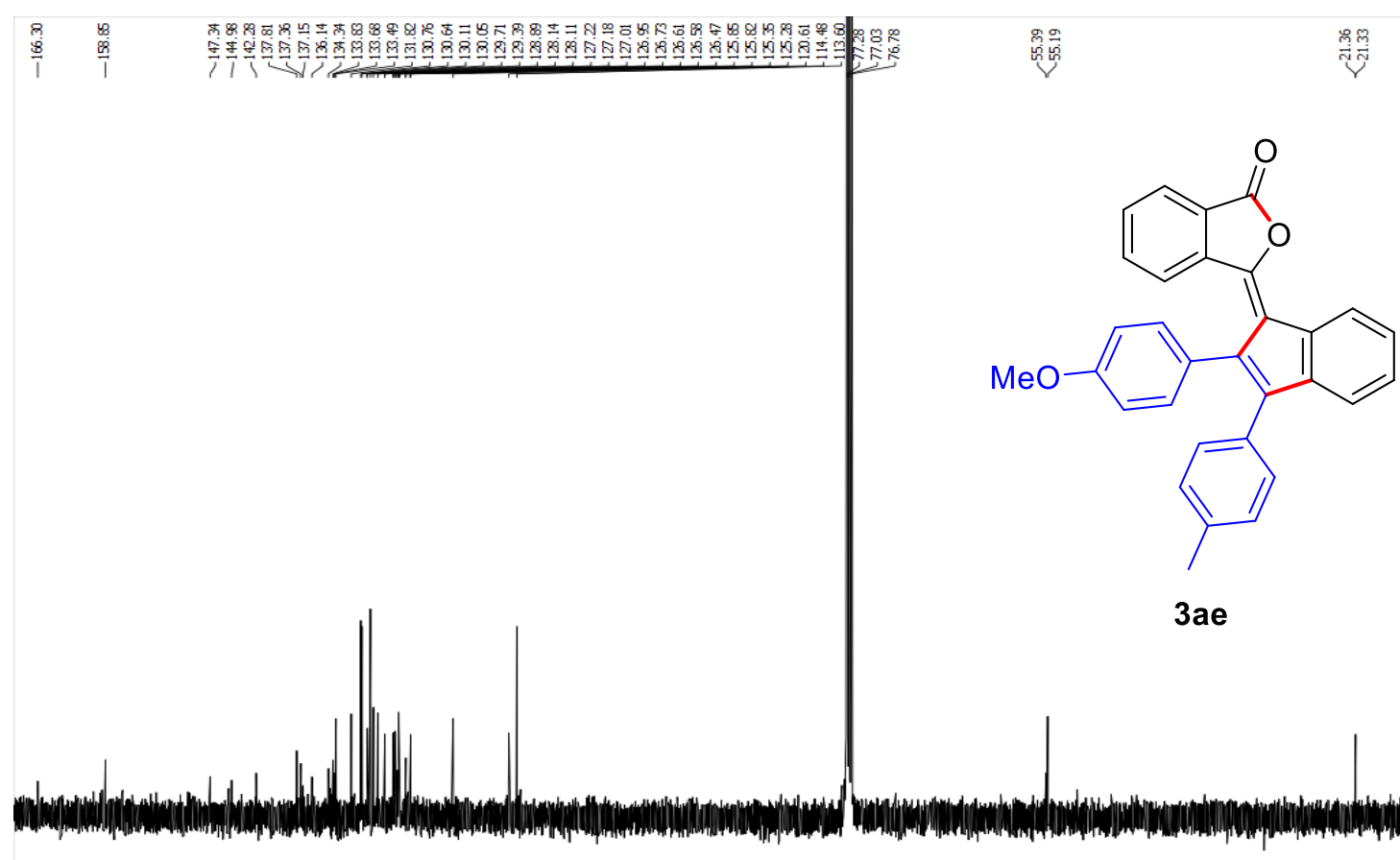

$3 a e$
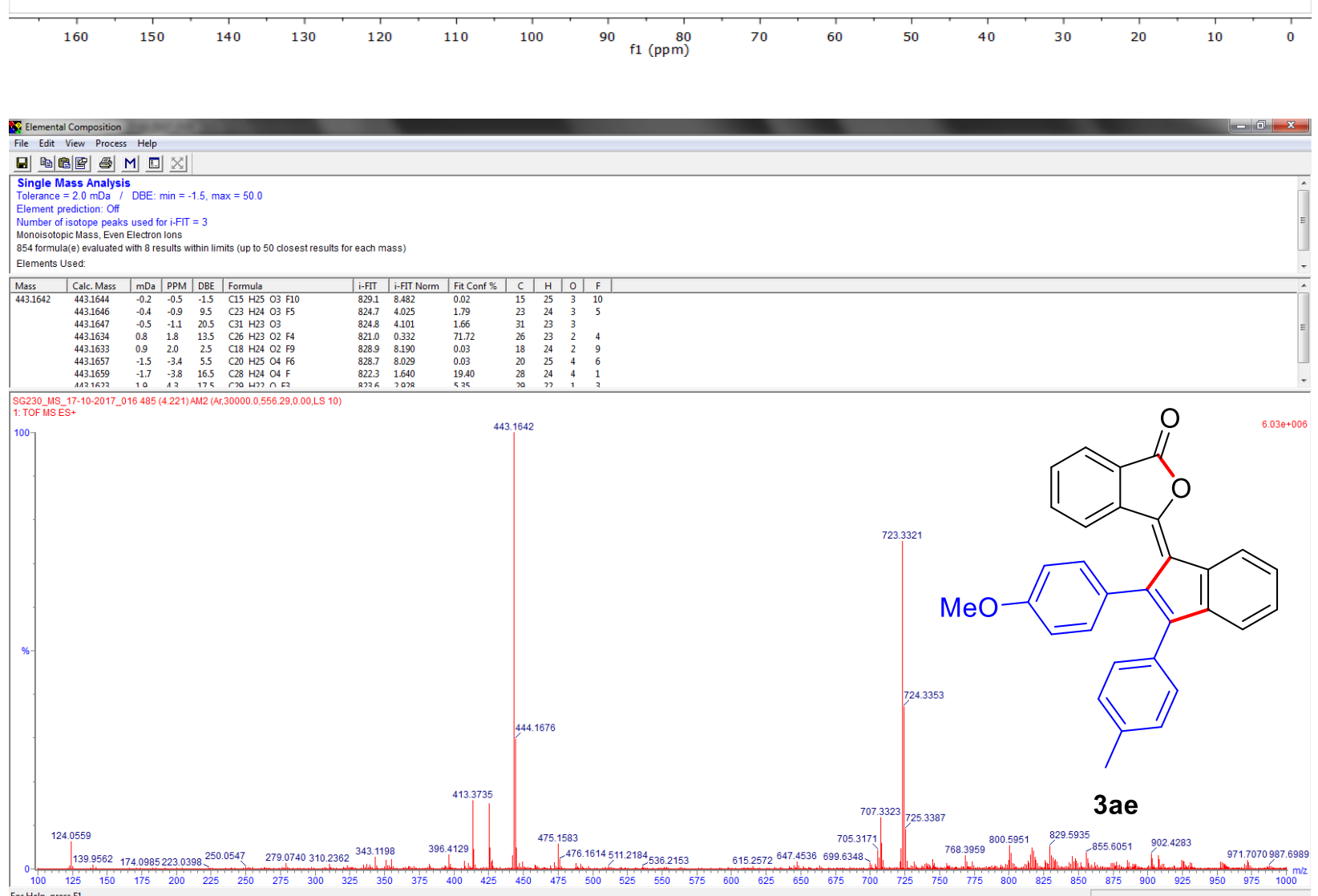


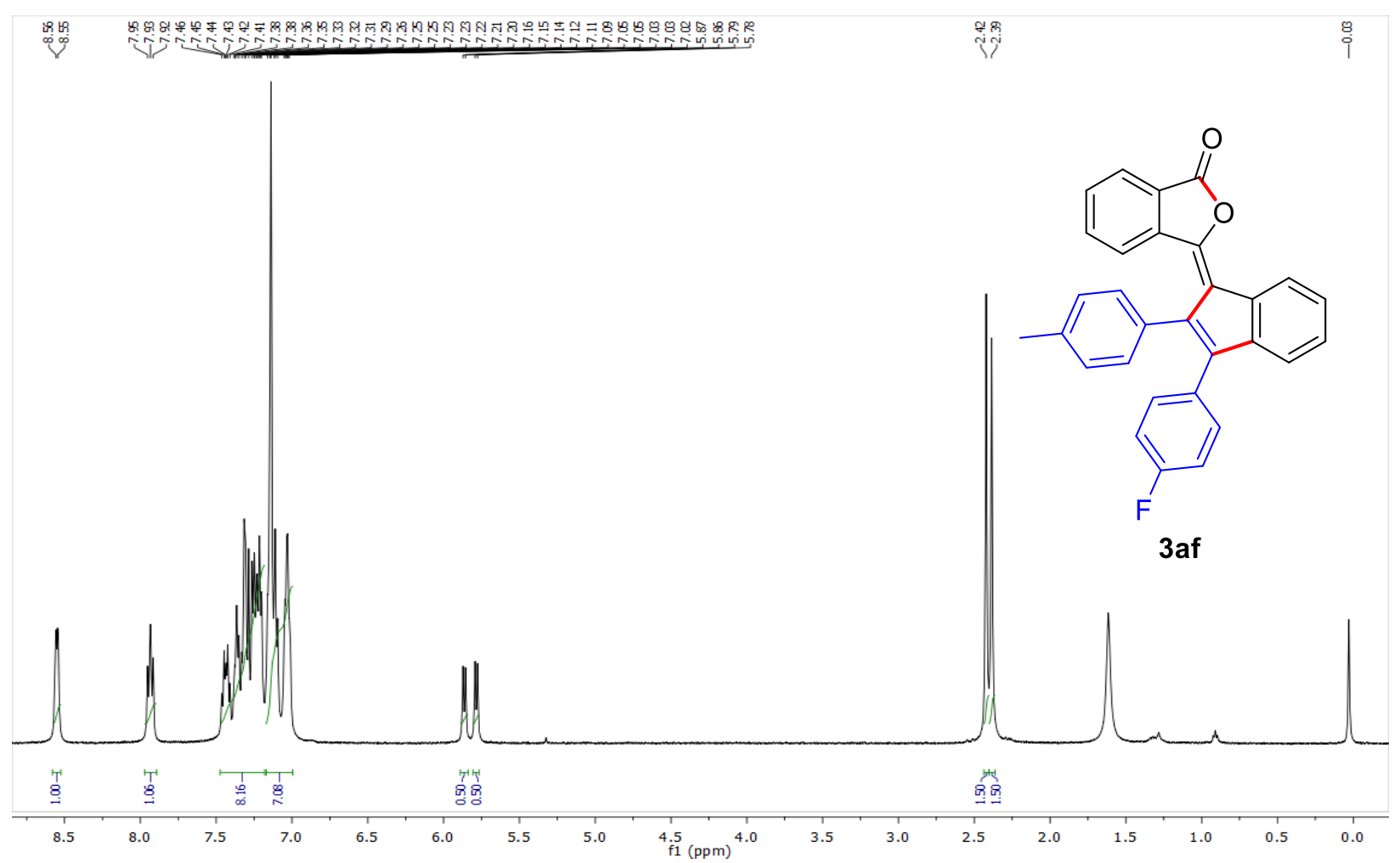

Expanded form of 3 af:

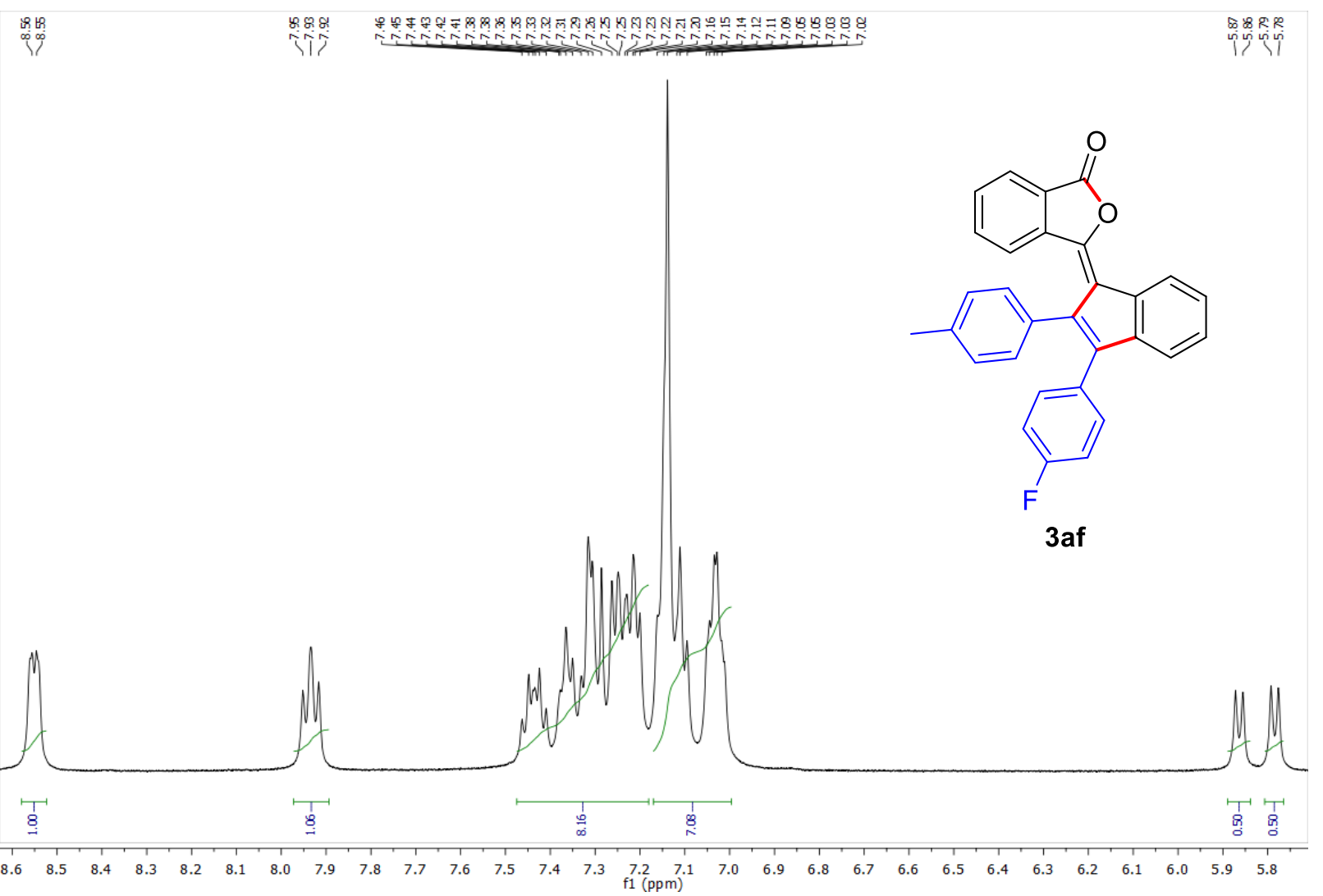



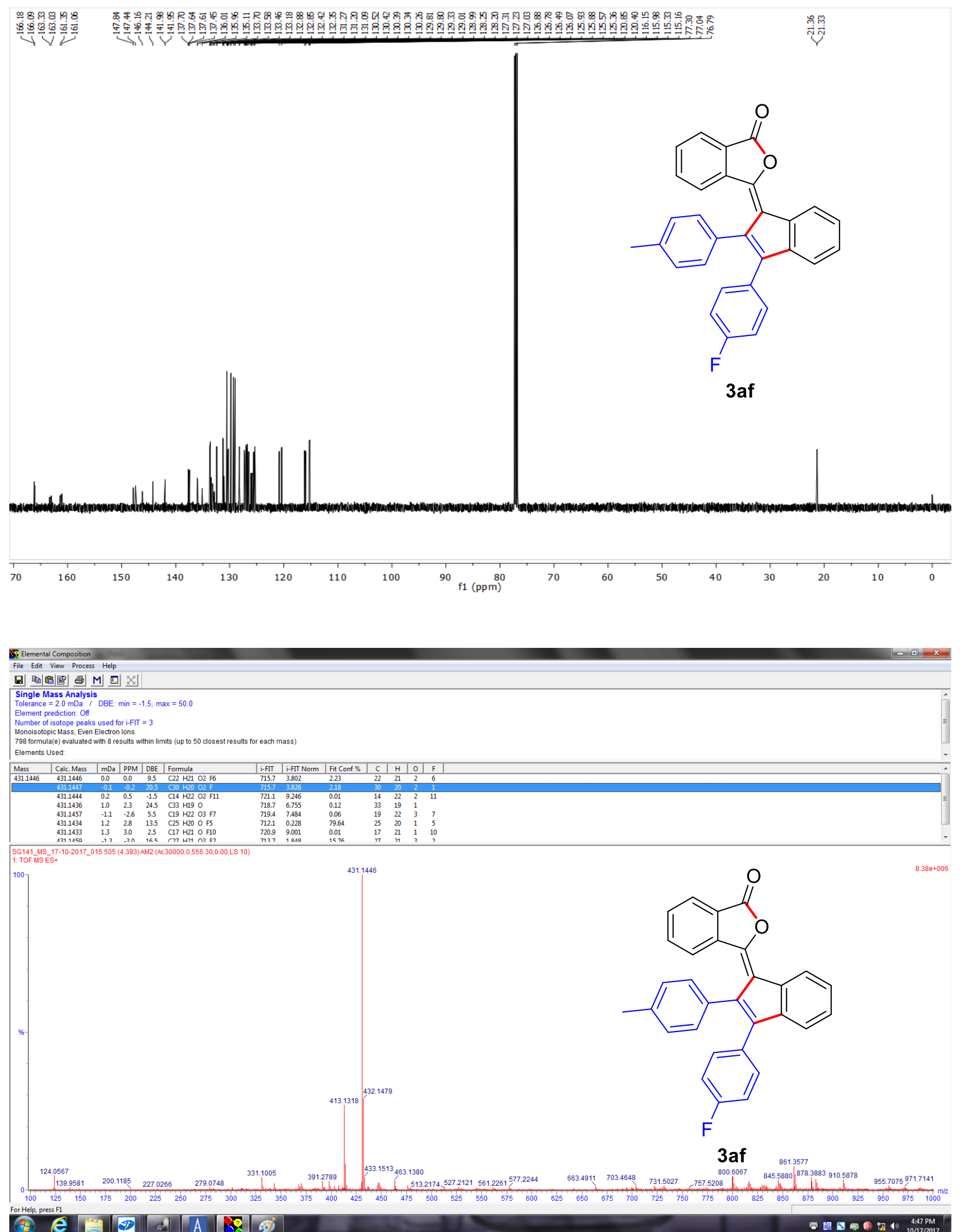


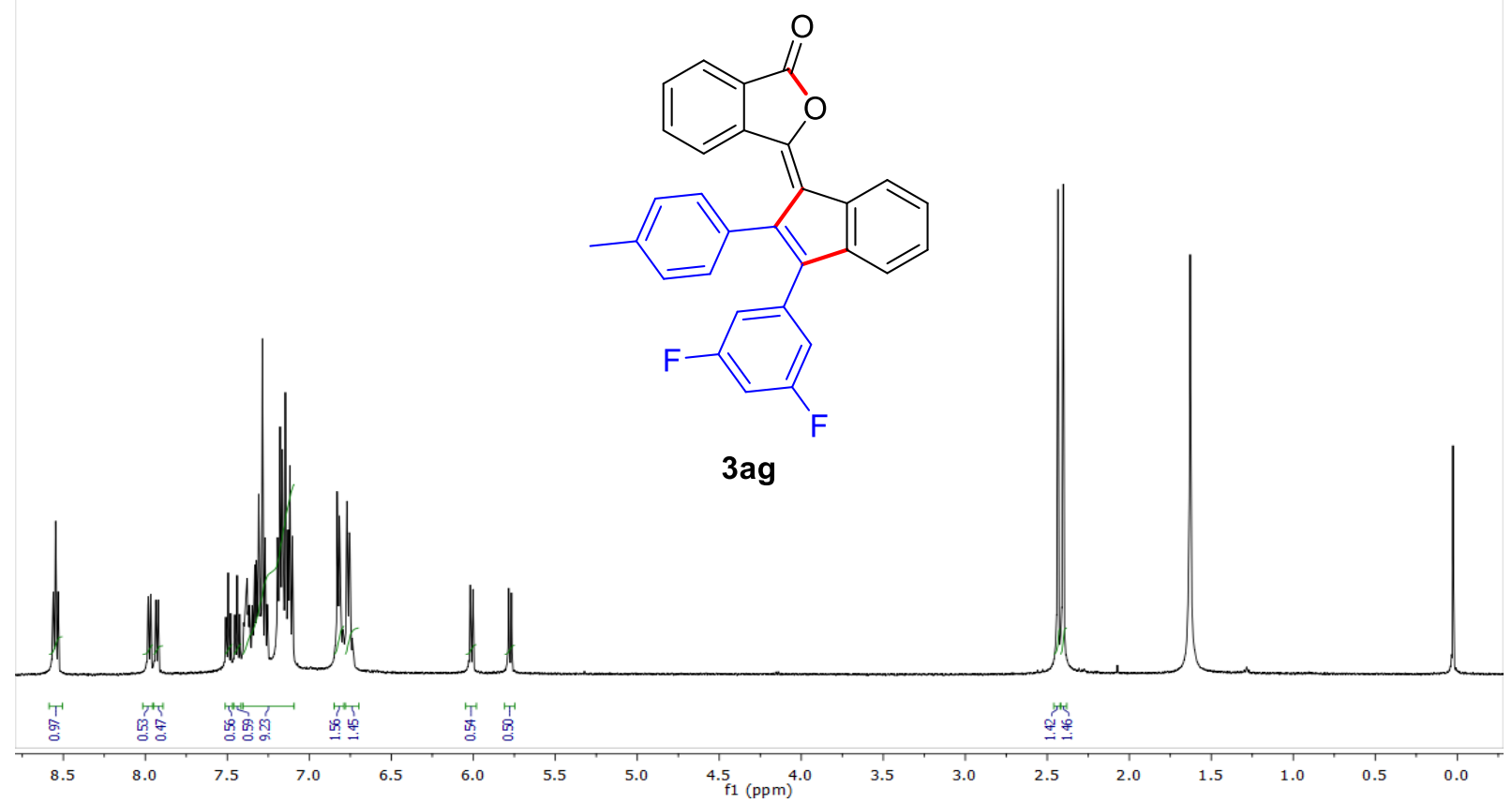

Expanded form of 3ag:

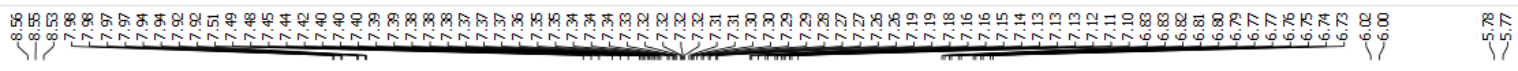

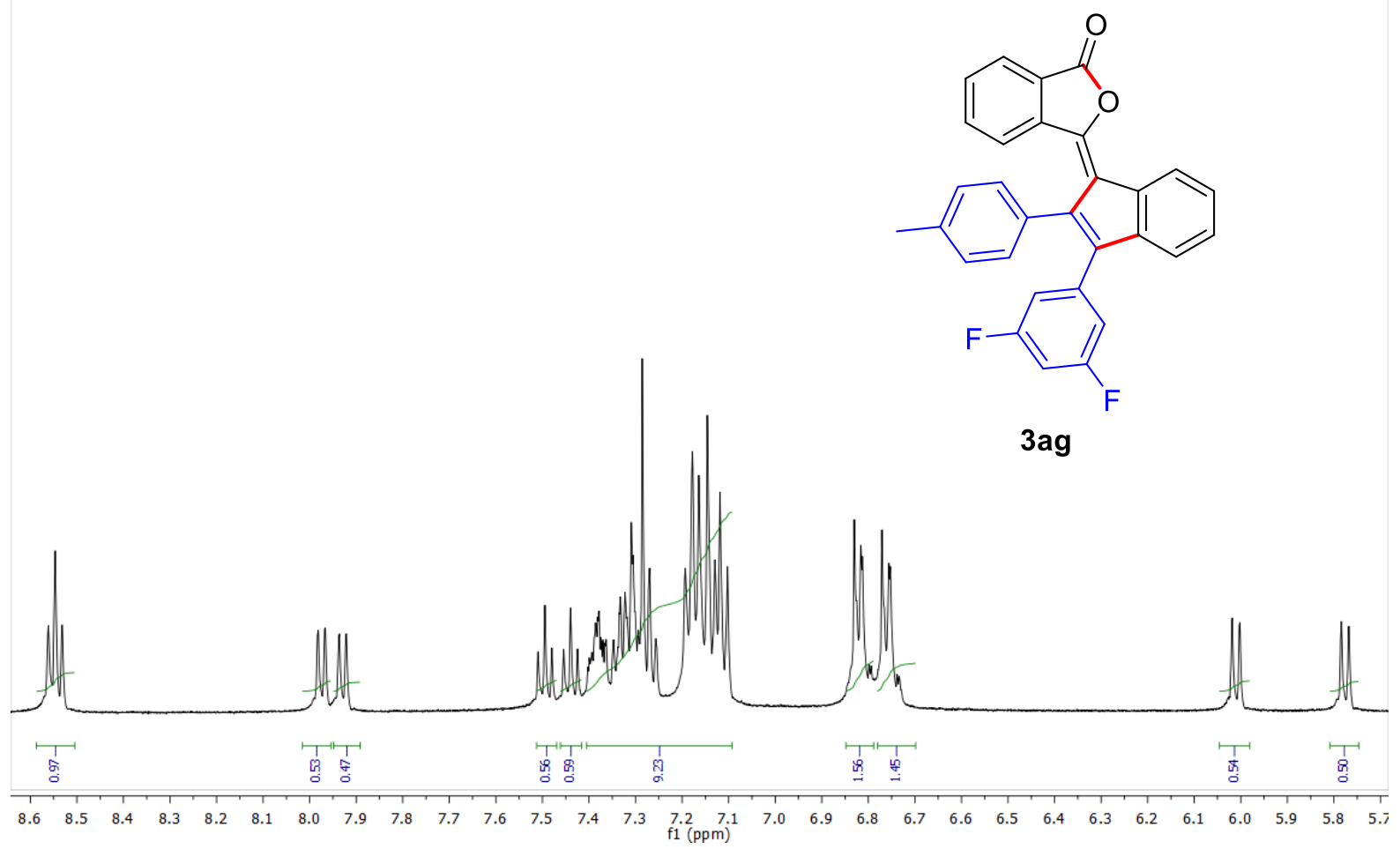



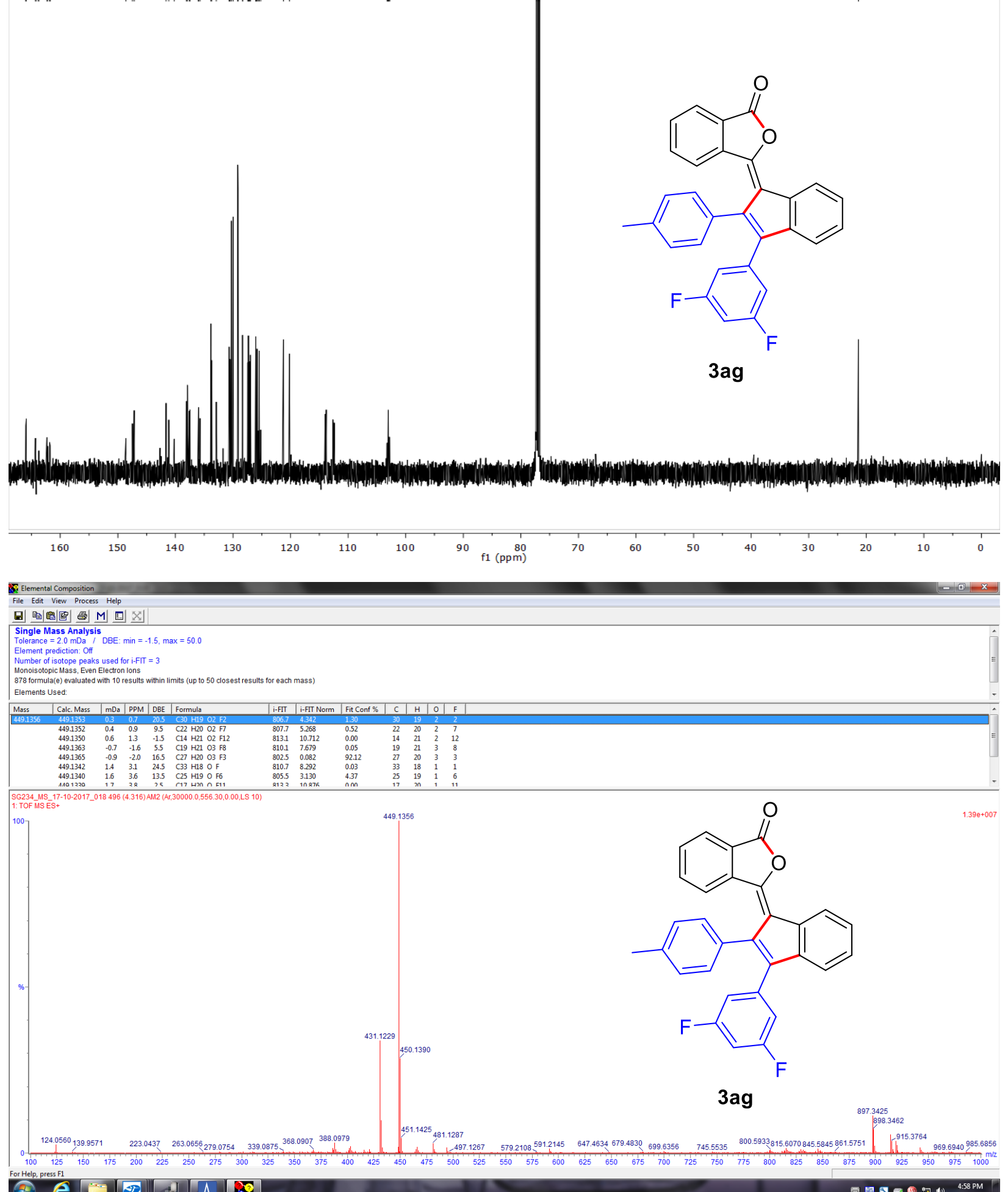


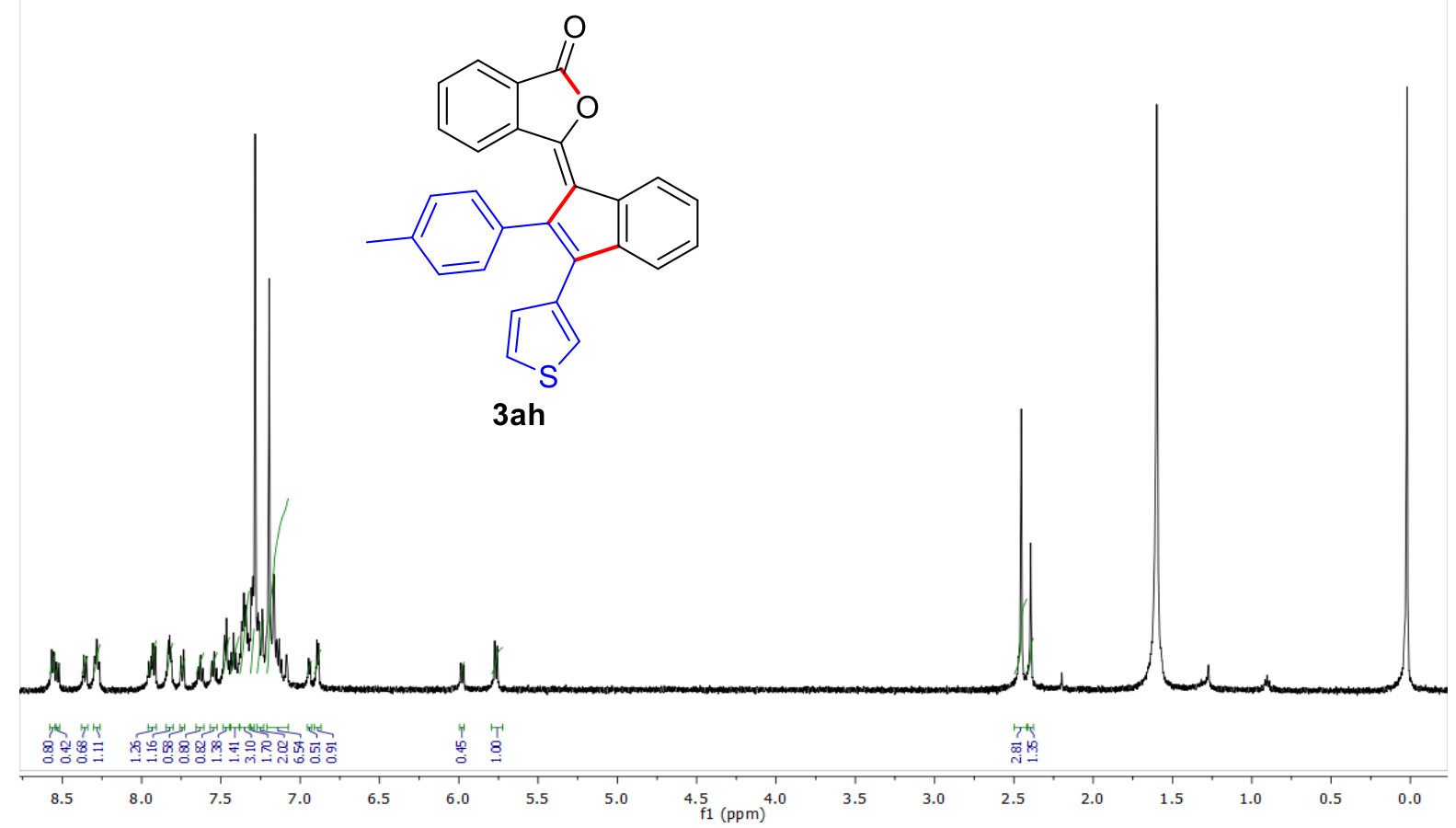

Expanded form of 3ah:

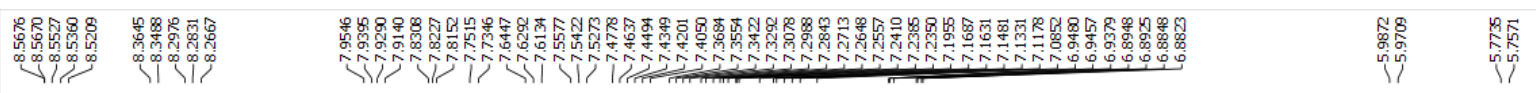

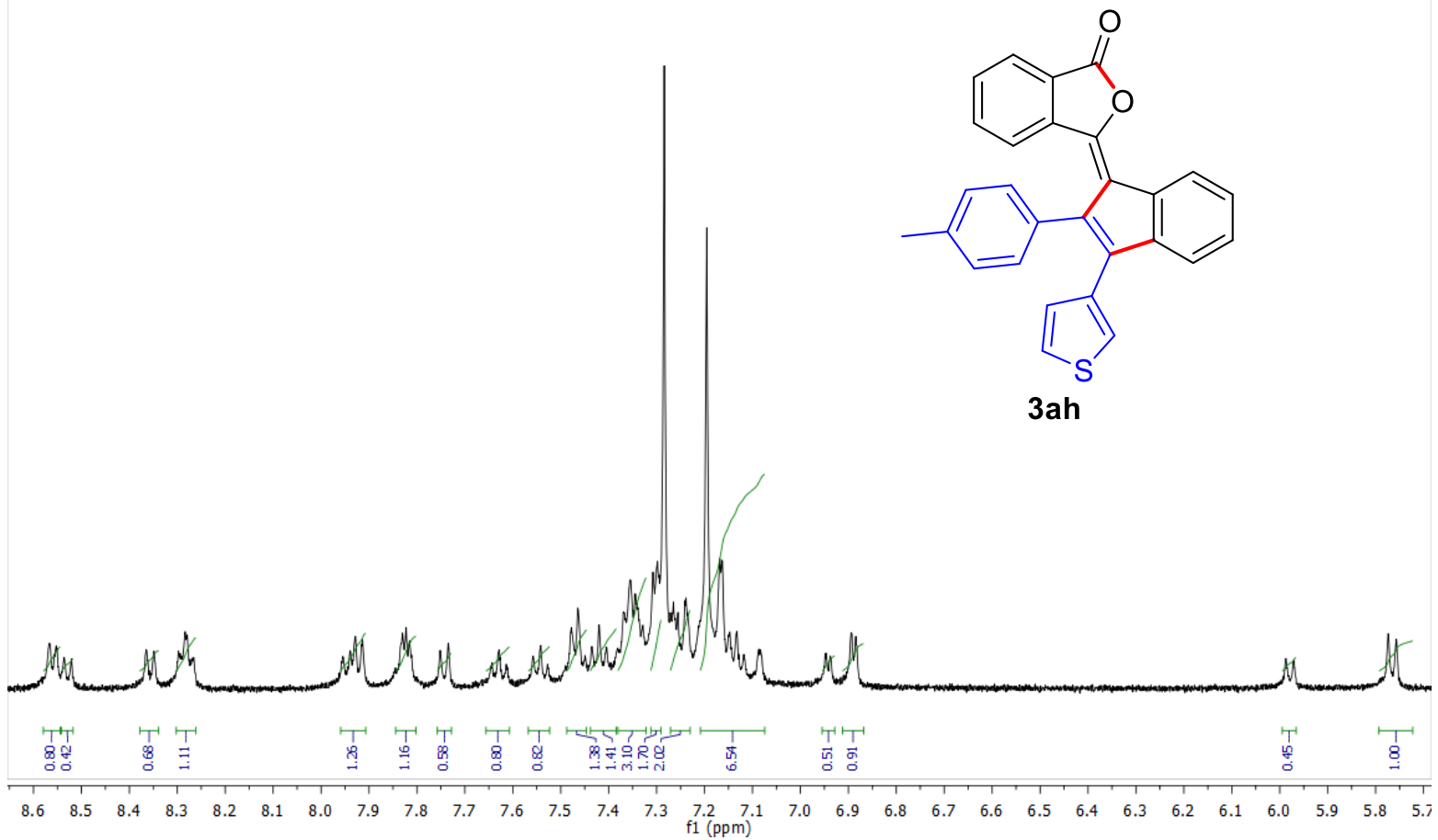



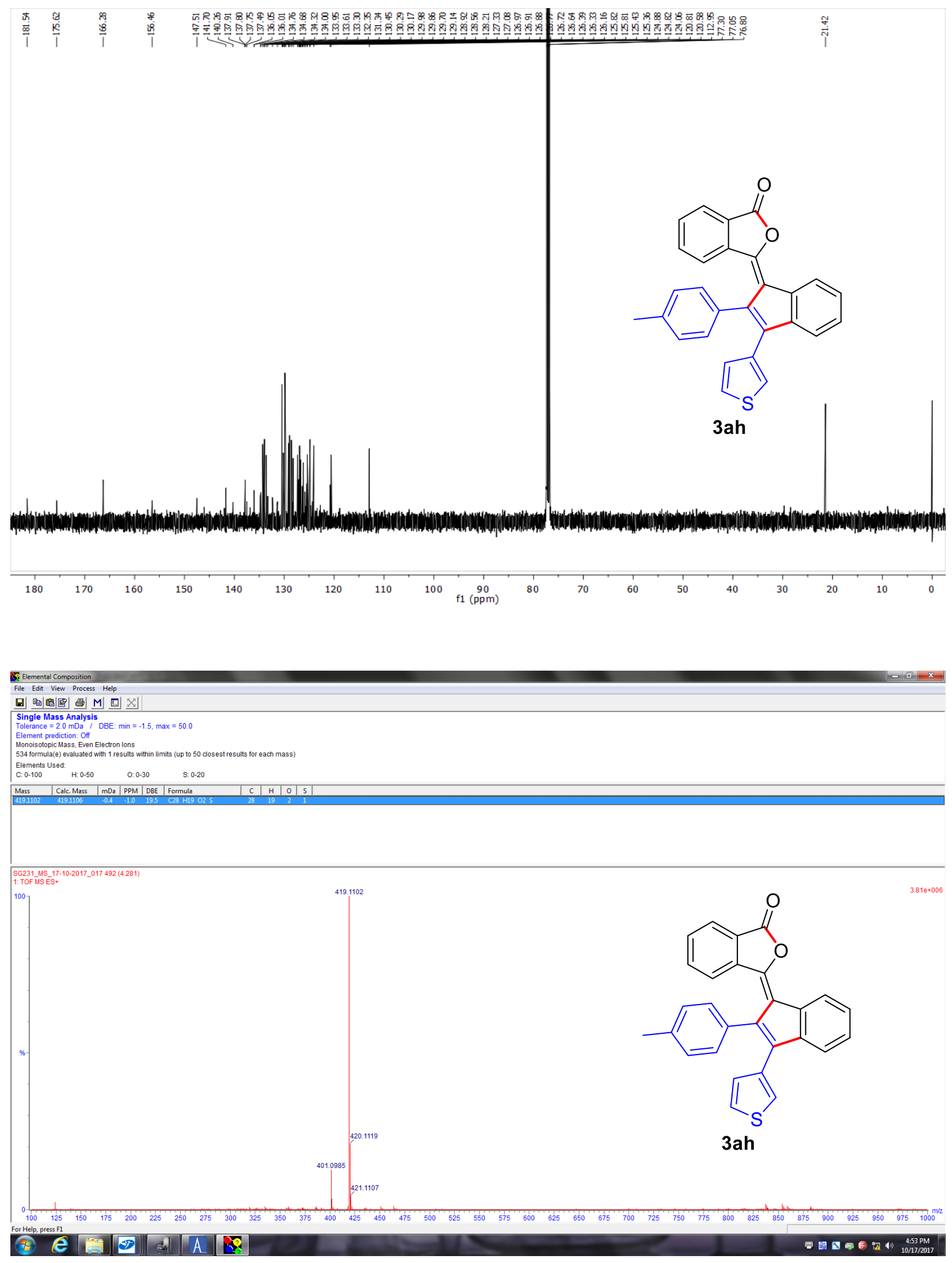


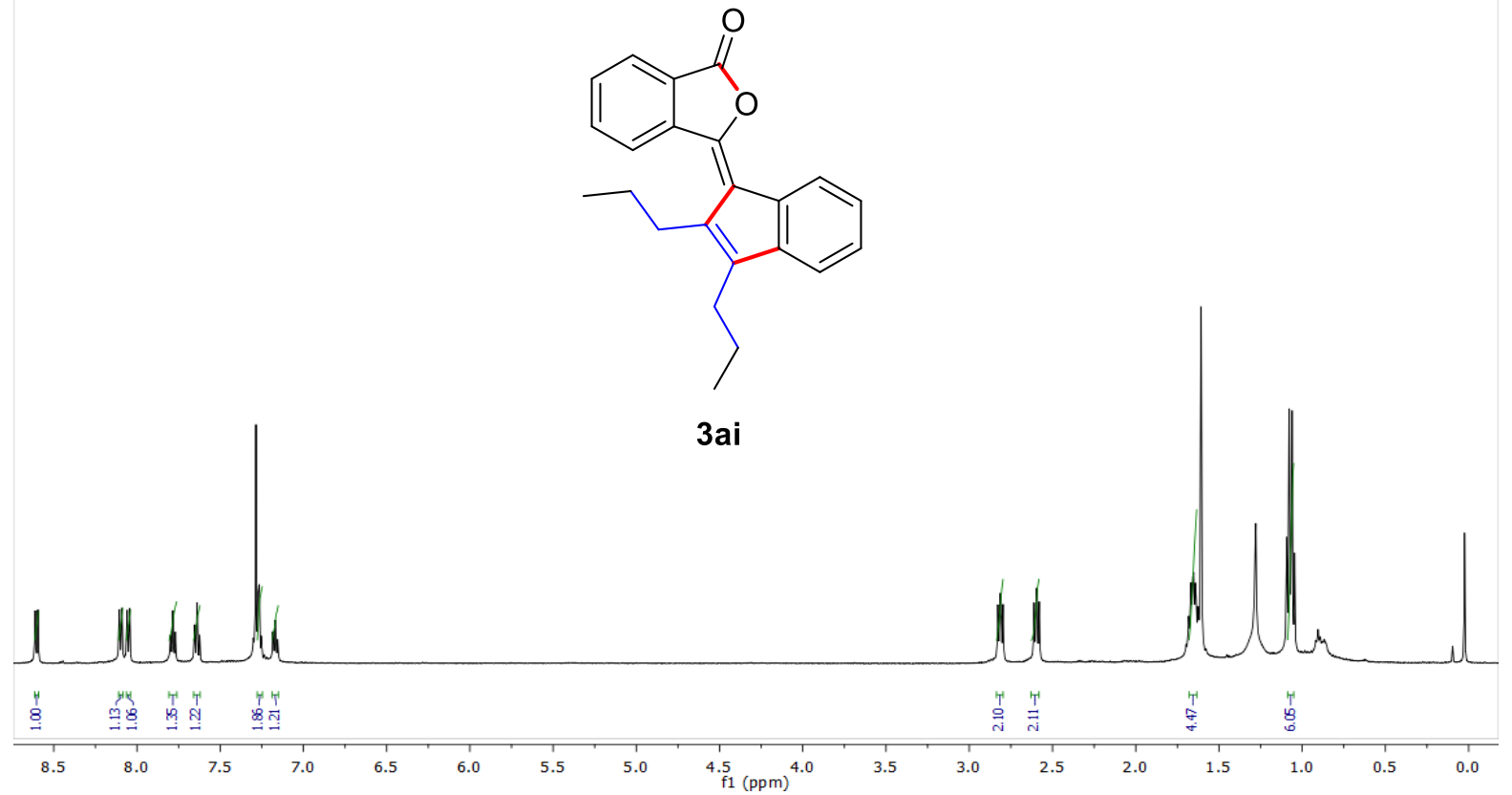

Expanded form of 3ai:

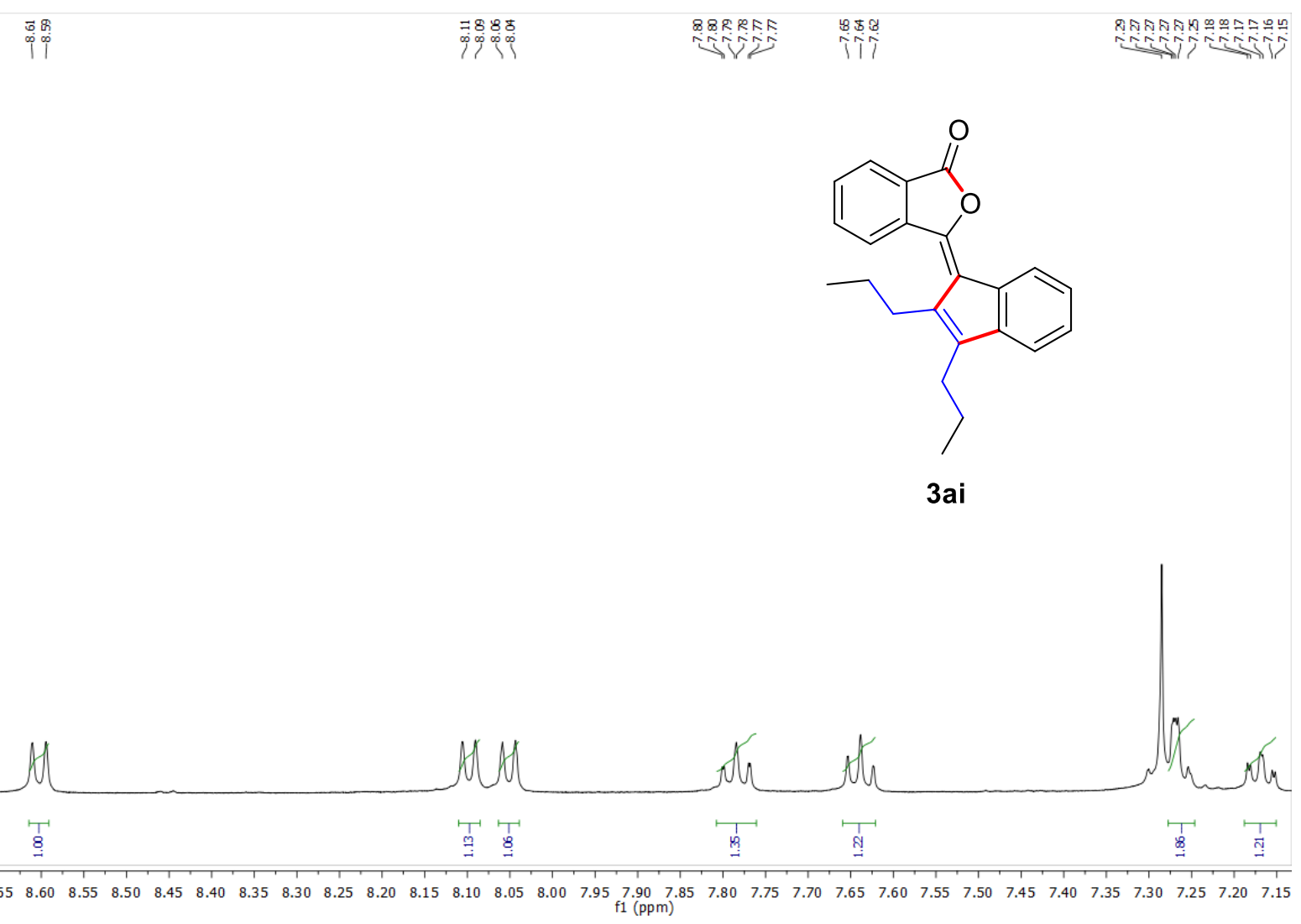



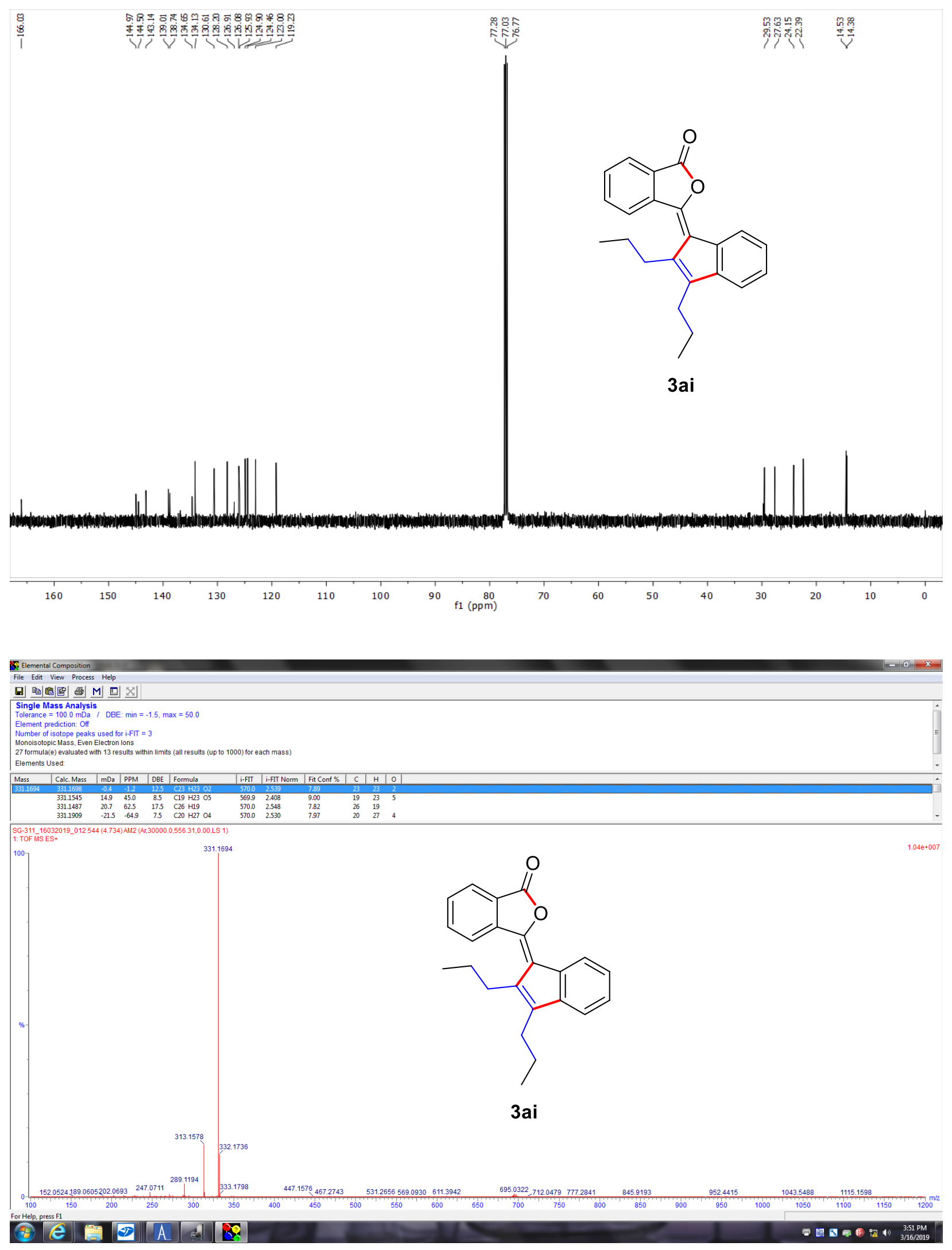


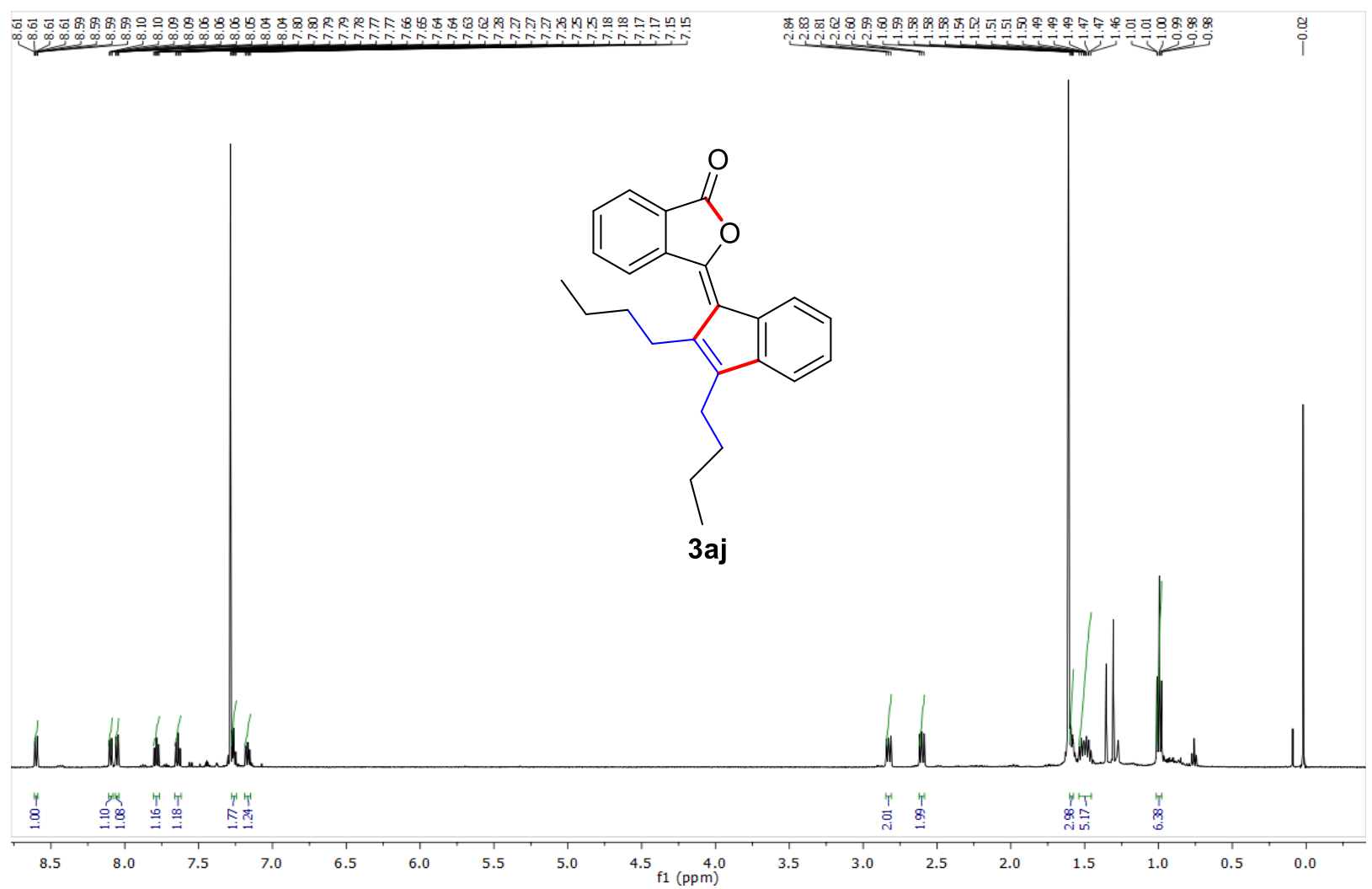

Expanded form of 3aj:

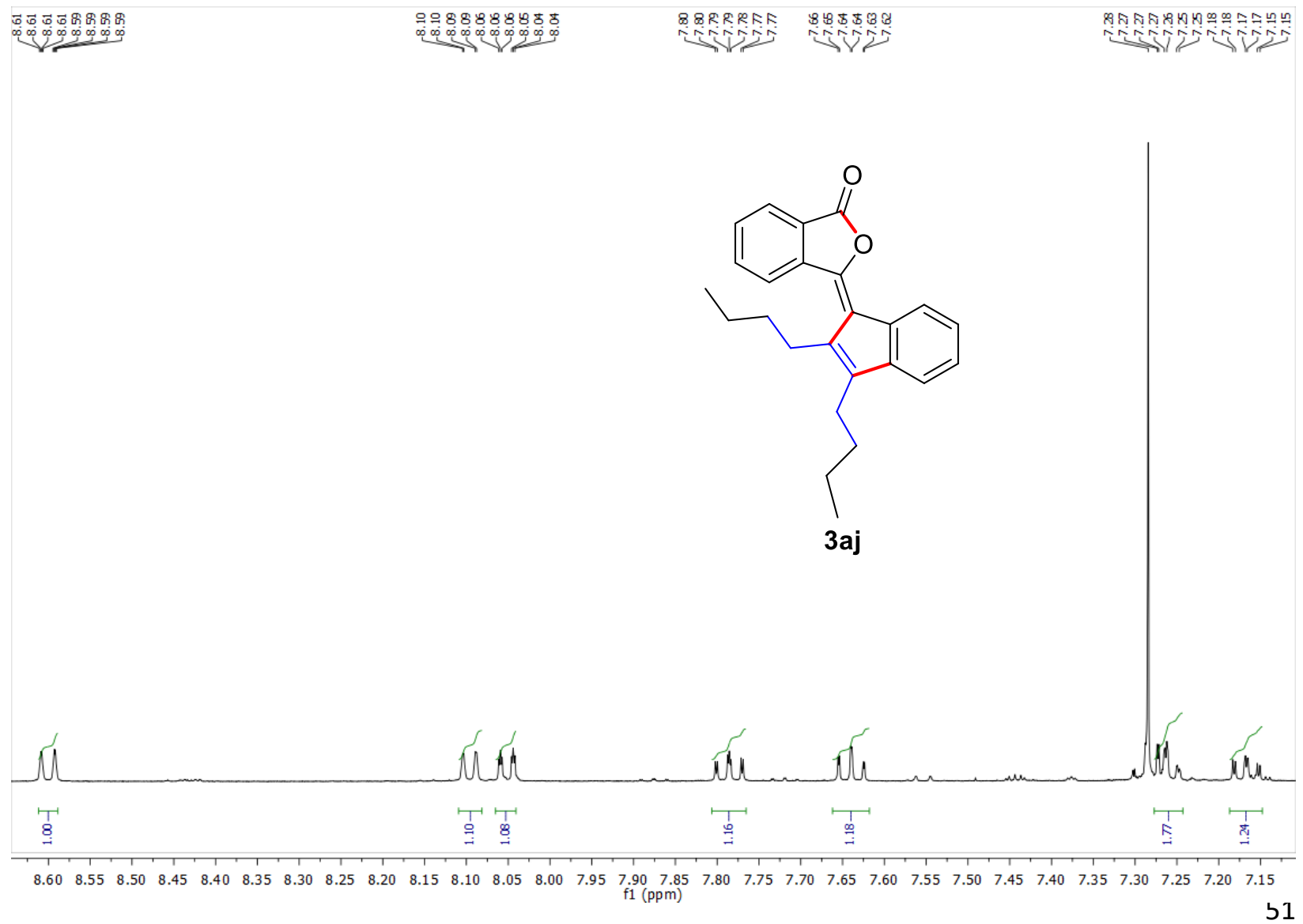



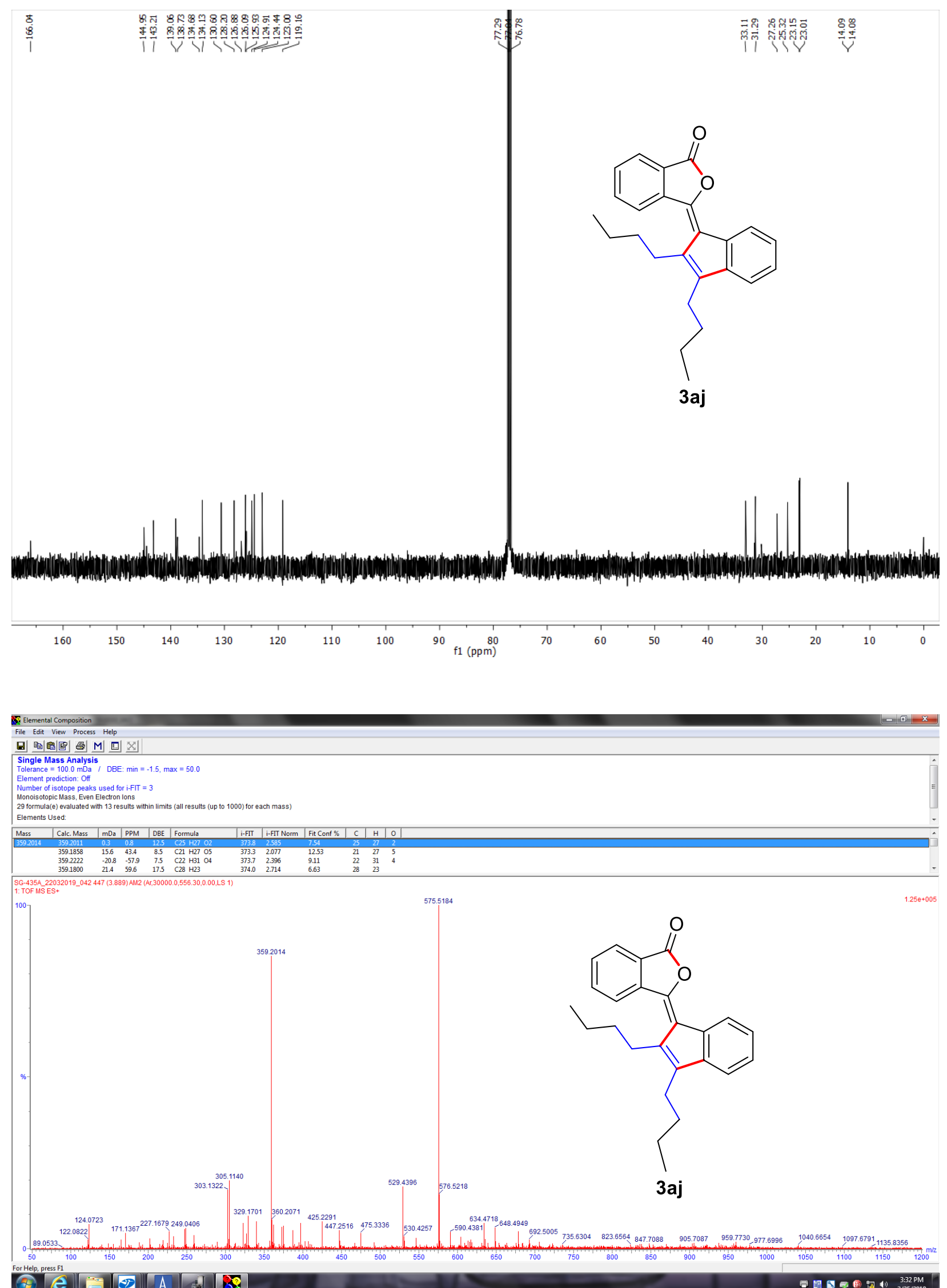

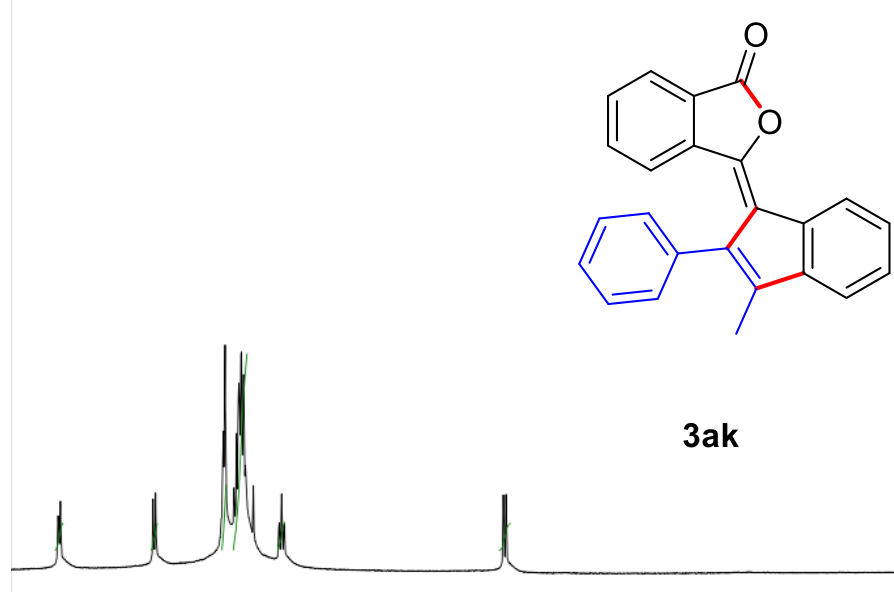

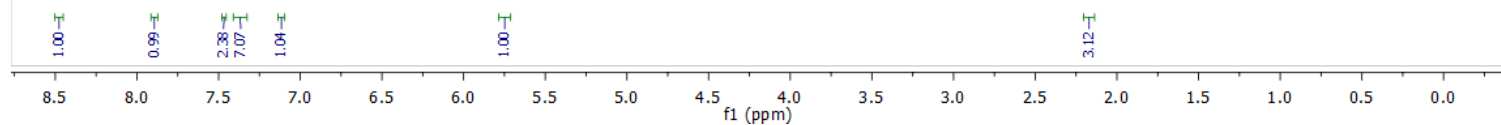

Expanded form of 3ak:
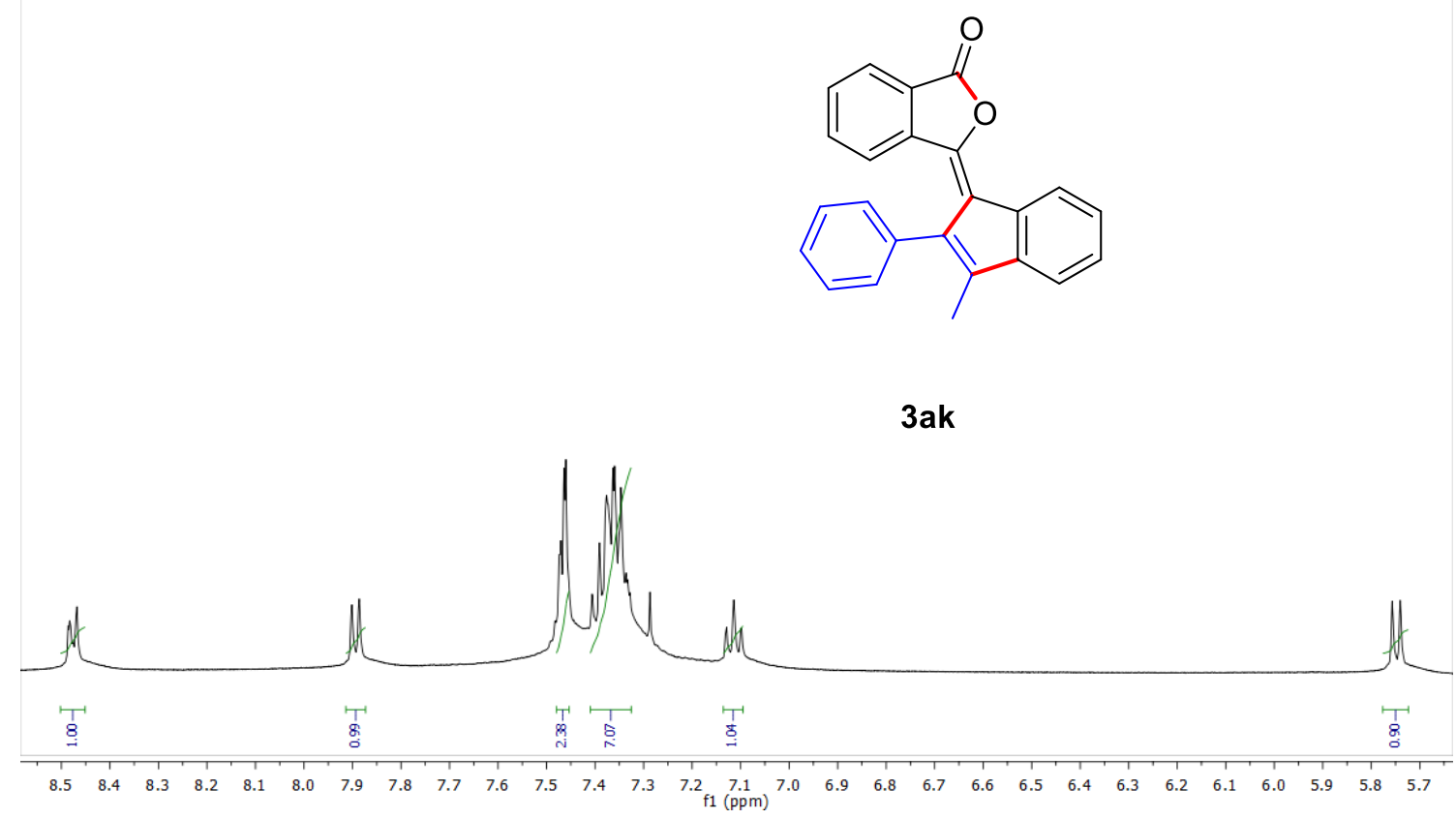
<smiles>CC1=C(c2ccccc2)/C(=C2\OC(=O)c3ccccc32)c2ccccc21</smiles>

3ak

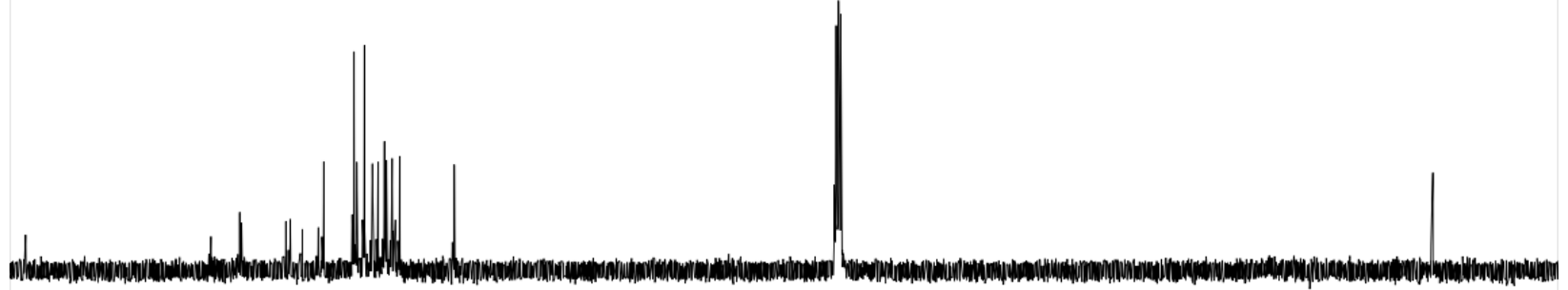

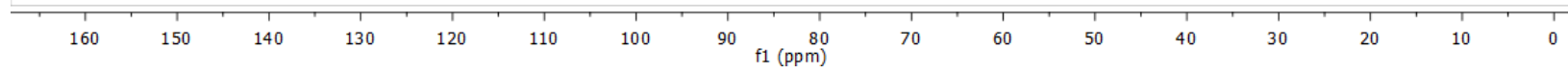

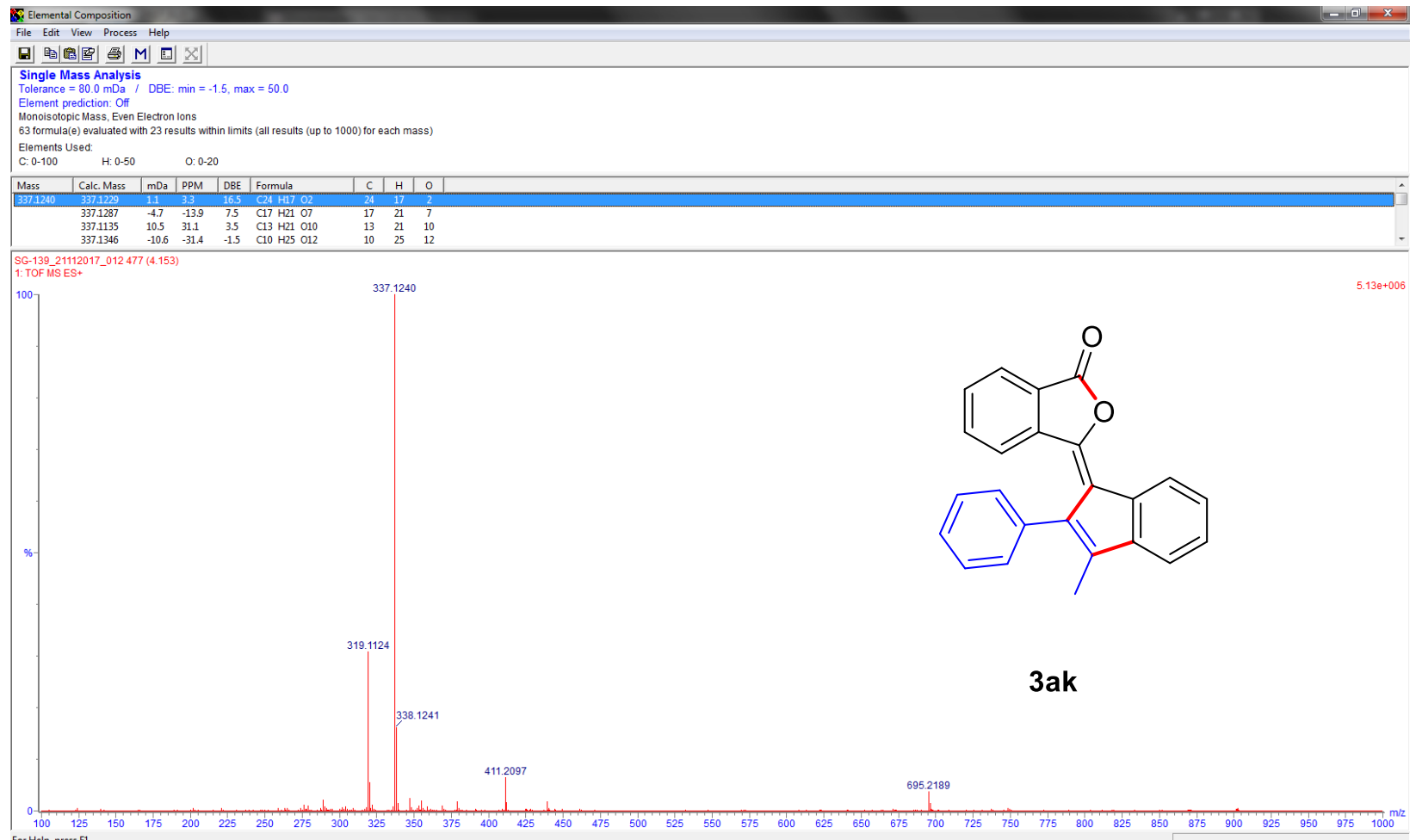




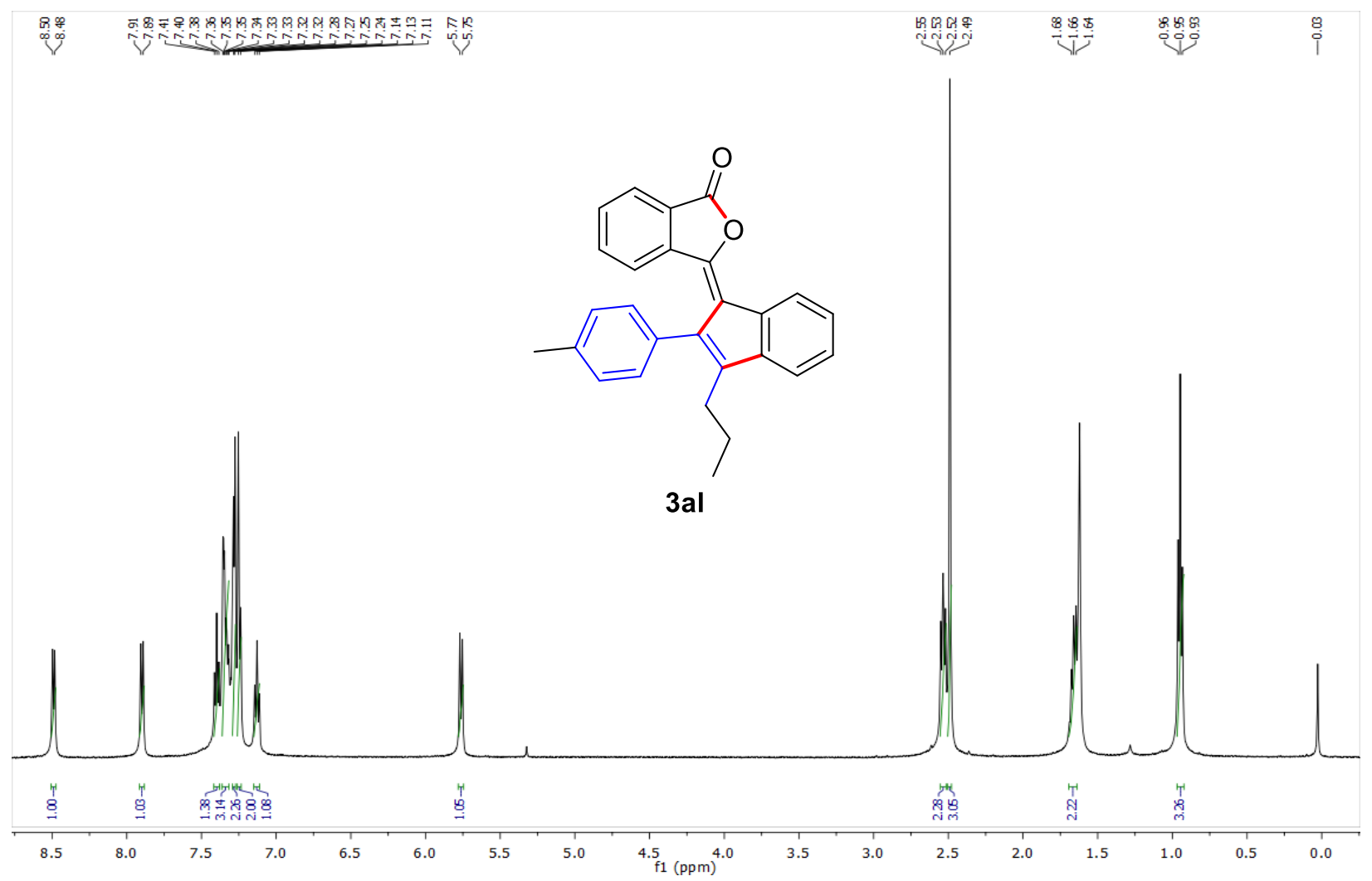

Expanded form of 3al:

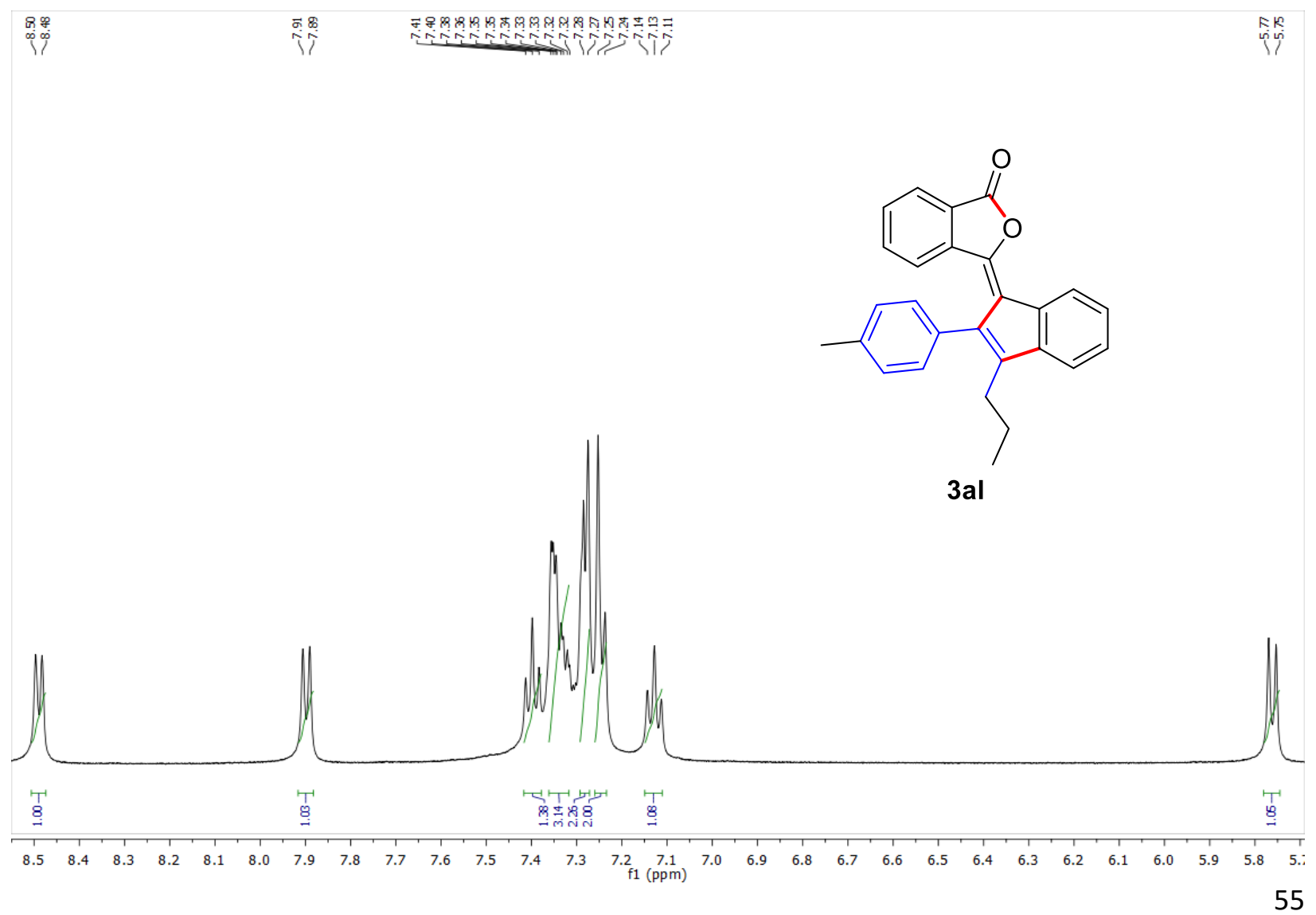



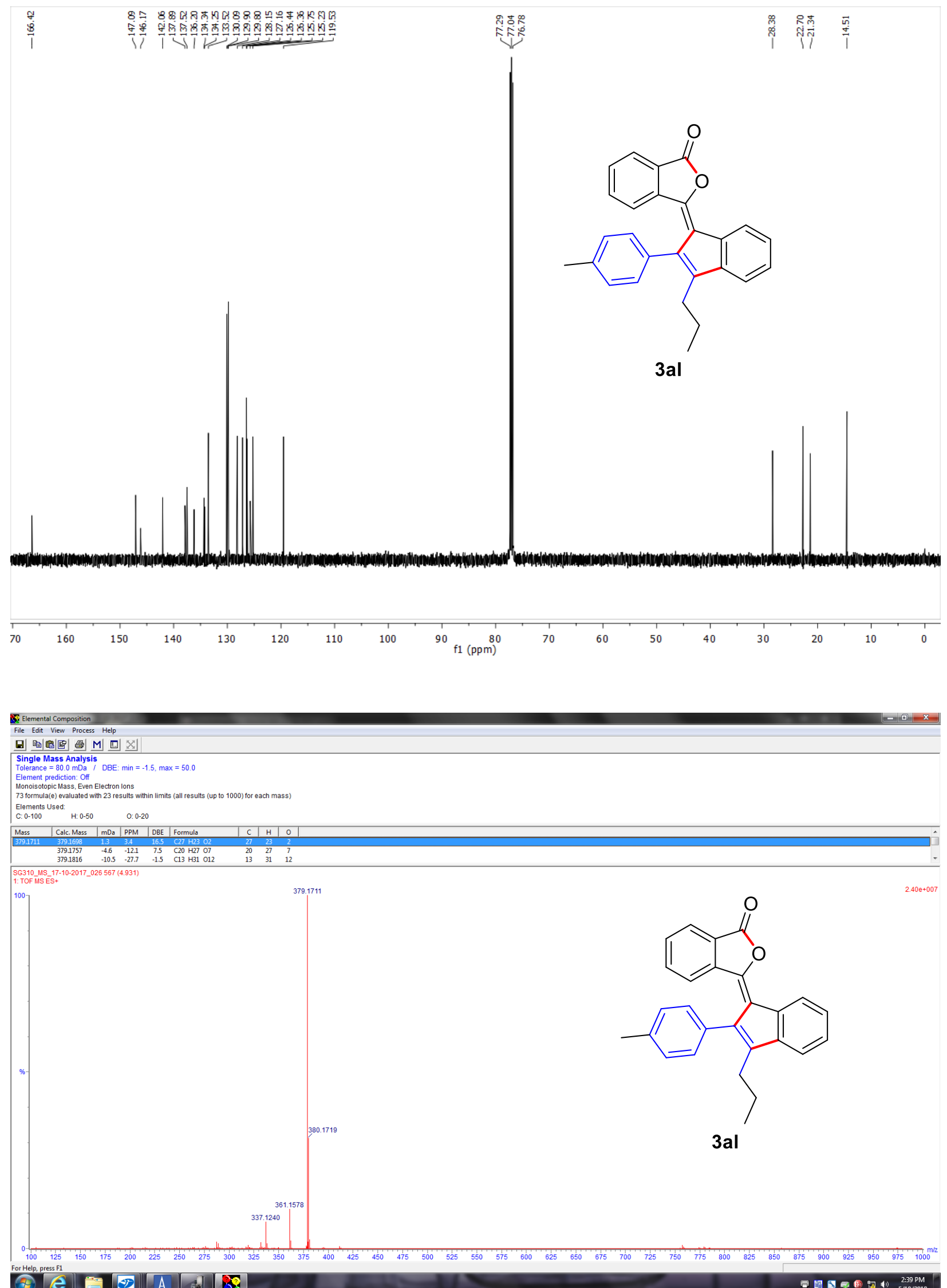


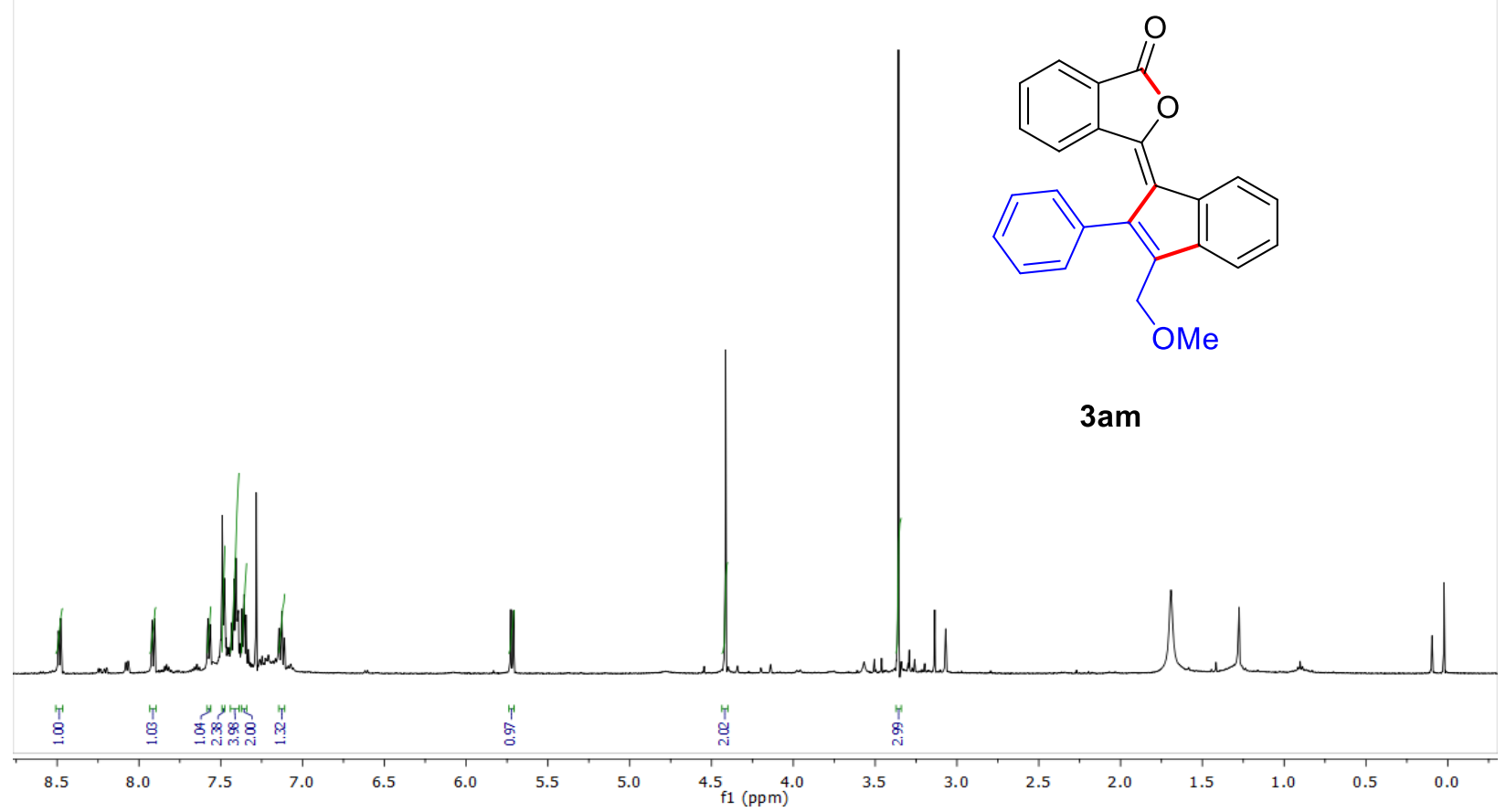

Expanded form of 3am:

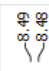

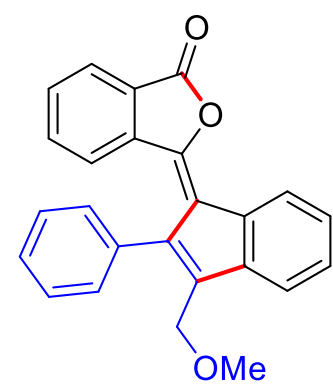

$3 a m$

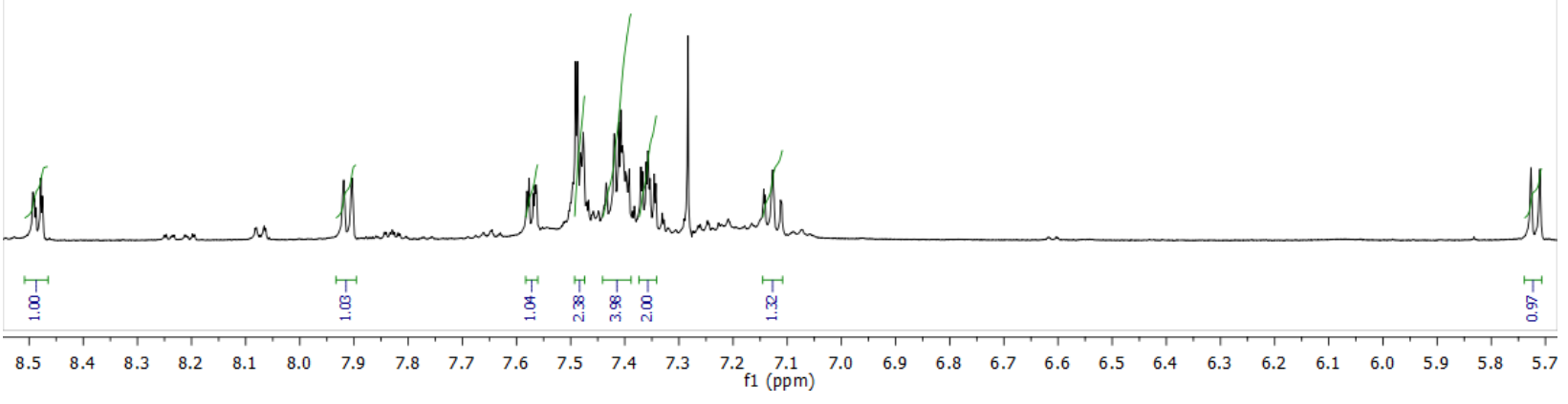




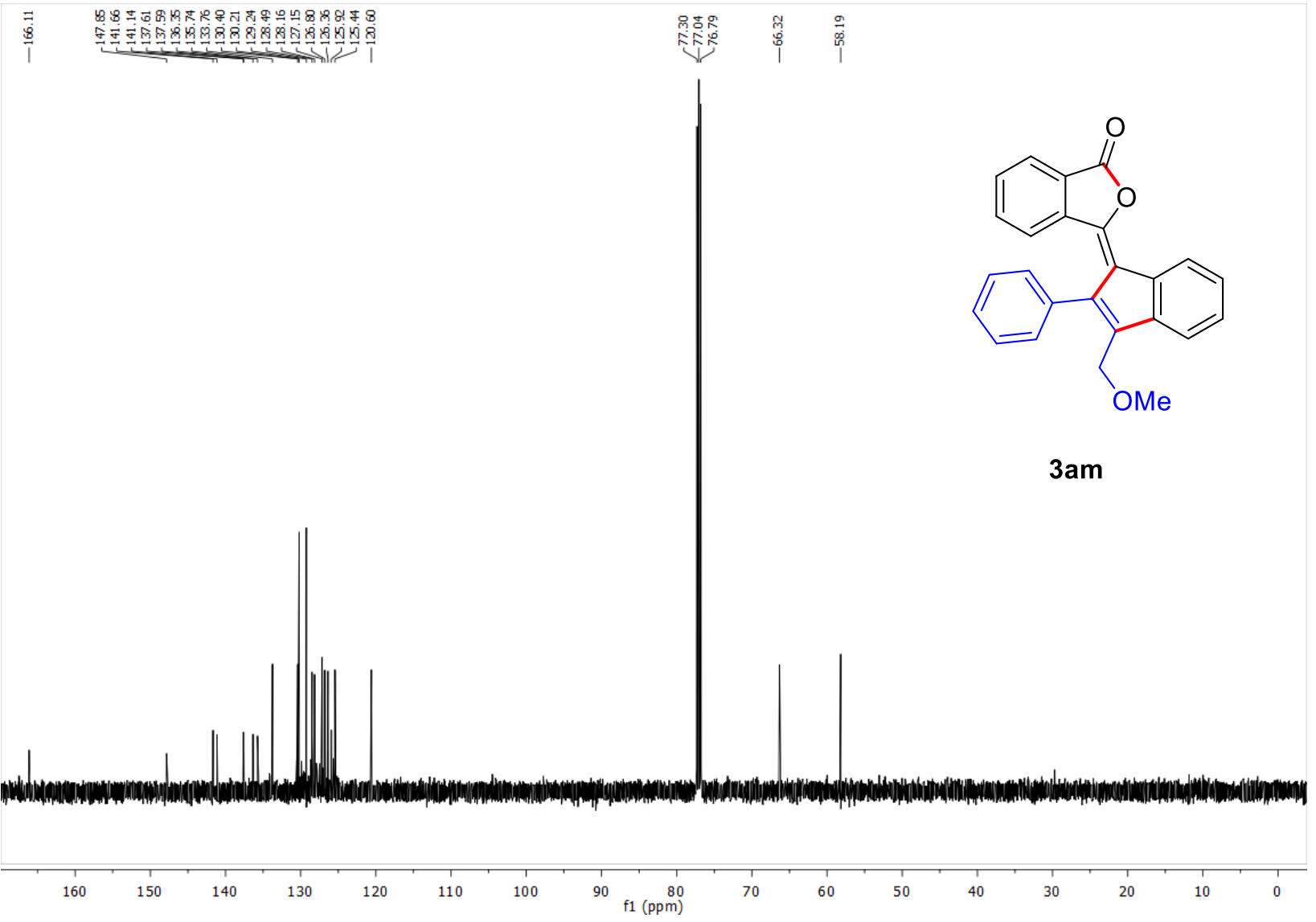




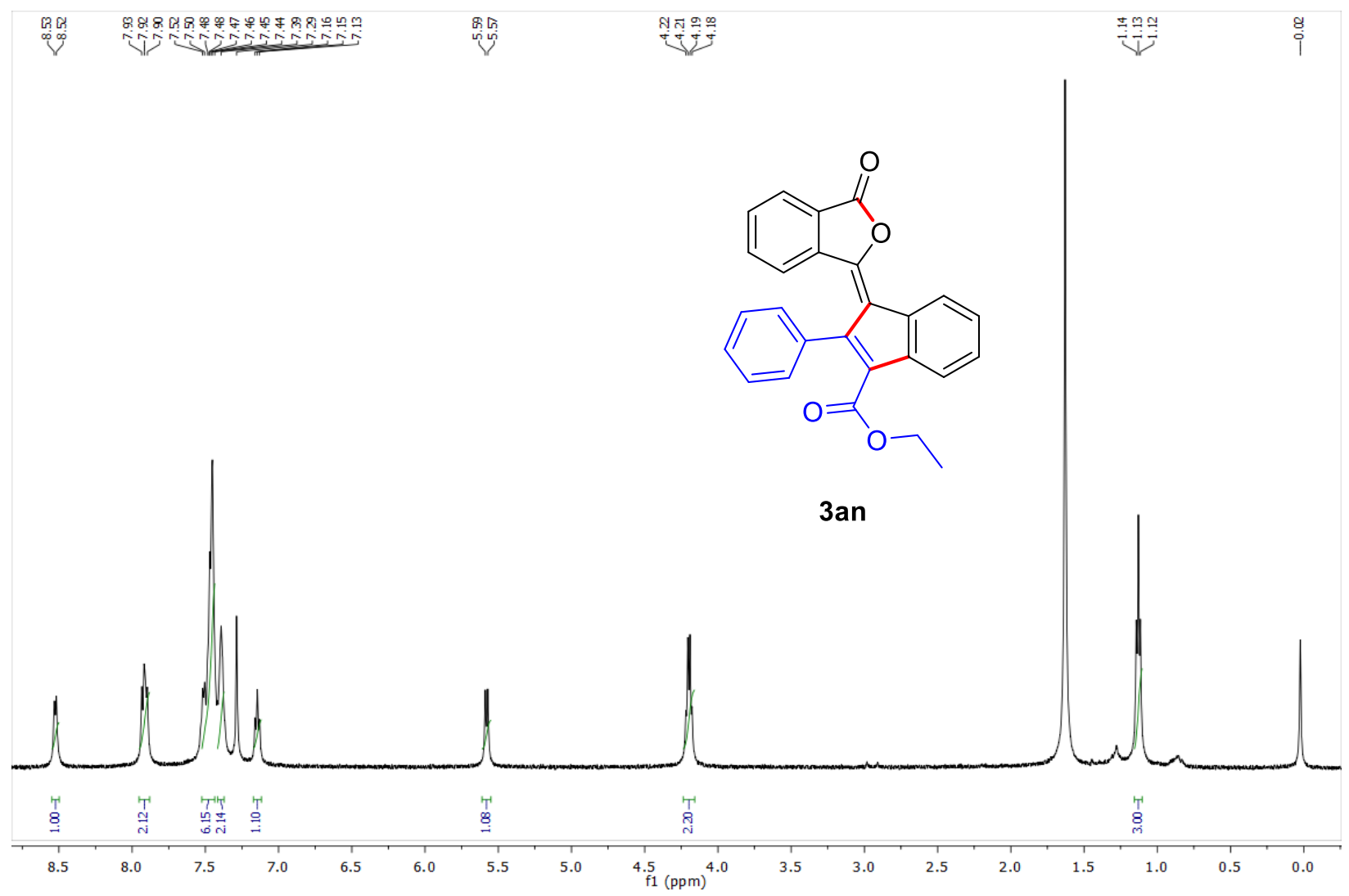

Expanded form of 3an:

娄

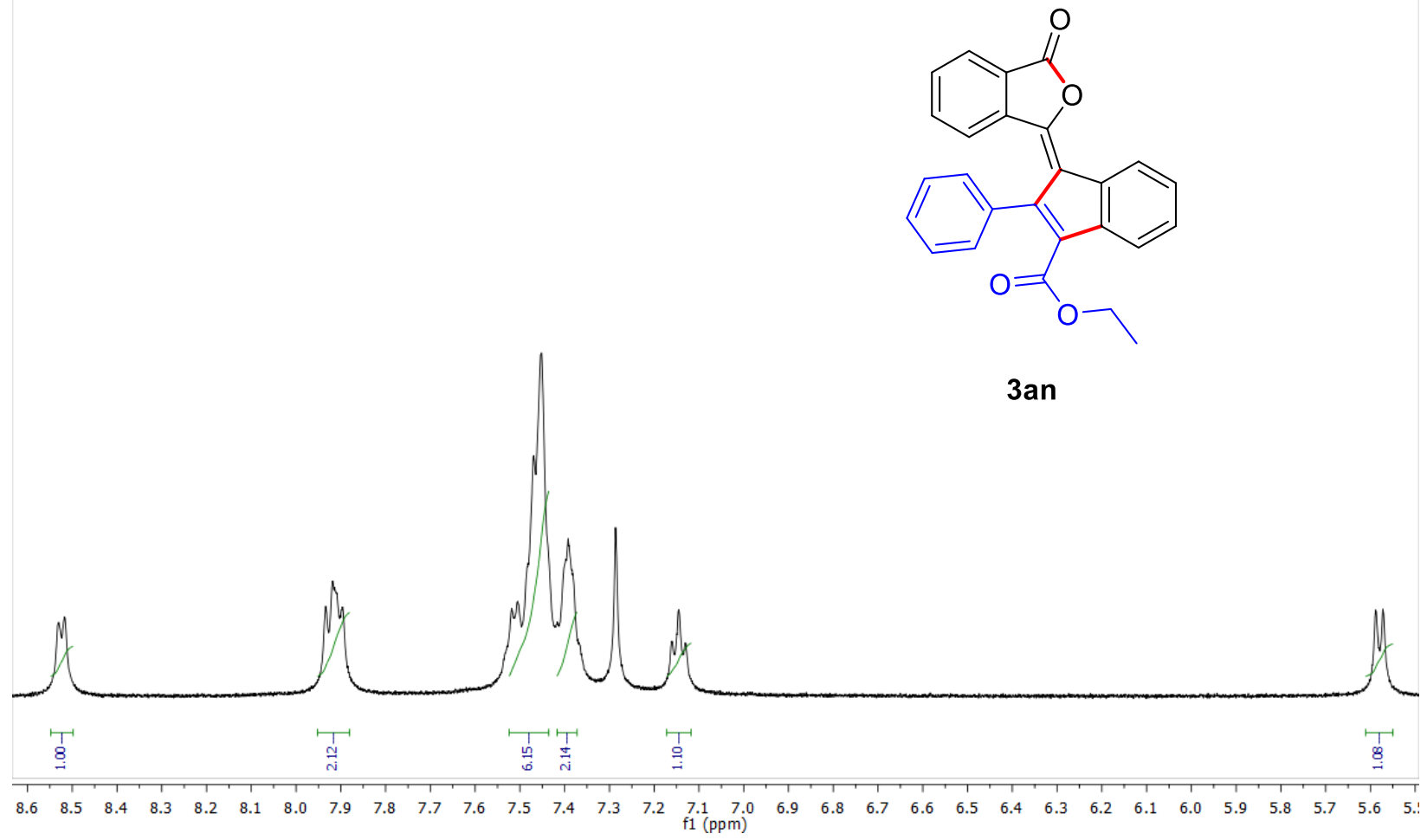



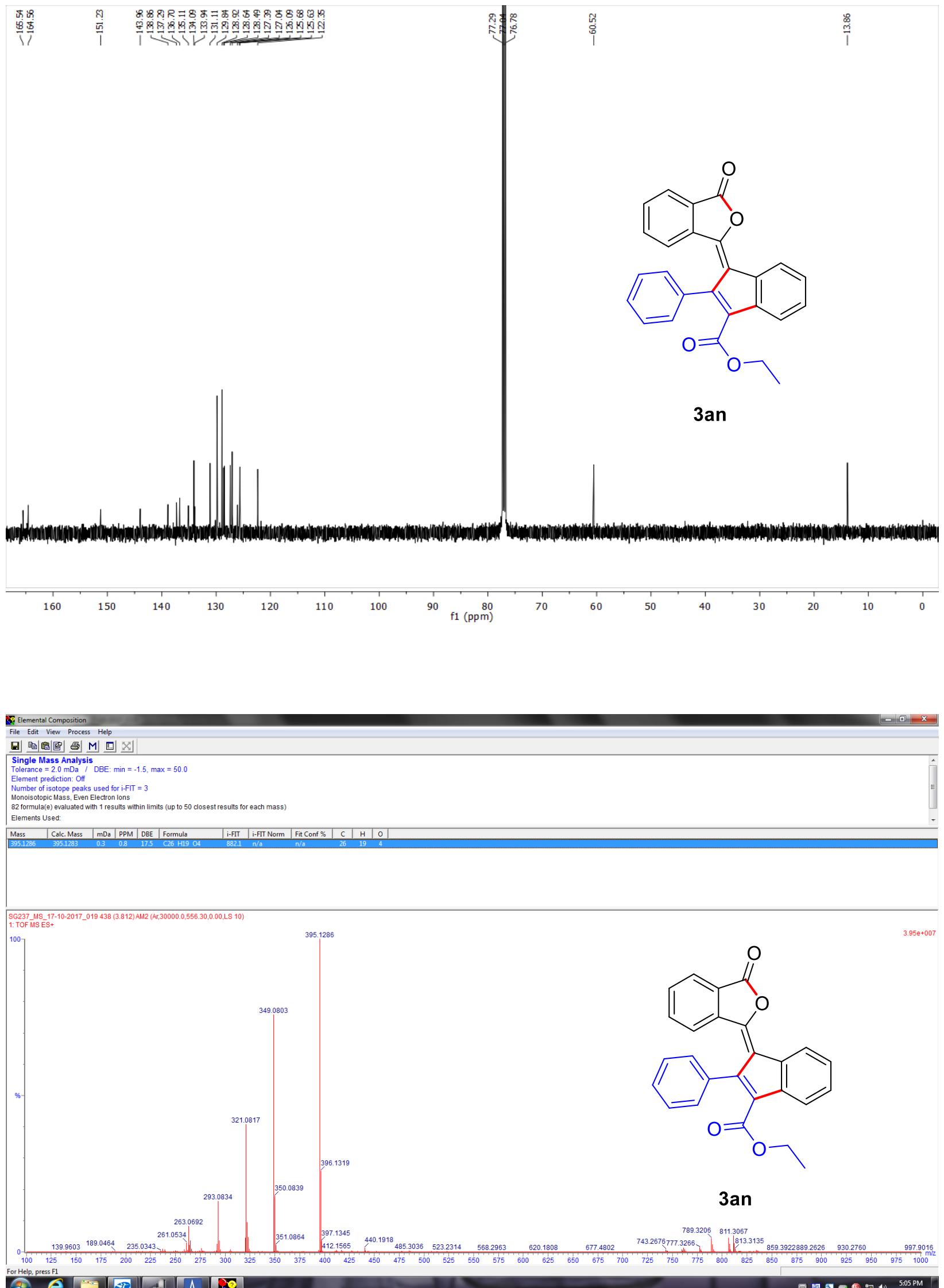


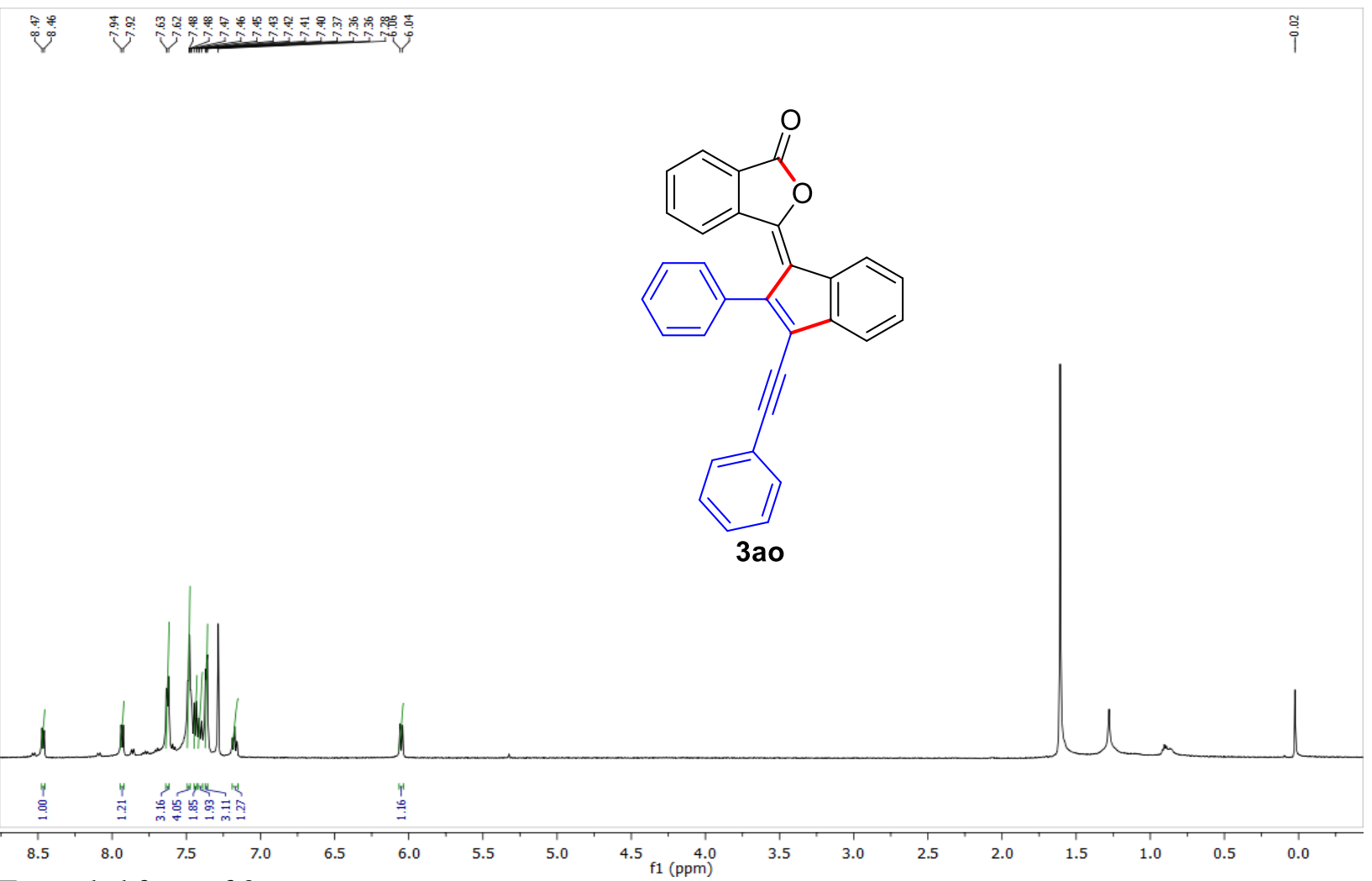

Expanded form of 3ao:

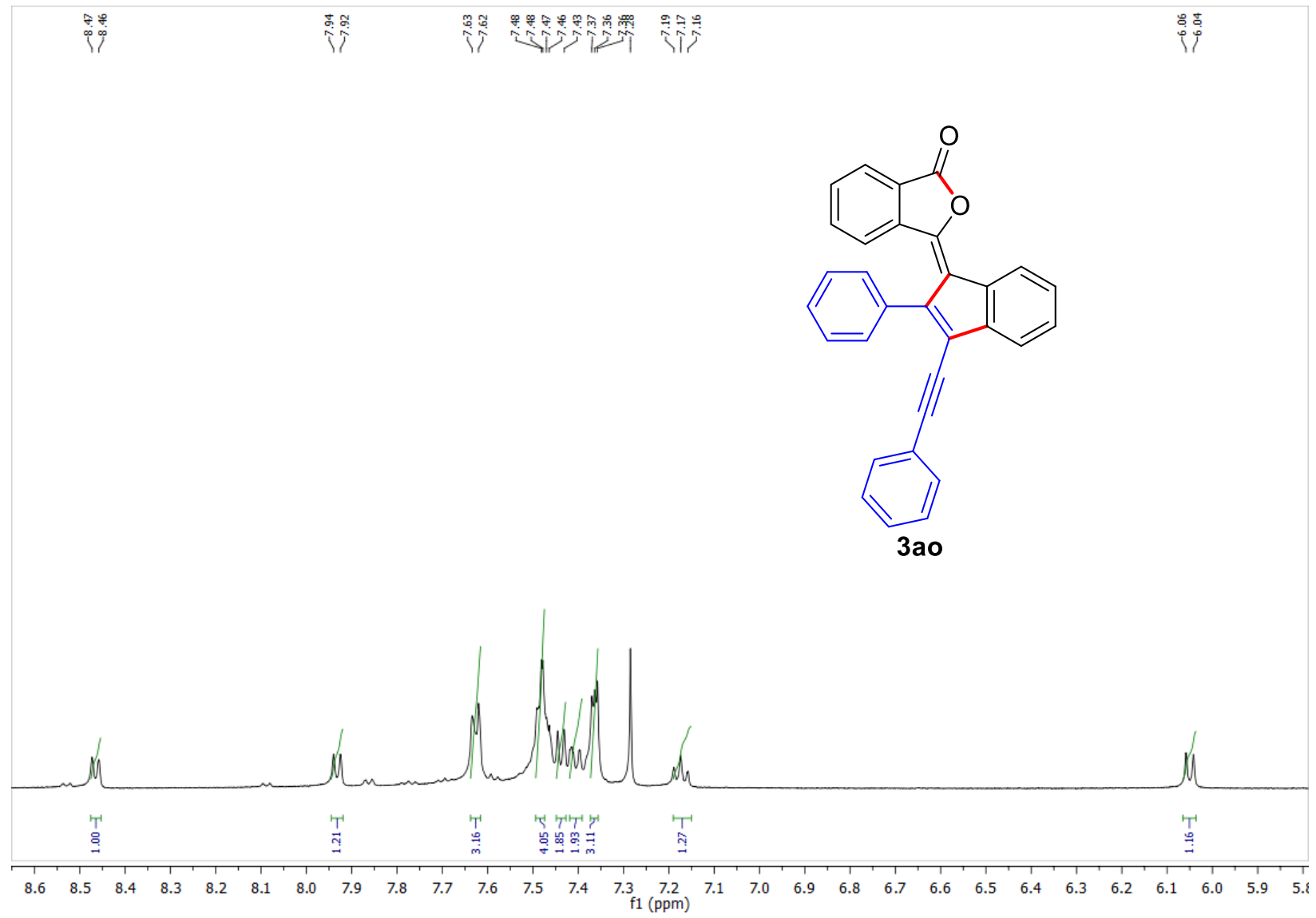



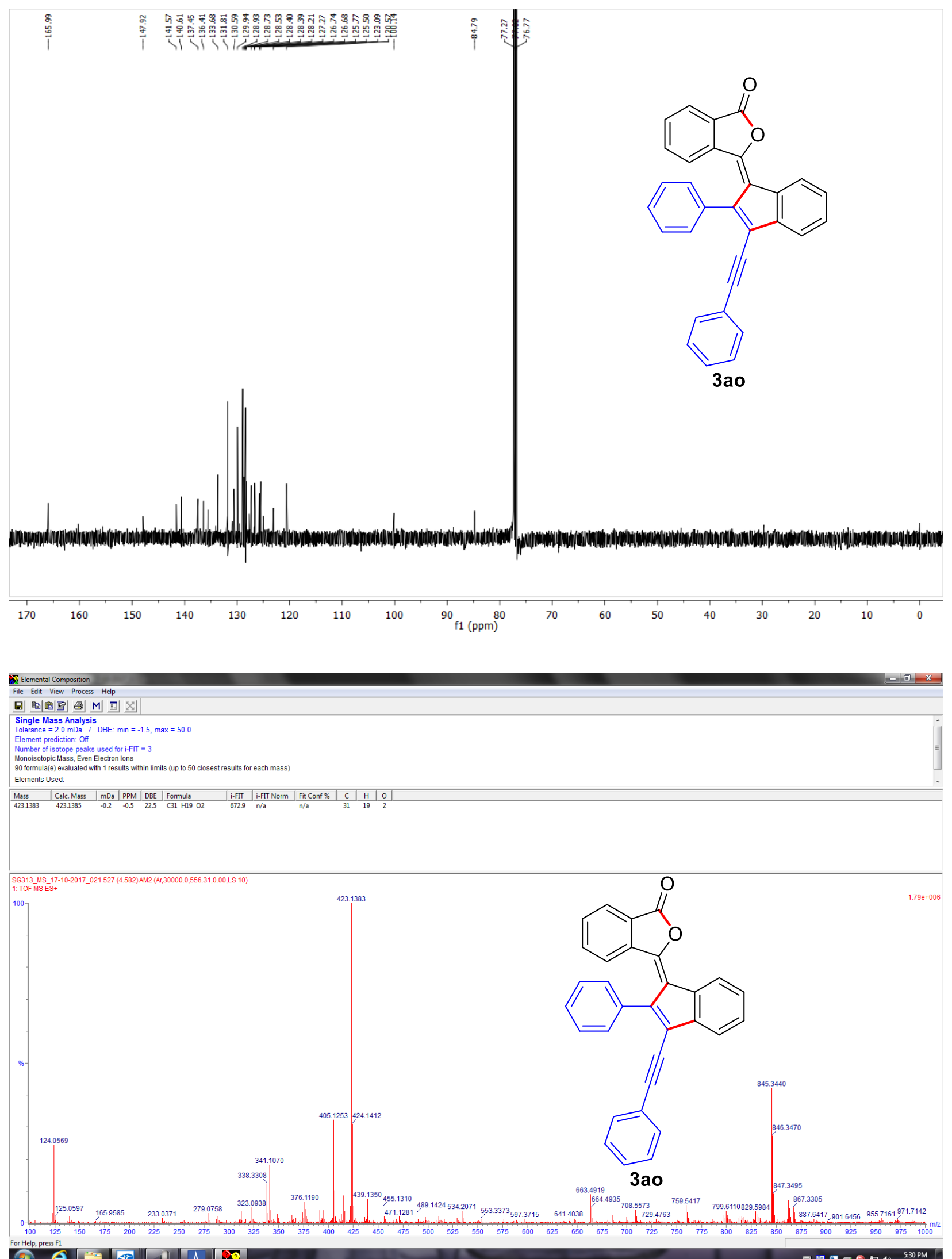


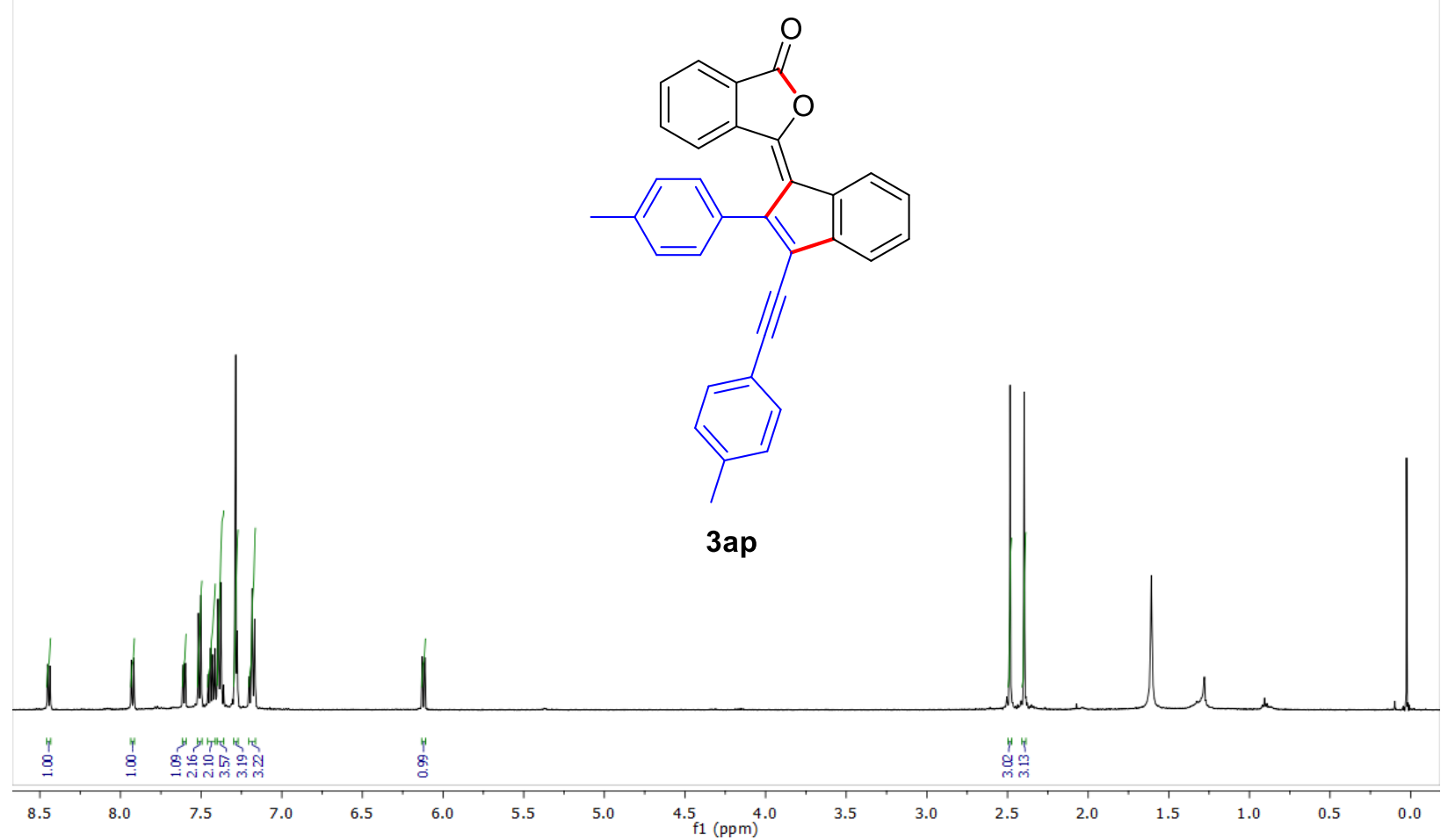

Expanded form of 3ap:

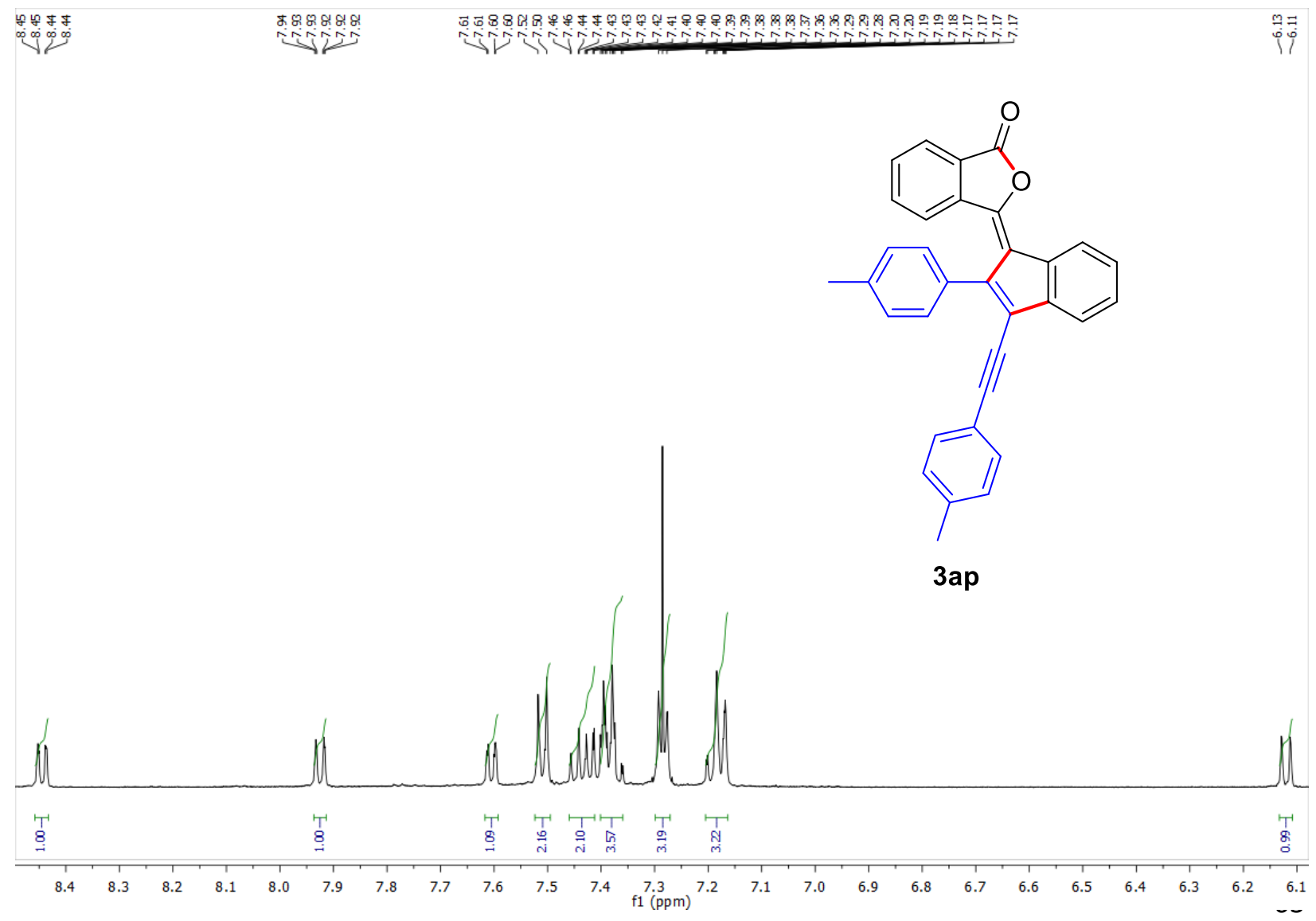



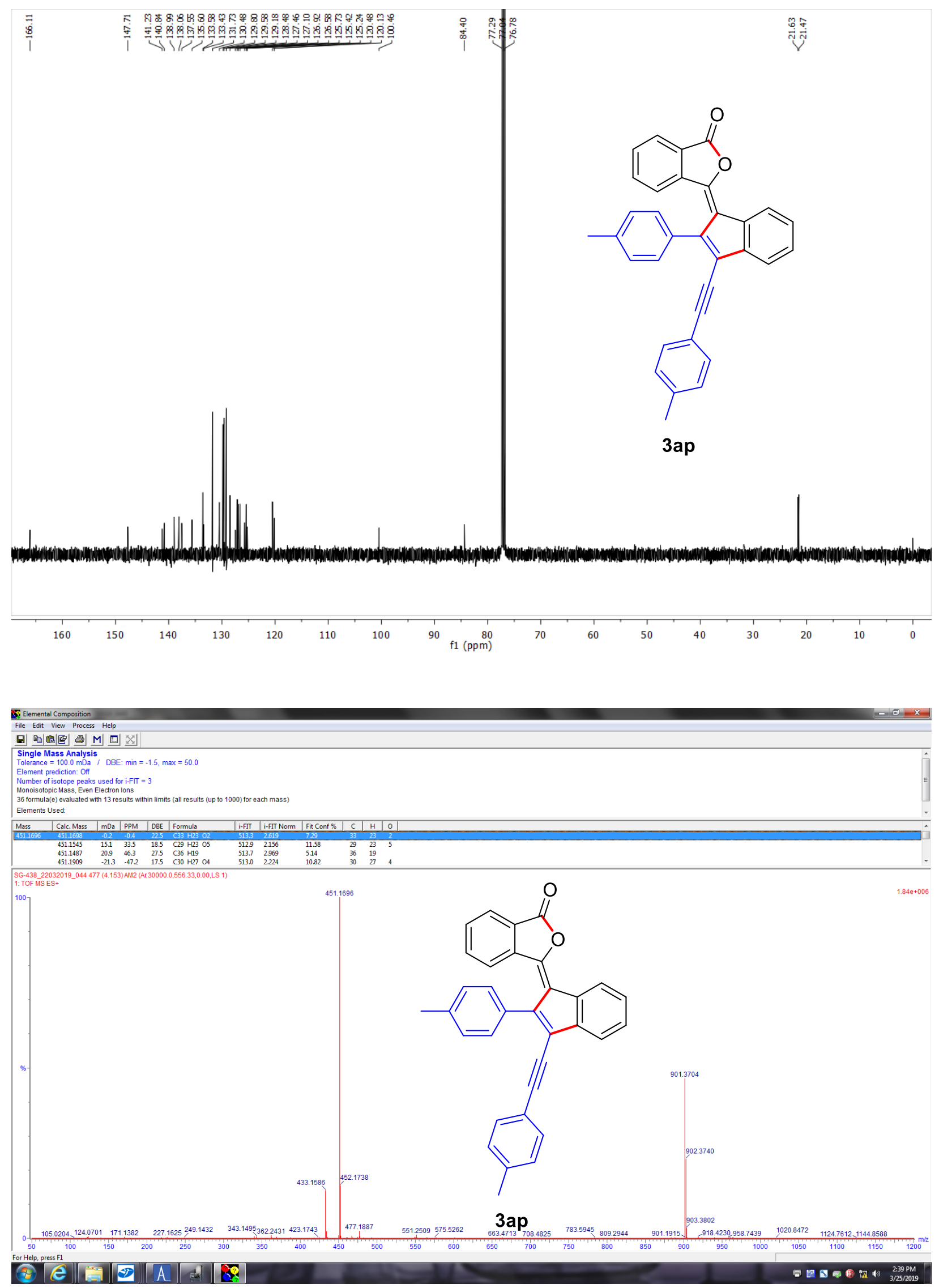


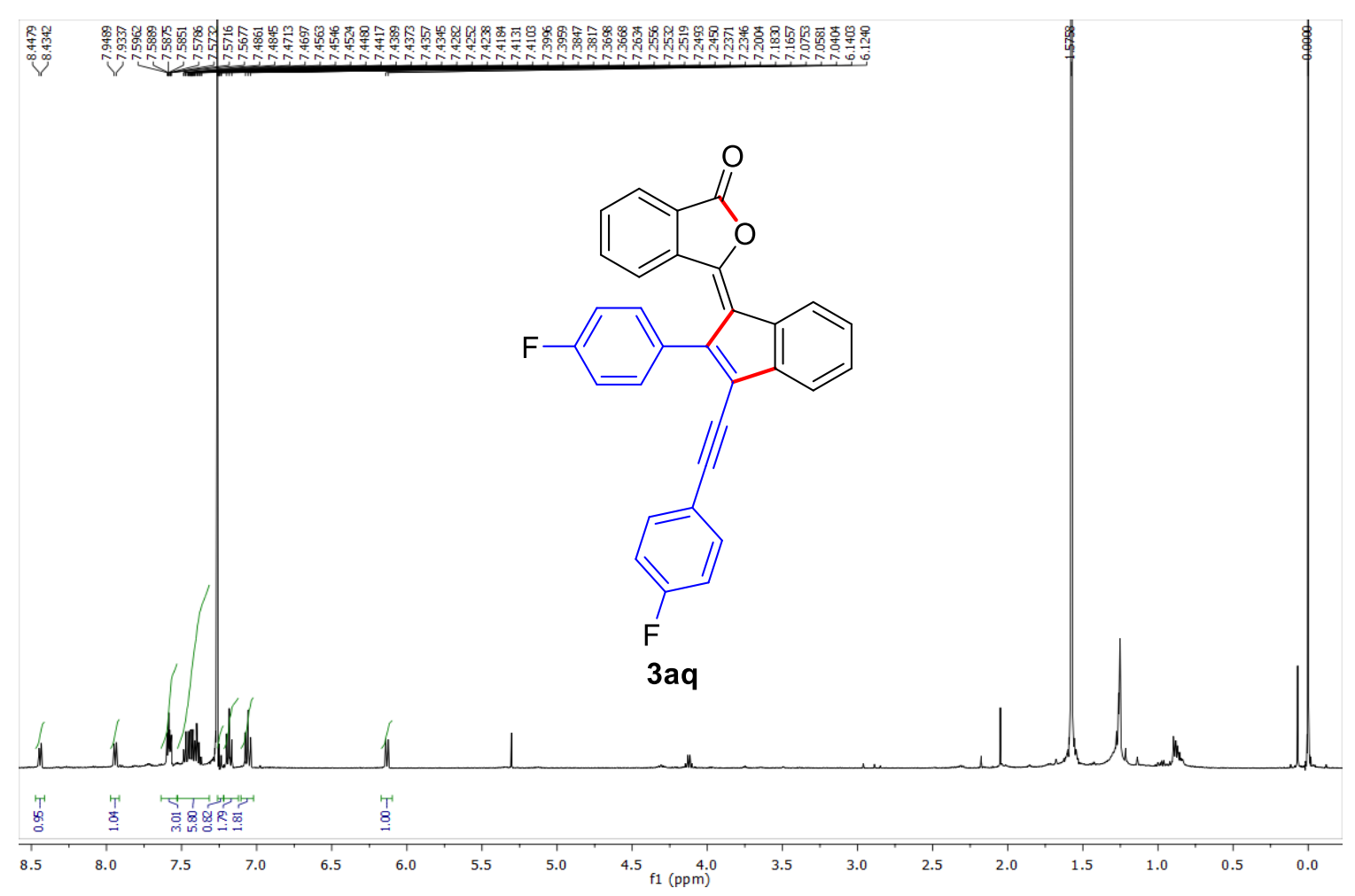

Expanded form of 3aq:

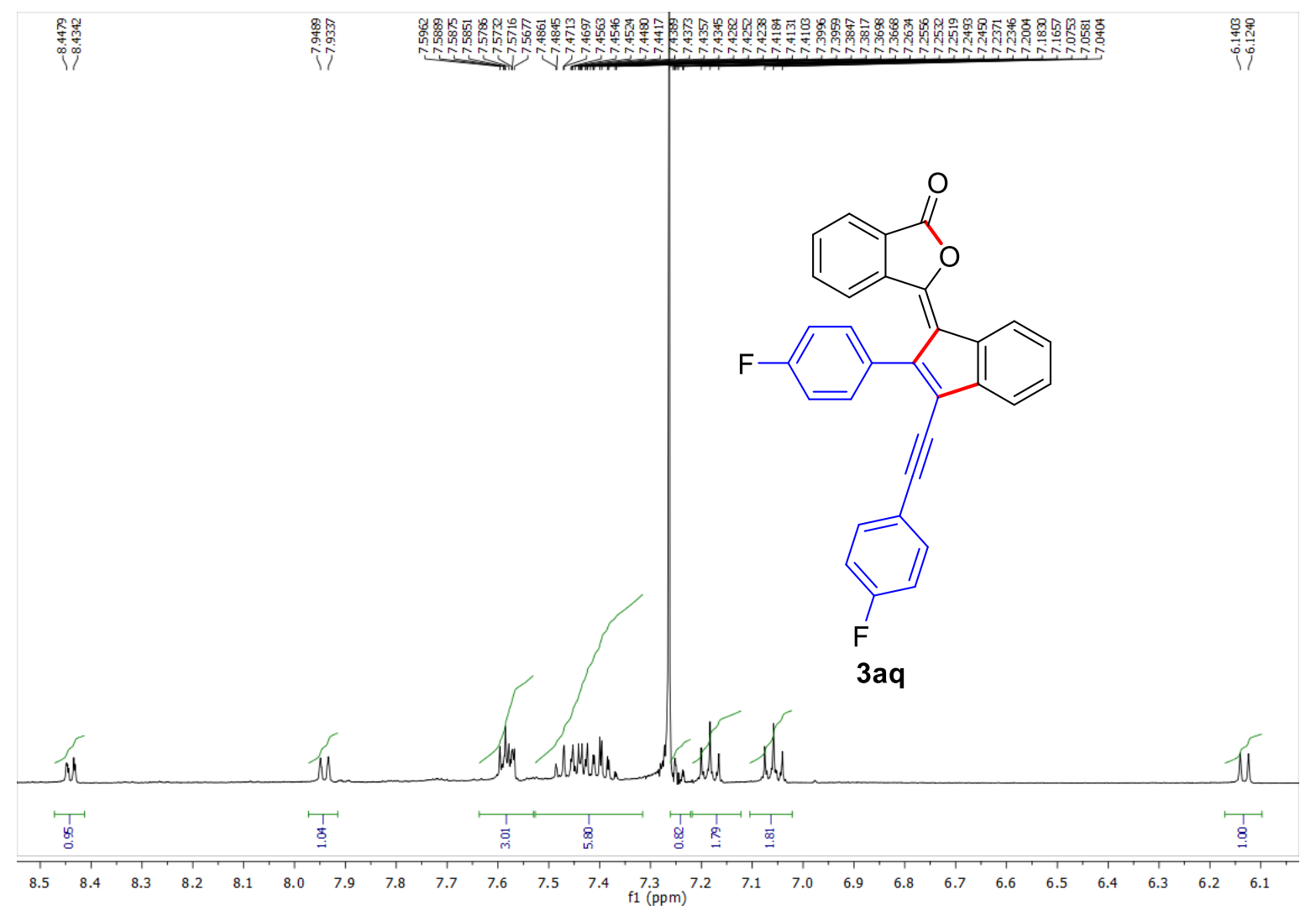



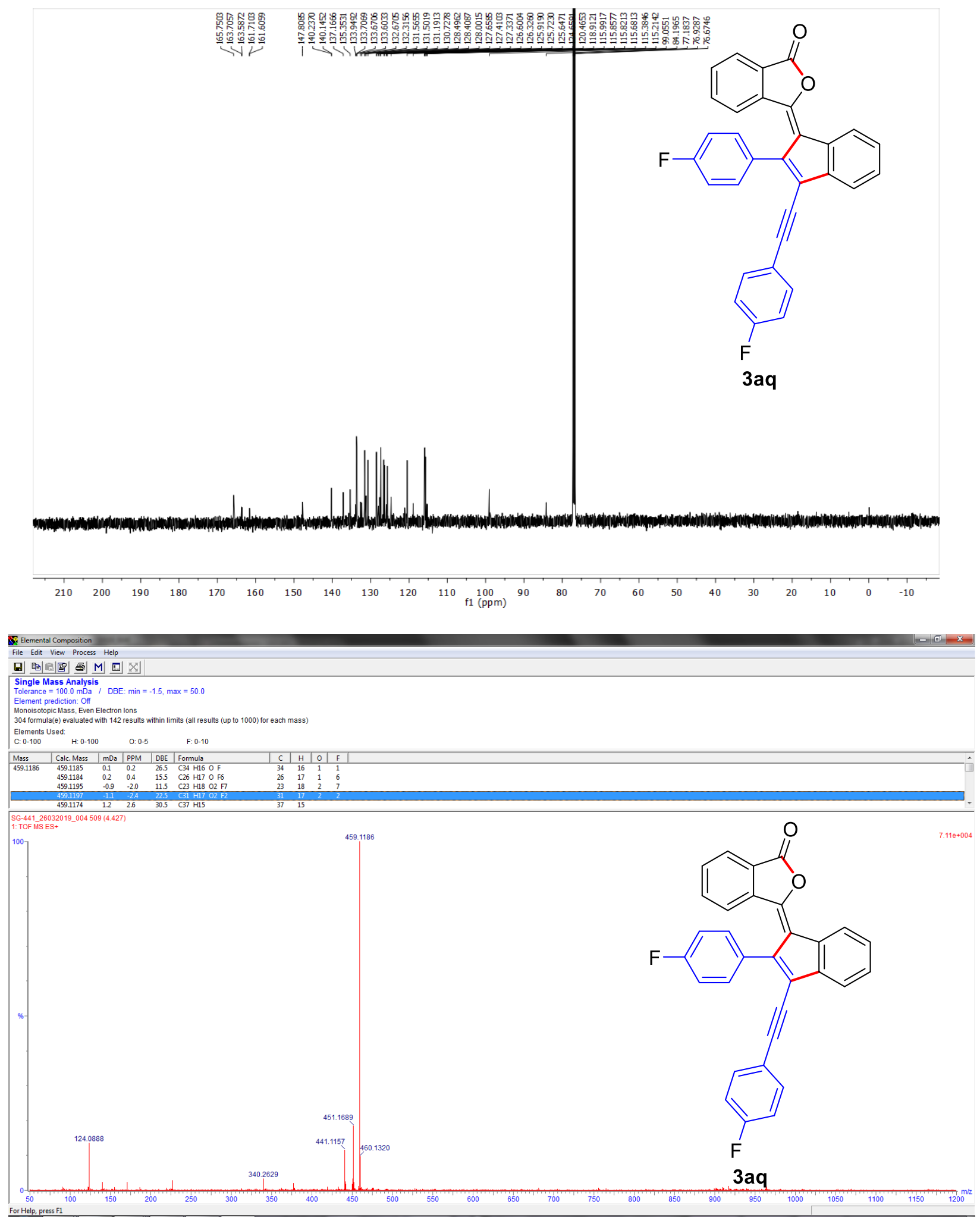


\section{Competitive Experiment:}

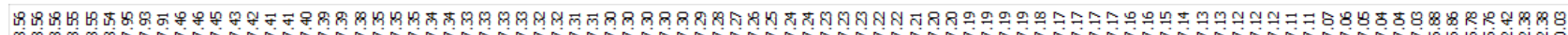

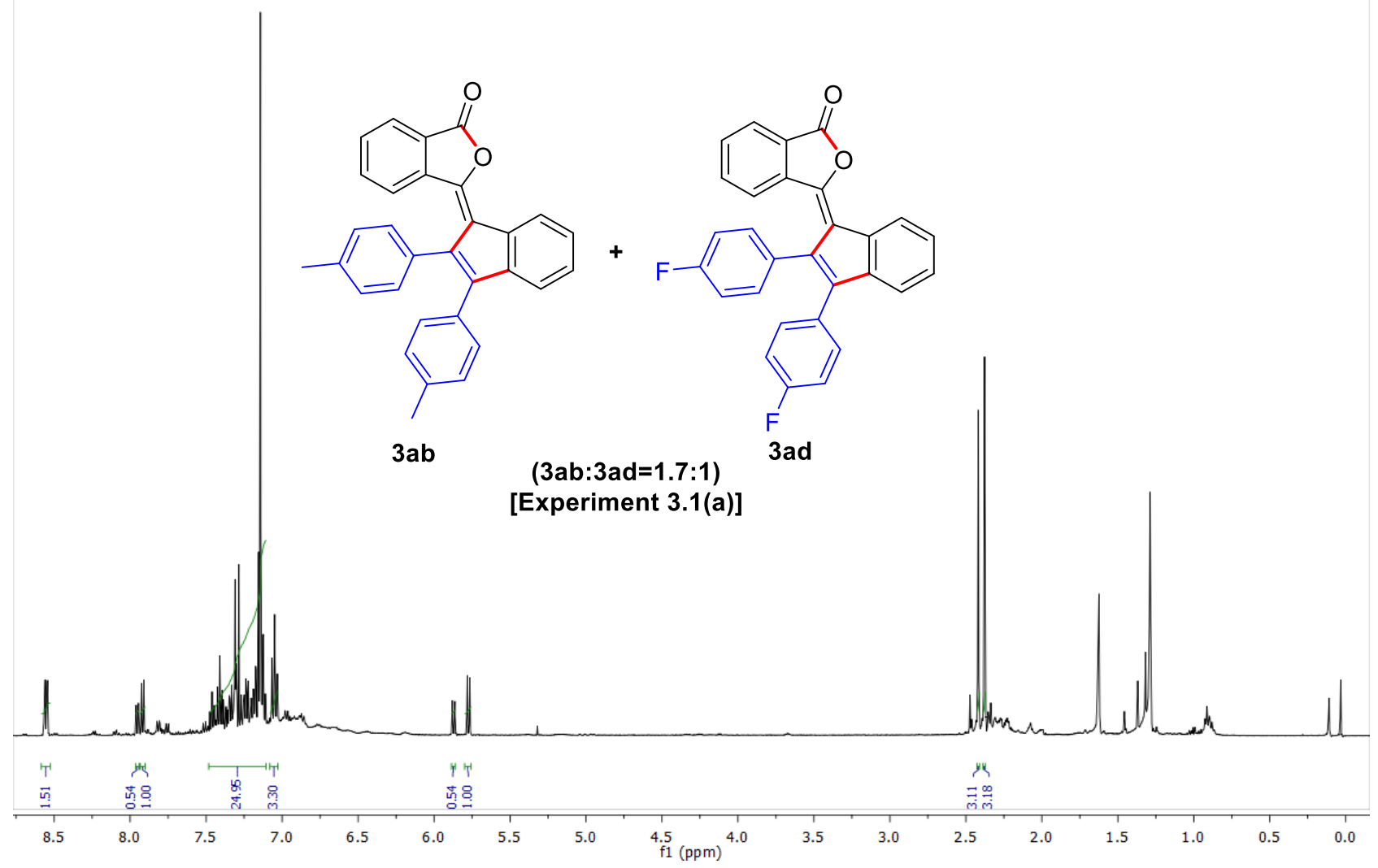


ғ क

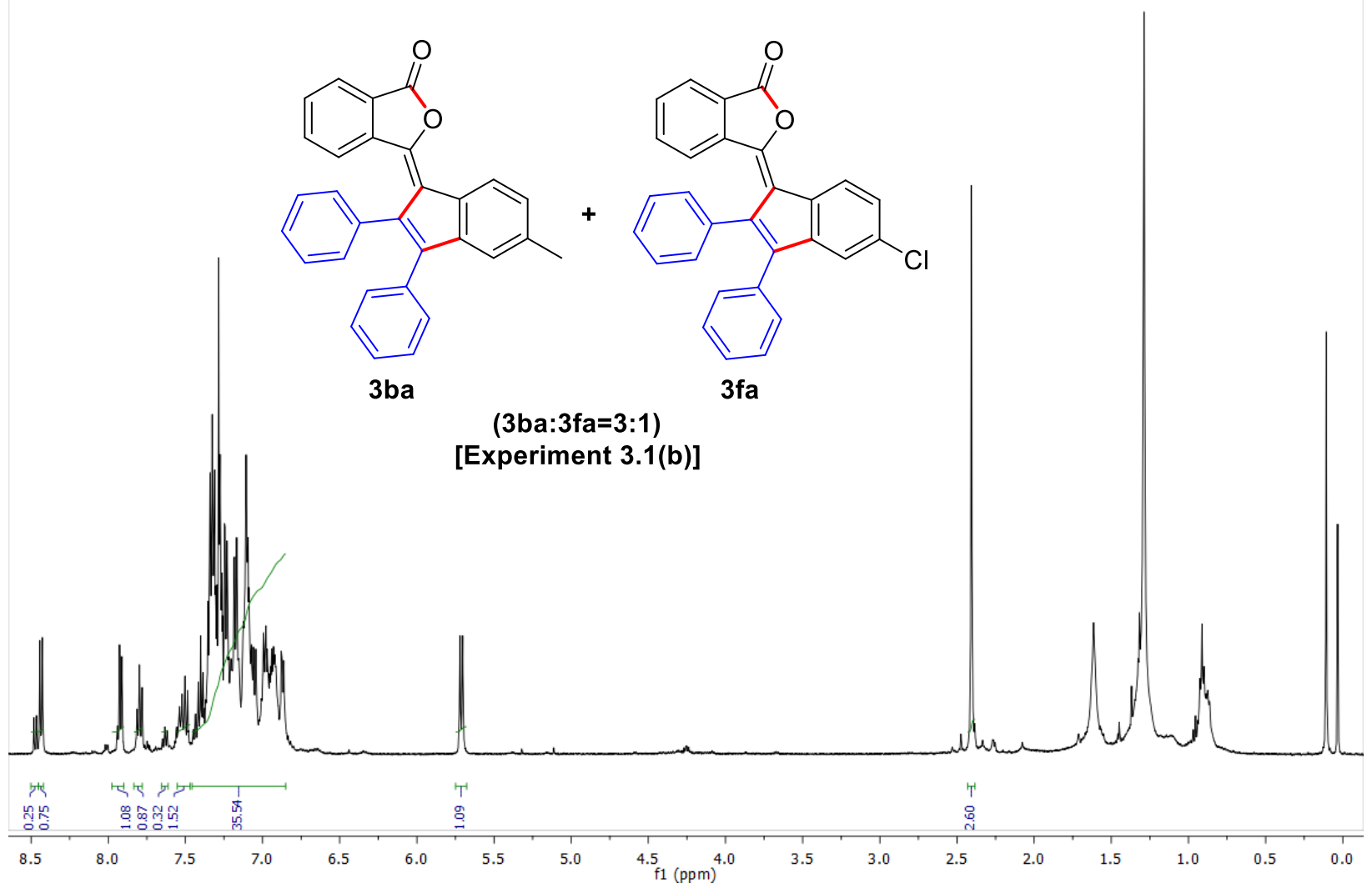


Rin

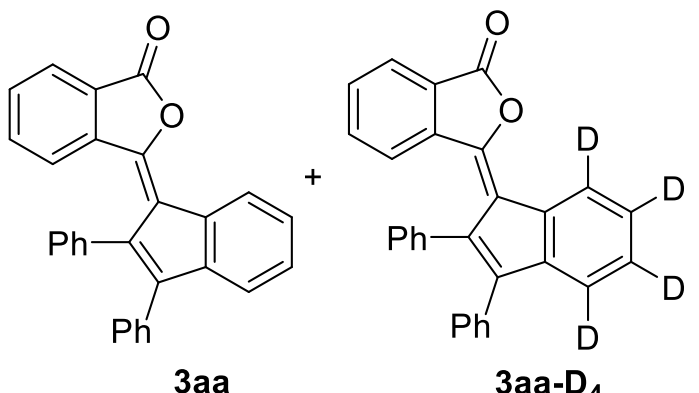

[Experiment 3.2(c)]

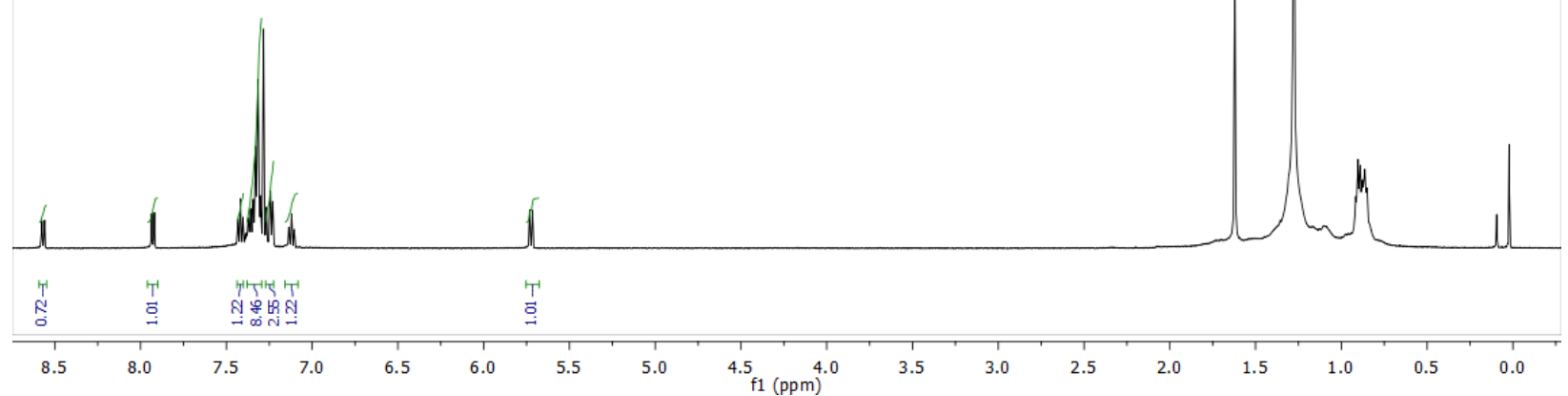




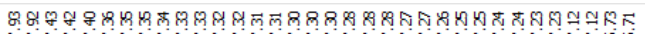

:
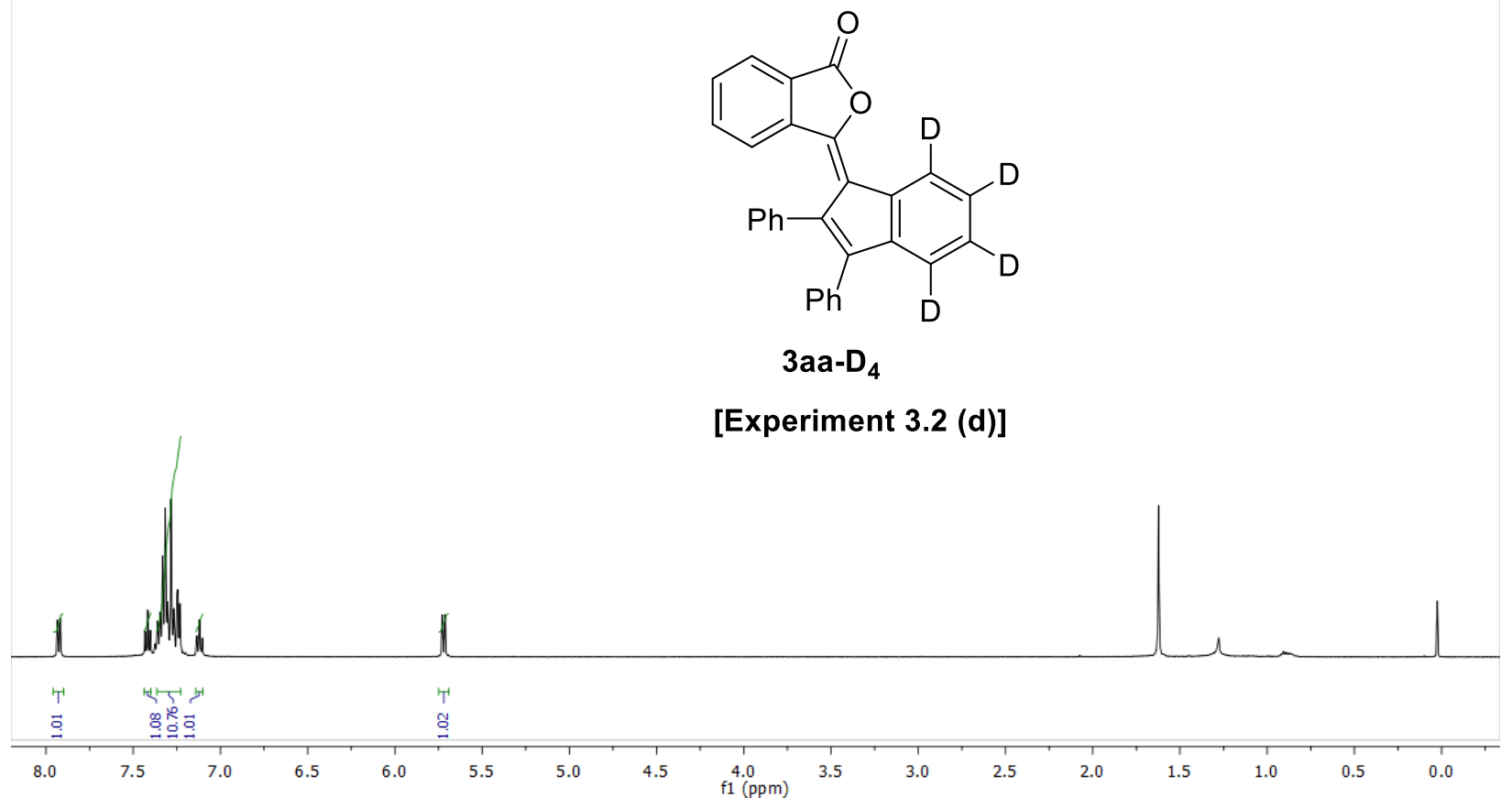

70 


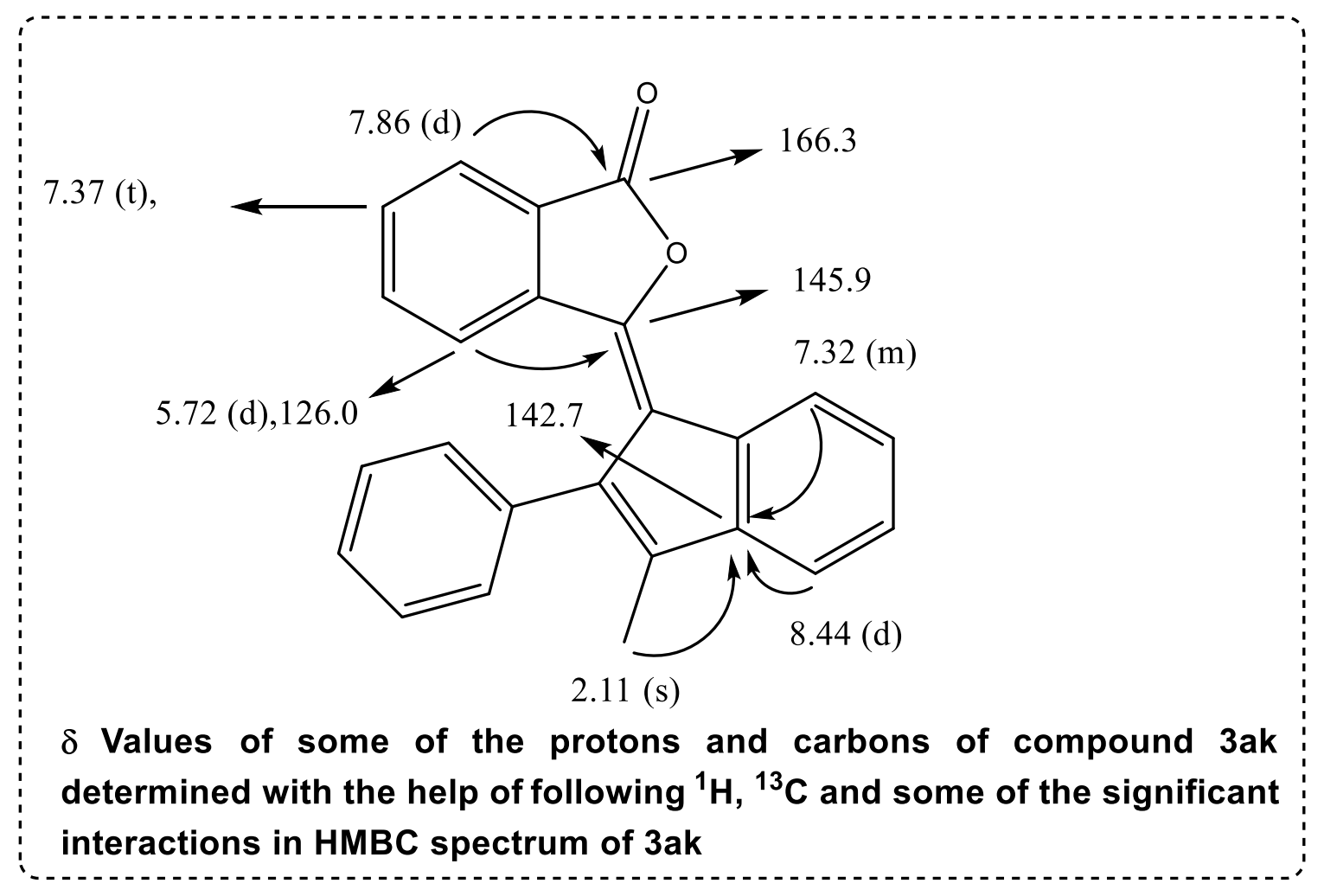


HMQC:

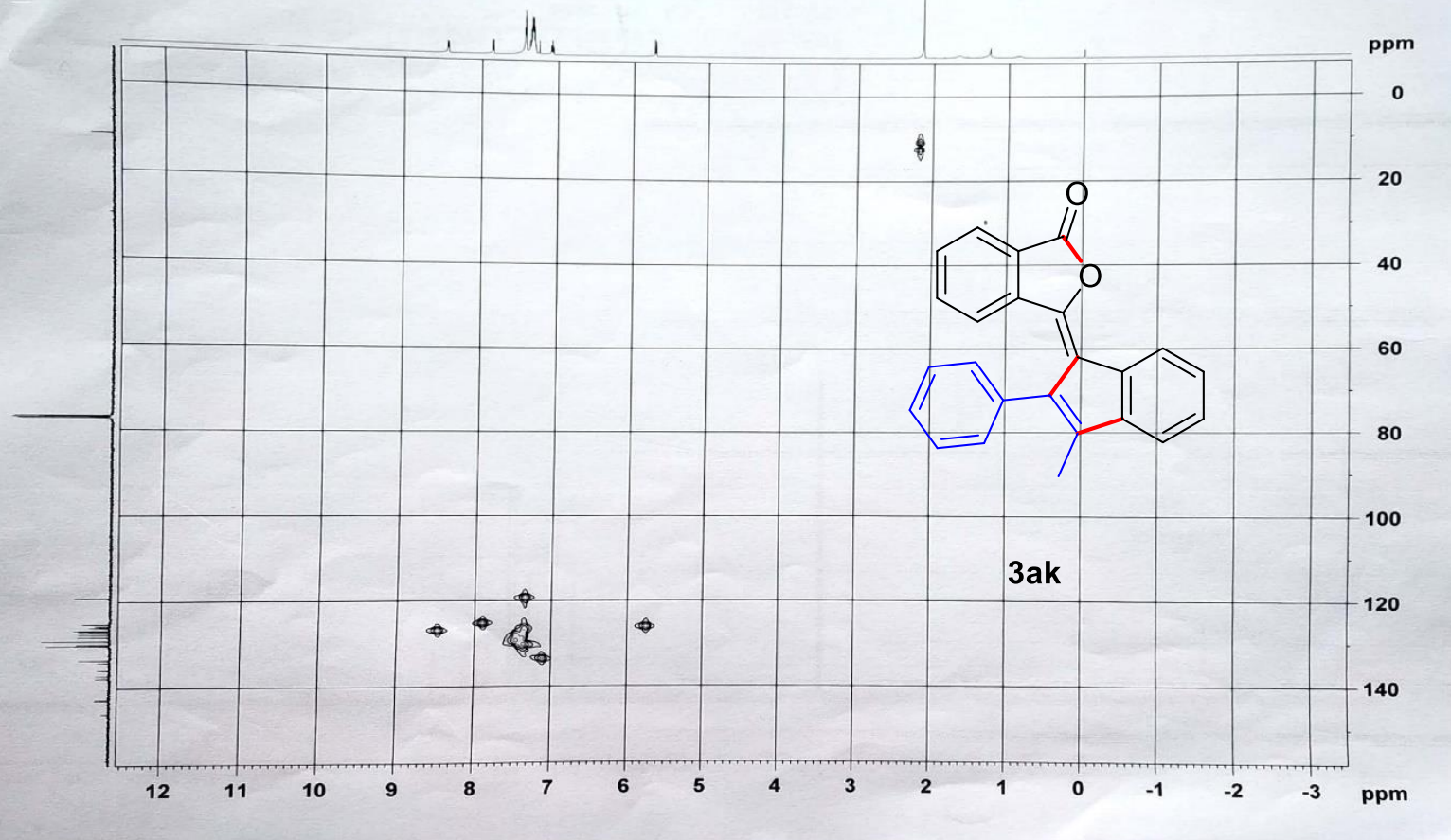

27362
36139

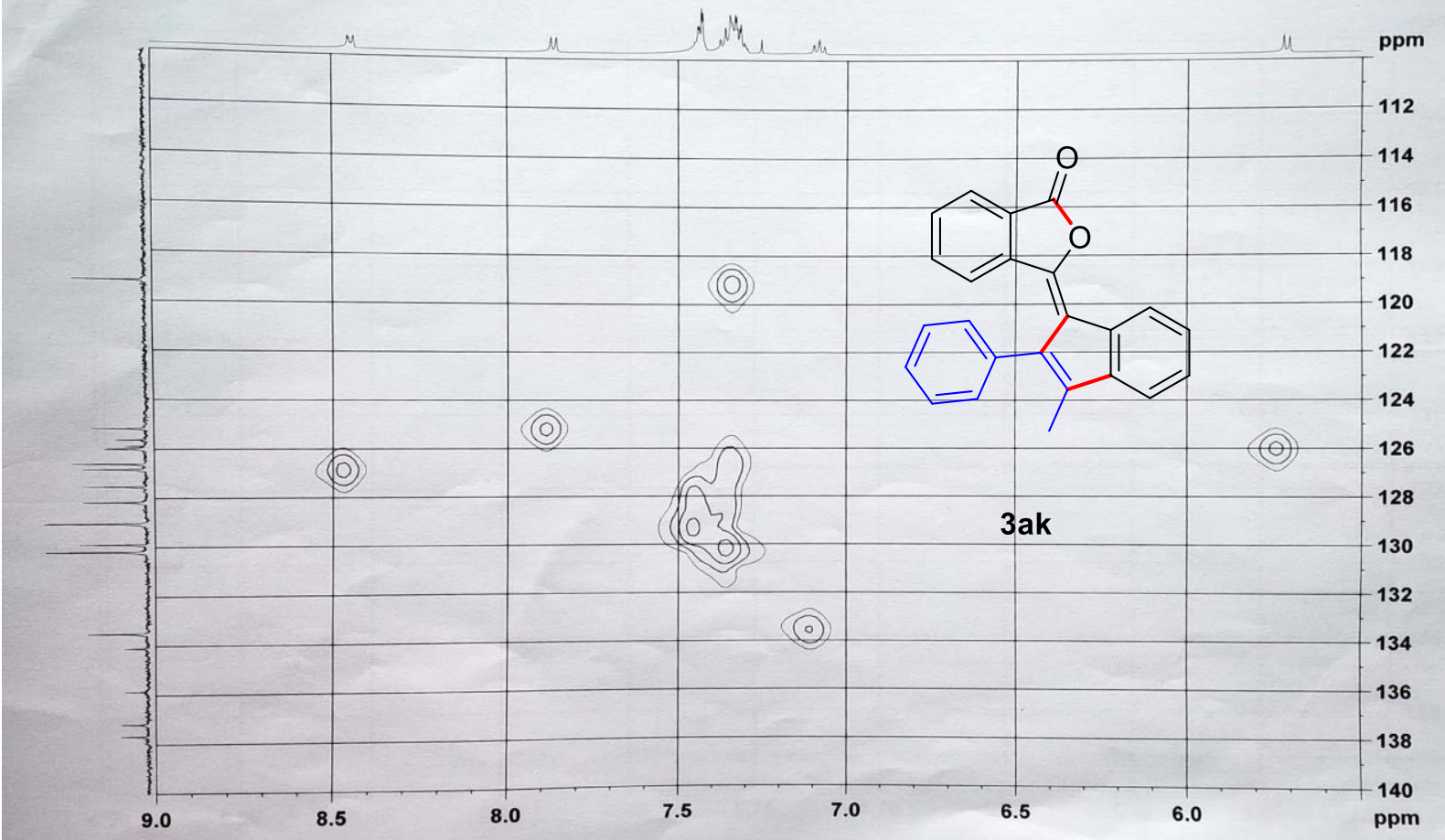


HMBC:

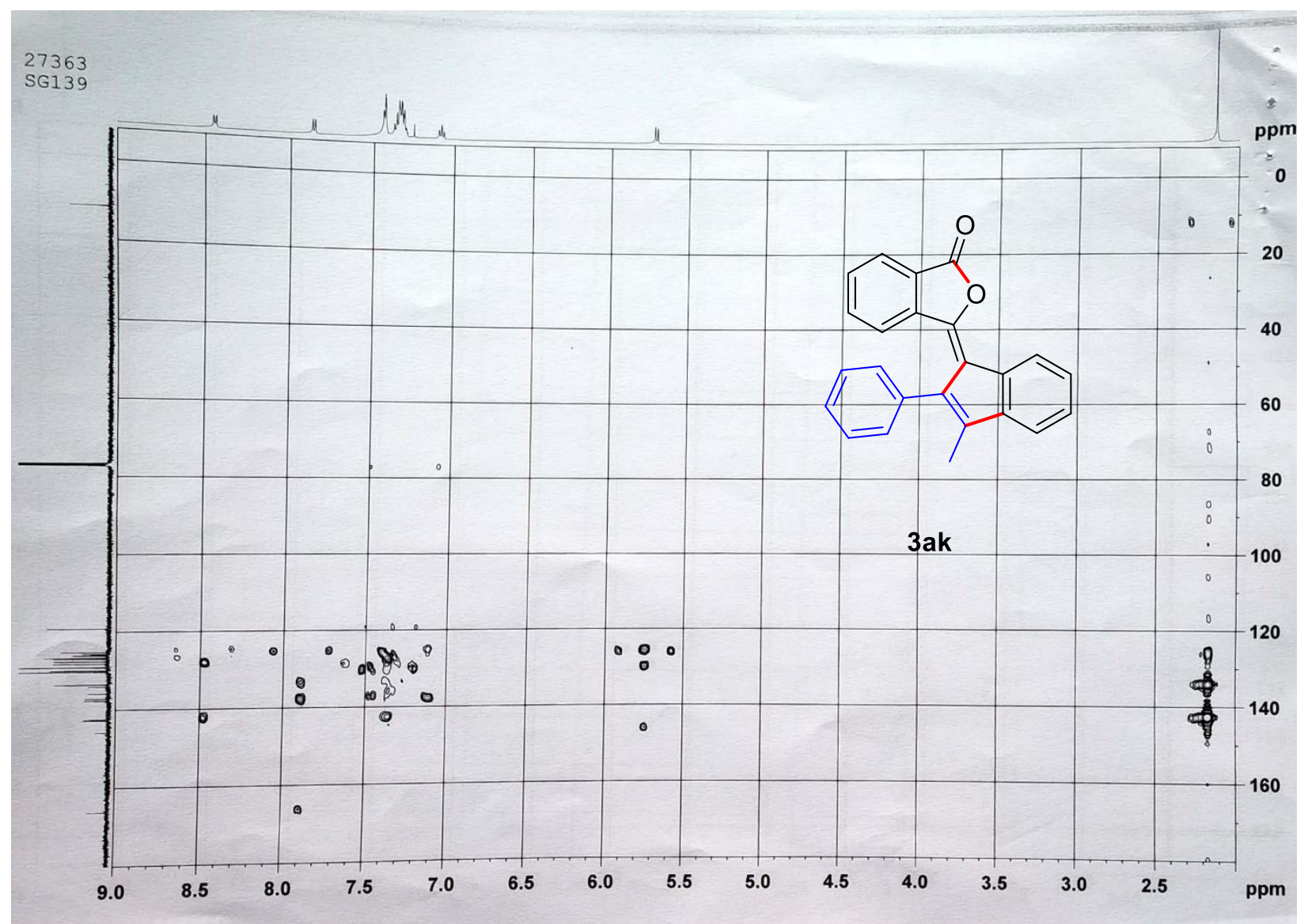


<smiles>O=C1C(O)=C(c2cccs2)C(=O)c2ccccc21</smiles>
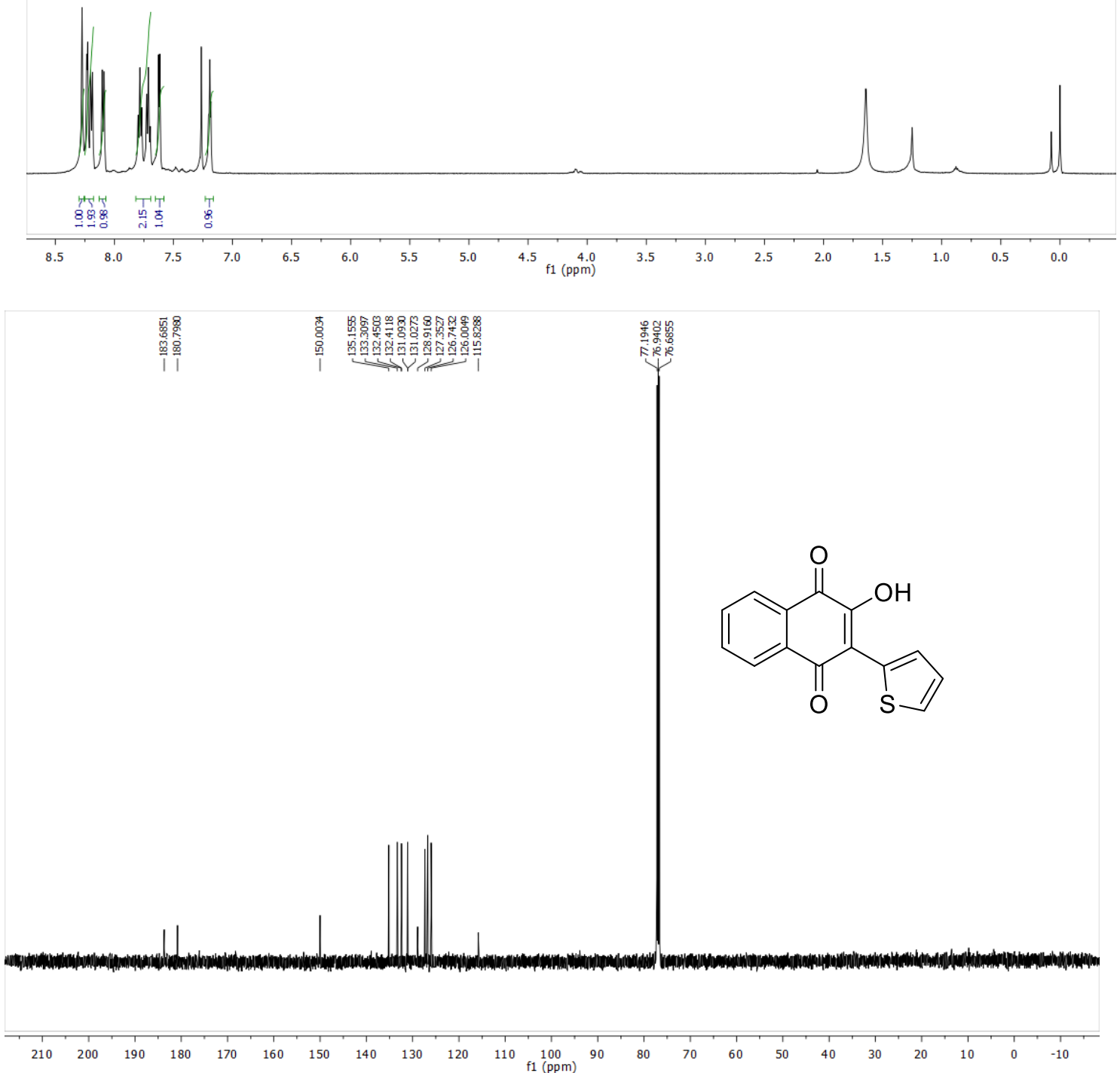

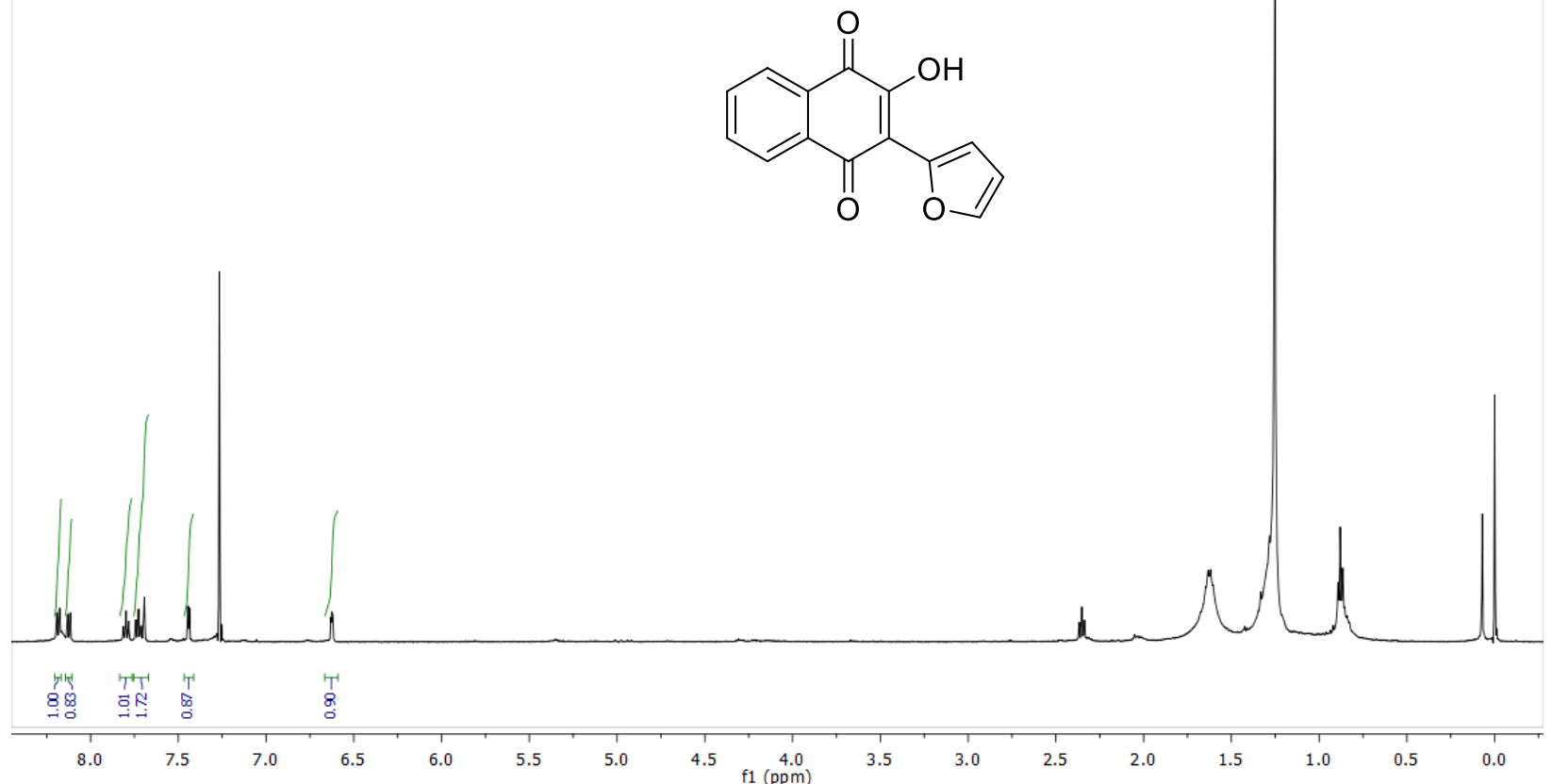